\title{
First stereospecific synthesis of $(E)$ or $(Z) \alpha$-fluoroenones via a kinetically controlled Negishi coupling reaction
}

Guillaume Dutheuil, Clotilde Paturel, Xinsheng Lei, Samuel Couve-Bonnaire and Xavier

Pannecoucke*

IRCOF-ECOFH, UMR CNRS 6014, INSA de ROUEN, rue Tesnière, 76131 Mont-Saint-Aignan, France.

Fax: (33) 2.35.52.29.59; Tel: (33) 2.35.52.24.27; E-mail: xavier.pannecoucke@insa-rouen.fr

\section{Table of contents:}

General experimental information

Experimental procedure for the obtention of $4 a(Z)$ from $E / Z$ mixture of bromofluoroolefin 2

${ }^{1} \mathrm{H},{ }^{13} \mathrm{C}$ and ${ }^{19} \mathrm{~F}$ NMR spectra of compound $\mathbf{2}_{\mathbf{1}}(\mathbf{Z})$

Characterization of compound $4 \mathrm{a}_{1}(Z) ;{ }^{1} \mathrm{H},{ }^{13} \mathrm{C}$ and ${ }^{19} \mathrm{~F}$ NMR spectra

${ }^{1} \mathrm{H},{ }^{13} \mathrm{C}$ and ${ }^{19} \mathrm{~F}$ NMR spectra of compound $\mathbf{2}_{\mathbf{2}}(\boldsymbol{Z})$

Characterization of compound $\mathbf{4 a}_{\mathbf{2}}(\boldsymbol{Z}) ;{ }^{1} \mathrm{H},{ }^{13} \mathrm{C}$ and ${ }^{19} \mathrm{~F}$ NMR spectra ${ }^{1} \mathrm{H},{ }^{13} \mathrm{C}$ and ${ }^{19} \mathrm{~F}$ NMR spectra of compound $\mathbf{2}_{\mathbf{3}}(\boldsymbol{Z})$ ${ }^{1} \mathrm{H},{ }^{13} \mathrm{C}$ and ${ }^{19} \mathrm{~F}$ NMR spectra of compound $\mathbf{2}_{5}(\mathbf{Z})$ ${ }^{1} \mathrm{H},{ }^{13} \mathrm{C}$ and ${ }^{19} \mathrm{~F}$ NMR spectra of compound $\mathbf{2}_{6}(\boldsymbol{Z})$ 
${ }^{1} \mathrm{H},{ }^{13} \mathrm{C}$ and ${ }^{19} \mathrm{~F}$ NMR spectra of compound $\mathbf{2}_{7}(\mathbf{Z})$

S19 Characterization of compound $4 \mathrm{a}_{\mathbf{7}}(\mathbf{Z}) ;{ }^{1} \mathrm{H},{ }^{13} \mathrm{C}$ and ${ }^{19} \mathrm{~F}$ NMR spectra

${ }^{1} \mathrm{H},{ }^{13} \mathrm{C}$ and ${ }^{19} \mathrm{~F}$ NMR spectra of compound $\mathbf{2}_{8}(Z)$

Characterization of compound $4 \mathbf{a}_{8}(Z) ;{ }^{1} \mathrm{H},{ }^{13} \mathrm{C}$ and ${ }^{19} \mathrm{~F}$ NMR spectra

Experimental procedure for the obtention of $\mathbf{4} \mathbf{b}_{1}(Z)$ from $E / Z$ mixture of bromofluoroolefin 2

Characterization of compound $\mathbf{4} \mathbf{b}_{1}(Z) ;{ }^{1} \mathrm{H},{ }^{13} \mathrm{C}$ and ${ }^{19} \mathrm{~F}$ NMR spectra

$\mathrm{S} 24$

Experimental procedure for the obtention of $\mathbf{4 b}_{\mathbf{2}}(\boldsymbol{Z})$ from $E / Z$ mixture of bromofluoroolefin 2

$\mathrm{S} 25$

Characterization of compound $4 \mathbf{b}_{2}(Z) ;{ }^{1} \mathrm{H},{ }^{13} \mathrm{C}$ and ${ }^{19} \mathrm{~F}$ NMR spectra

$\mathrm{S} 26$

Experimental procedure for the obtention of $4 \mathrm{c}(Z)$ from $E / Z$ mixture of bromofluoroolefin 2

$\mathrm{S} 27$

$\mathrm{S} 28$

Characterization of compound $4 \mathbf{c}_{1}(Z) ;{ }^{1} \mathrm{H},{ }^{13} \mathrm{C}$ and ${ }^{19} \mathrm{~F}$ NMR spectra

$\mathrm{S} 29$

Characterization of compound $\mathbf{4} \mathbf{c}_{2}(Z) ;{ }^{1} \mathrm{H},{ }^{13} \mathrm{C}$ and ${ }^{19} \mathrm{~F}$ NMR spectra

S30

Experimental procedure for the obtention of $7(Z)$ from $E / Z$ mixture of bromofluoroolefin 2

S31

Characterization of compound $\mathbf{7}_{1}(Z) ;{ }^{1} \mathrm{H},{ }^{13} \mathrm{C}$ and ${ }^{19} \mathrm{~F}$ NMR spectra

S32

Characterization of compound $\mathbf{7}_{2}(Z) ;{ }^{1} \mathrm{H},{ }^{13} \mathrm{C}$ and ${ }^{19} \mathrm{~F}$ NMR spectra

S33

Experimental procedure for the obtention of $\mathbf{4 d}$

S34

Characterization of compound $\mathbf{4} \mathbf{d}_{1}(\boldsymbol{Z}) ;{ }^{1} \mathrm{H},{ }^{13} \mathrm{C}$ and ${ }^{19} \mathrm{~F}$ NMR spectra

S35

Experimental procedure for the obtention of $\mathbf{4 a}(E)$ from bromofluoroolefin 2(Z)

S36

Characterization of compound $4 \mathrm{a}_{1}(E) ;{ }^{1} \mathrm{H},{ }^{13} \mathrm{C}$ and ${ }^{19} \mathrm{~F}$ NMR spectra

S37

Characterization of compound $4 \mathbf{a}_{2}(E) ;{ }^{1} \mathrm{H},{ }^{13} \mathrm{C}$ and ${ }^{19} \mathrm{~F}$ NMR spectra

S38

Characterization of compound $4 \mathbf{a}_{3}(E) ;{ }^{1} \mathrm{H},{ }^{13} \mathrm{C}$ and ${ }^{19} \mathrm{~F}$ NMR spectra

S39

Characterization of compound $4 \mathrm{a}_{4}(E) ;{ }^{1} \mathrm{H},{ }^{13} \mathrm{C}$ and ${ }^{19} \mathrm{~F}$ NMR spectra

$\mathrm{S} 40$

Characterization of compound $\mathbf{4 a}_{5}(E) ;{ }^{1} \mathrm{H},{ }^{13} \mathrm{C}$ and ${ }^{19} \mathrm{~F}$ NMR spectra

S41

Characterization of compound $4 \mathbf{a}_{\mathbf{7}}(\boldsymbol{E}) ;{ }^{1} \mathrm{H},{ }^{13} \mathrm{C}$ and ${ }^{19} \mathrm{~F}$ NMR spectra

Characterization of compound $4 \mathbf{a}_{8}(E) ;{ }^{1} \mathrm{H},{ }^{13} \mathrm{C}$ and ${ }^{19} \mathrm{~F}$ NMR spectra 
Characterization of compound $\mathbf{4 C}_{1}(E) ;{ }^{1} \mathrm{H},{ }^{13} \mathrm{C}$ and ${ }^{19} \mathrm{~F}$ NMR spectra

Characterization of compound $7_{1}(E) ;{ }^{1} \mathrm{H},{ }^{13} \mathrm{C}$ and ${ }^{19} \mathrm{~F}$ NMR spectra 


\section{First stereospecific synthesis of $(E)$ or $(Z) \alpha$-fluoroenones via a kinetically controlled Negishi coupling reaction \\ Guillaume Dutheuil, Clotilde Paturel, Xinsheng Lei, Samuel Couve-Bonnaire and Xavier \\ Pannecoucke*}

IRCOF-ECOFH, UMR CNRS 6014, INSA de ROUEN, rue Tesnière, 76131 Mont-Saint-Aignan, France.

Fax: (33) 2.35.52.29.59; Tel: (33) 2.35.52.24.27; E-mail: xavier.pannecoucke@insa-rouen.fr

\section{Experimental Section.}

General: ${ }^{19} \mathrm{~F} 4(282.5 \mathrm{MHz}),{ }^{13} \mathrm{C}$ NMR $(75.5 \mathrm{MHz})$ and ${ }^{1} \mathrm{H}$ NMR (300 MHz) spectra were recorded on a $300 \mathrm{MHz}$ spectrometer. All samples were taken in $\mathrm{CDCl}_{3}$ solvent unless noted otherwise. All chemical shifts are reported in parts per million downfield (positive) of the standard : TMS for ${ }^{1} \mathrm{H}$ and ${ }^{13} \mathrm{C}, \mathrm{CFCl}_{3}$ for ${ }^{19} \mathrm{~F}$ NMR. Coupling constants ( $\mathrm{J}$ ) are given in cycles per second (Hz). ${ }^{1} \mathrm{H}$ - and ${ }^{13} \mathrm{C}-\mathrm{NMR}$ peaks were assigned using standard COSY, HMQC and HMBC experiments. All reactions were carried out in oven-dried glassware that was placed under vacuum while hot and cooled under argon. Column Chromatographies were performed on silica gel (70-230 mesh). THF was dried by refluxing and distilling under argon from sodium benzophenone dianion. All reagents were obtained from commercial sources, or prepared from the literature. The bromofluorolefins were prepared by our method. ${ }^{\text {a }}$ 
Representative procedure for the obtention of $(Z)$ fluorinated methylketones $4 \mathbf{a}(Z)$ from an $E / Z$ mixture of bromofluoroolefins 2 . To a mixture of potassium tert-butoxide ( 0.85 eq.), ethylvinylether ( 0.85 eq.) in anhydrous THF ( $5 \mathrm{~mL} / \mathrm{mmol}$ of $t$-BuOK) at $-78^{\circ} \mathrm{C}$ under argon was added dropwise $n$-BuLi in hexanes (1.6M, 0.85 eq.). The mixture was stirred for $30 \mathrm{~min}$ at $-78^{\circ} \mathrm{C}$ and then a solution of dry zincchloride (1.7 eq.) in THF ( $4 \mathrm{~mL} / \mathrm{mmol}^{\text {of }} \mathrm{ZnCl}_{2}$ ) was added dropwise. After $10 \mathrm{~min}$, the cooling bath was removed and the solution was allowed to warm to room temperature for $30 \mathrm{~min}$. The mixture was then added slowly to a solution of palladiumdiacetate (0.025 eq.), triphenylphosphine (0.05 eq.) and mixture of bromofluoroolefins 2 (1 eq.) in anhydrous THF (10 mL/mmol of bromofluoroolefin) at $10^{\circ} \mathrm{C}$ under argon. The mixture was stirred for $1 \mathrm{~h} . \mathrm{HCl}$ aq. (1N) was added and the solution was stirred for $15 \mathrm{~min}$. The mixture was then extracted with $\mathrm{Et}_{2} \mathrm{O}$ (x 3), washed with brine and the combined organic layers were dried over $\mathrm{MgSO}_{4}$. After filtration and concentration under reduced pressure, the residue was purified by chromatography on silica gel (eluent: cyclohexane/AcOEt), affording fluorenones $\mathbf{4 a}(Z)$ and unreacted bromofluoroolefin 2(Z). 


\section{Compound $\mathbf{2}_{\mathbf{1}}(\mathbf{Z})^{7 \mathrm{a}}$}


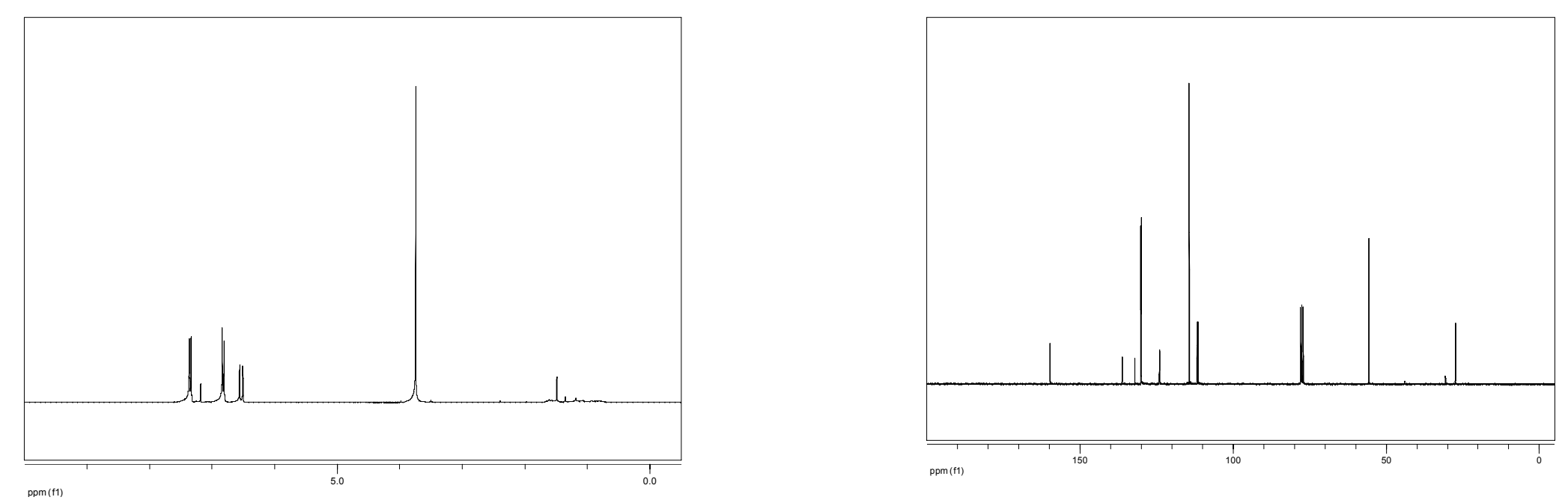


\section{Compound $\mathbf{4} \mathbf{a}_{1}(Z)$}

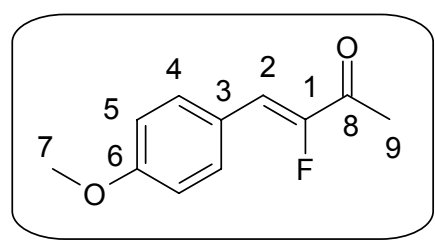

(Z) 3-Fluoro-4-(4-methoxyphenyl)but-3-en-2-one. Yellow powder (m. p. = 72$\left.74^{\circ} \mathrm{C}\right), R_{f} 0.2\left(5 \% \mathrm{AcOEt}\right.$ in cyclohexane). ${ }^{1} \mathrm{H}-\mathrm{NMR}\left(300 \mathrm{MHz}, \mathrm{CDCl}_{3}\right) \delta 7.6(\mathrm{~d}, \mathrm{~J}=$ $\left.8.9 \mathrm{~Hz}, 2 \mathrm{H}, \mathrm{H}_{4}\right), 6.9\left(\mathrm{~d}, \mathrm{~J}=8.9 \mathrm{~Hz}, 2 \mathrm{H}, \mathrm{H}_{5}\right), 6.7\left(\mathrm{~d},{ }^{3} \mathrm{~J}_{\mathrm{H}-\mathrm{F}}=36.9 \mathrm{~Hz}, 1 \mathrm{H}, \mathrm{H}_{2}\right), 3.7(\mathrm{~s}$, $\left.3 \mathrm{H}, \mathrm{H}_{7}\right), 2.3\left(\mathrm{~d},{ }^{4} \mathrm{~J}_{\mathrm{H}-\mathrm{F}}=3.3 \mathrm{~Hz}, 3 \mathrm{H}, \mathrm{H}_{9}\right) .{ }^{19} \mathrm{~F}-\mathrm{NMR}\left(282.5 \mathrm{MHz}, \mathrm{CDCl}_{3}\right) \delta-127.1$ (dq, $\left.{ }^{3} \mathrm{~J}_{\mathrm{H}-\mathrm{F}}=36.9 \mathrm{~Hz},{ }^{4} \mathrm{~J}_{\mathrm{H}-\mathrm{F}}=3.3 \mathrm{~Hz}\right) \cdot{ }^{13} \mathrm{C}-\mathrm{NMR}\left(75.5 \mathrm{MHz}, \mathrm{CDCl}_{3}\right) \delta 192.2\left(\mathrm{~d},{ }^{2} \mathrm{~J}_{\mathrm{C}-\mathrm{F}}=\right.$ $\left.32 \mathrm{~Hz}, \mathrm{C}_{8}\right), 161.2\left(\mathrm{~d}, \mathrm{~J}=4 \mathrm{~Hz}, \mathrm{C}_{6}\right), 153.5\left(\mathrm{~d},{ }^{1} \mathrm{~J}_{\mathrm{CF}}=267 \mathrm{~Hz}, \mathrm{C}_{1}\right), 135.5(\mathrm{~d}, \mathrm{~J}=6 \mathrm{~Hz}$ $\left.\mathrm{C}_{4}\right), 124.0\left(\mathrm{~d},{ }^{3} \mathrm{~J}_{\mathrm{C}-\mathrm{F}}=4 \mathrm{~Hz}, \mathrm{C}_{3}\right), 116.4\left(\mathrm{~d},{ }^{2} \mathrm{~J}_{\mathrm{C}-\mathrm{F}}=6 \mathrm{~Hz}, \mathrm{C}_{2}\right), 114.5\left(\mathrm{~d}, \mathrm{~J}=18 \mathrm{~Hz}, \mathrm{C}_{5}\right)$, $55.7\left(\mathrm{C}_{7}\right), 25.9\left(\mathrm{C}_{9}\right) . \mathrm{MS}(\mathrm{EI}) 194\left(\mathrm{M}^{+}\right), 179\left(\mathrm{M}^{+}-\mathrm{CH}_{3}\right), 159,43\left(\mathrm{CH}_{3} \mathrm{CO}^{+}\right)$. IR $(\mathrm{KBr})$ 3037, 2970, 2937, 2898, 2837, 1672, 1643, 1605, 1570, 1513, 1424, 1343, 1264, $1185,1177,1069,1022,869,808,539 \mathrm{~cm}^{-1}$. Anal. Calcd for $\mathrm{C}_{11} \mathrm{H}_{11} \mathrm{FO}_{2}: \mathrm{C}, 68.03$; $H, 5.71$. Found: C, 68.06; $H, 5.63$.
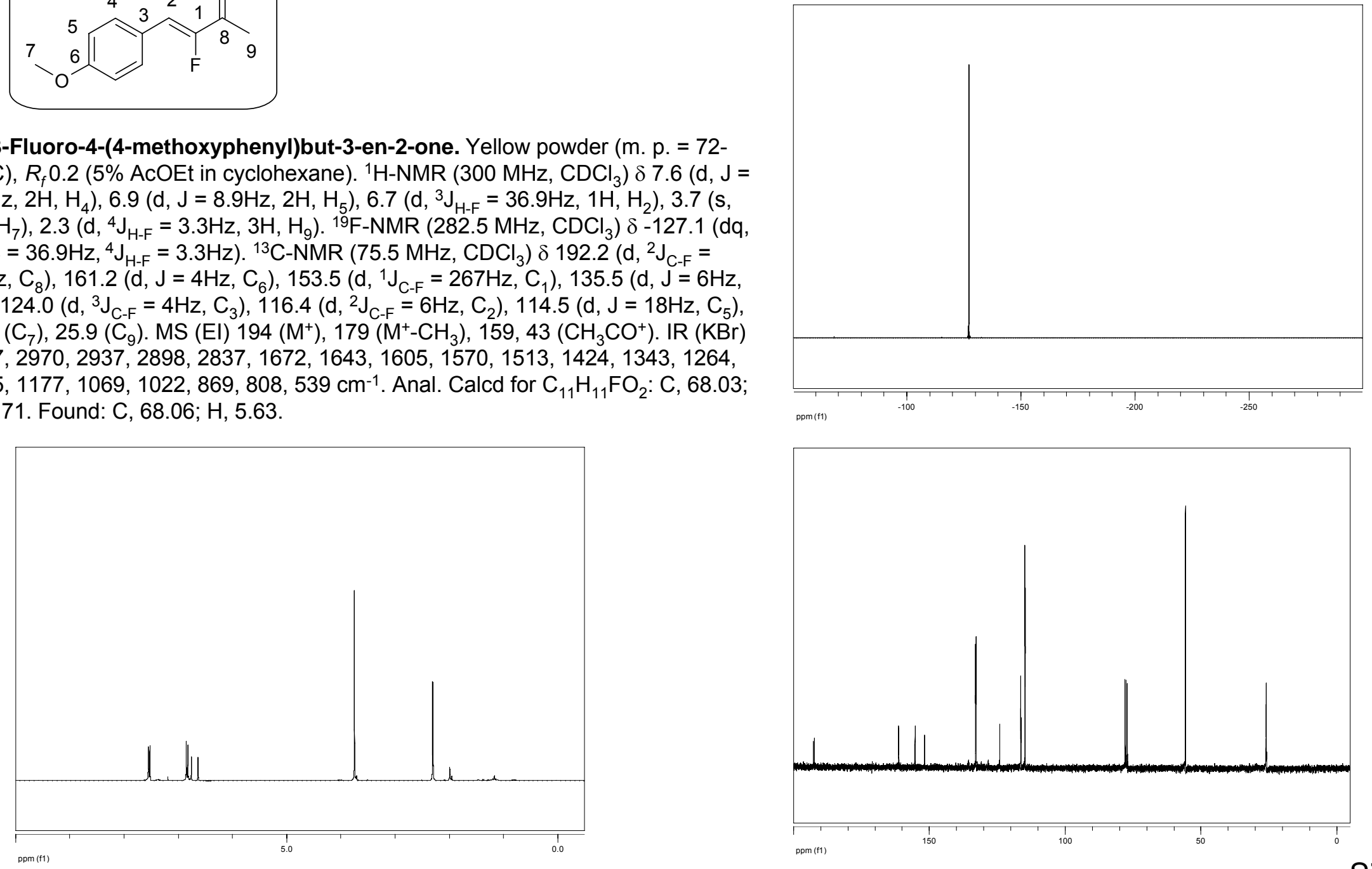


\section{Compound $\mathbf{2}_{\mathbf{2}}(Z)^{7 a}$}
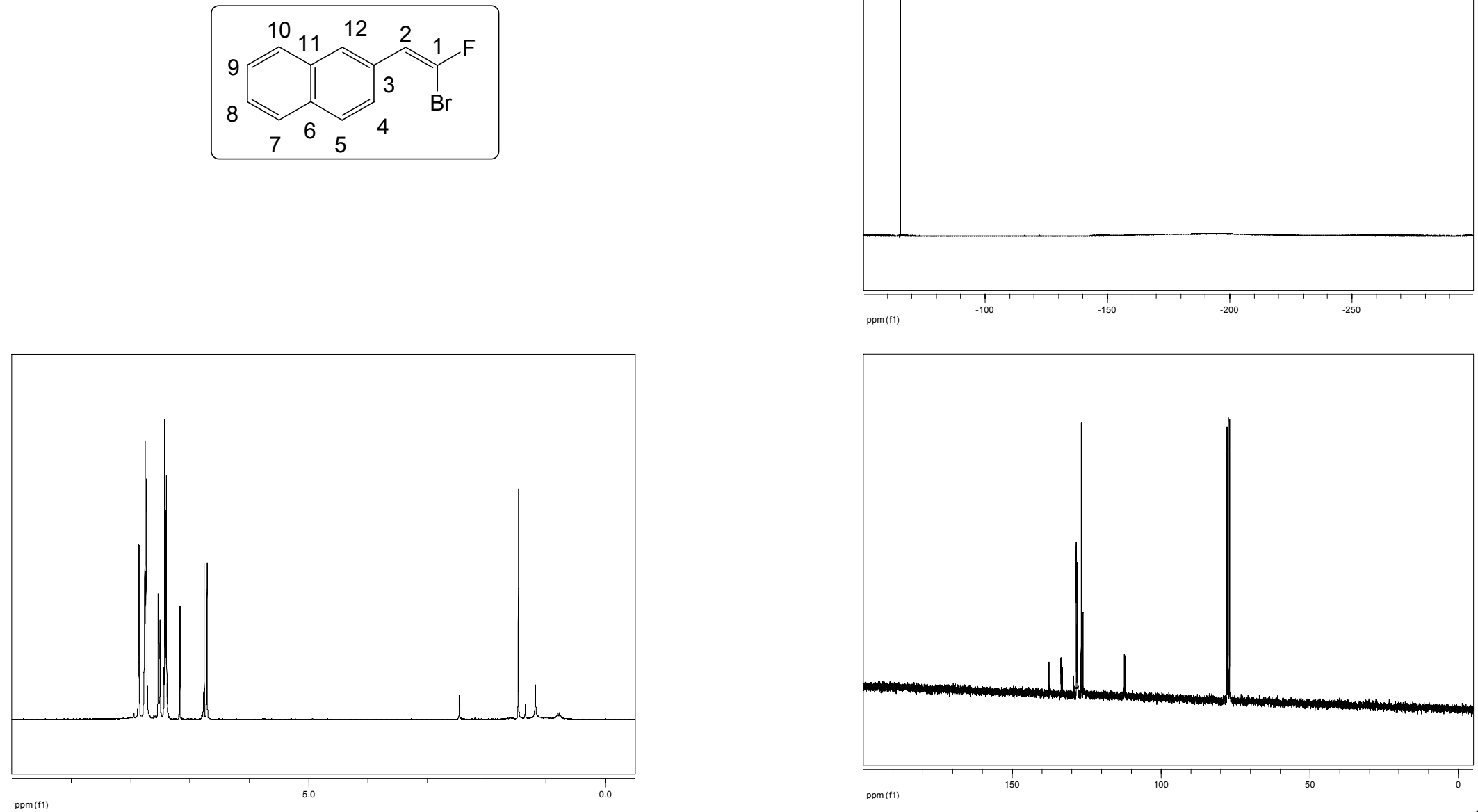


\section{Compound $\mathbf{4} \mathbf{a}_{2}(Z)$}

(Z) 3-Fluoro-4-(naphthalen-7-yl)but-3-en-2-one. Yellow powder (m. p. $=85-87^{\circ} \mathrm{C}$ ), $R_{f} 0.3$ (5\% AcOEt in cyclohexane). ${ }^{1} \mathrm{H}-\mathrm{NMR}\left(300 \mathrm{MHz}, \mathrm{CDCl}_{3}\right) \delta 8.0\left(\mathrm{~s}, 1 \mathrm{H}, \mathrm{H}_{12}\right)$, 7.8-7.6 (m, 4H, $\mathrm{H}_{7,8.9}$ 10), 7.5-7.3 (m, 2H, $\left.\mathrm{H}_{4,5}\right), 6.9\left(\mathrm{~d},{ }^{3} \mathrm{~J}_{\mathrm{H}-\mathrm{F}}=36.6 \mathrm{~Hz}, 1 \mathrm{H}, \mathrm{H}_{2}\right), 2.3$ $\left(\mathrm{d},{ }^{4} \mathrm{~J}_{\mathrm{H}-\mathrm{F}}=3.3 \mathrm{~Hz}, 3 \mathrm{H}, \mathrm{H}_{14}\right) .{ }^{19} \mathrm{~F}-\mathrm{NMR}\left(282.5 \mathrm{MHz}, \mathrm{CDCl}_{3}\right) \delta-123.8\left(\mathrm{dq},{ }^{3} \mathrm{~J}_{\mathrm{H}-\mathrm{F}}=\right.$ $\left.36.6 \mathrm{~Hz},{ }^{4} \mathrm{~J}_{\mathrm{H}-\mathrm{F}}=3.3 \mathrm{~Hz}\right) .{ }^{13} \mathrm{C}-\mathrm{NMR}\left(75.5 \mathrm{MHz}, \mathrm{CDCl}_{3}\right) \delta 192.5\left(\mathrm{~d},{ }^{2} \mathrm{~J}_{\mathrm{C}-\mathrm{F}}=33 \mathrm{~Hz}, \mathrm{C}_{13}\right)$, $154.5\left(\mathrm{~d},{ }^{1} \mathrm{~J}_{\mathrm{C}-\mathrm{F}}=272 \mathrm{~Hz}, \mathrm{C}_{1}\right), 134.1\left(\mathrm{~d}, \mathrm{~J}=2 \mathrm{~Hz}, \mathrm{C}^{9}{ }^{9}\right), 133.6\left(\mathrm{C}_{\mathrm{a}}{ }^{\mathrm{q}}\right), 131.8(\mathrm{~d}, \mathrm{~J}=8 \mathrm{~Hz}$, $\left.\mathrm{C}_{\mathrm{ar}}{ }^{\mathrm{H}}\right), 129.03\left(\mathrm{C}_{\mathrm{ar}}{ }^{\mathrm{H}}\right), 129.0\left(\mathrm{C}_{\mathrm{ar}}{ }^{\mathrm{q}}\right), 128.9\left(\mathrm{C}_{\mathrm{ar}}{ }^{\mathrm{H}}\right), 128.1\left(\mathrm{C}_{\mathrm{ar}}{ }^{\mathrm{H}}\right), 127.8\left(\mathrm{C}_{\mathrm{ar}}{ }^{\mathrm{H}}\right), 127.4(\mathrm{~d}, \mathrm{~J}$ $\left.=9 \mathrm{~Hz}, \mathrm{C}_{\mathrm{ar}}{ }^{\mathrm{H}}\right), 127.0\left(\mathrm{C}_{\mathrm{ar}}^{\mathrm{H}}\right), 116.2\left(\mathrm{~d},{ }^{2} \mathrm{~J}_{\mathrm{C}-\mathrm{F}}=6 \mathrm{~Hz}, \mathrm{C}_{2}\right), 26.1\left(\mathrm{C}_{14}\right) . \mathrm{MS}(\mathrm{EI}) 214\left(\mathrm{M}^{+}\right)$, $199\left(\mathrm{M}^{+}-\mathrm{CH}_{3}\right), 179,171\left(\mathrm{M}^{+}-\mathrm{CH}_{3} \mathrm{CO}\right), 43\left(\mathrm{CH}_{3} \mathrm{CO}^{+}\right) . \mathrm{IR}(\mathrm{KBr}) \delta 3050,1702,1683$, 1626, 1365, 1335, 1320, 1243, 1069, 914, 829, 751, 625, 483. Anal. Calcd for $\mathrm{C}_{14} \mathrm{H}_{11} \mathrm{FO}: \mathrm{C}, 78.49 ; \mathrm{H}, 5.18$. Found: C, 78.57; H, 5.15.
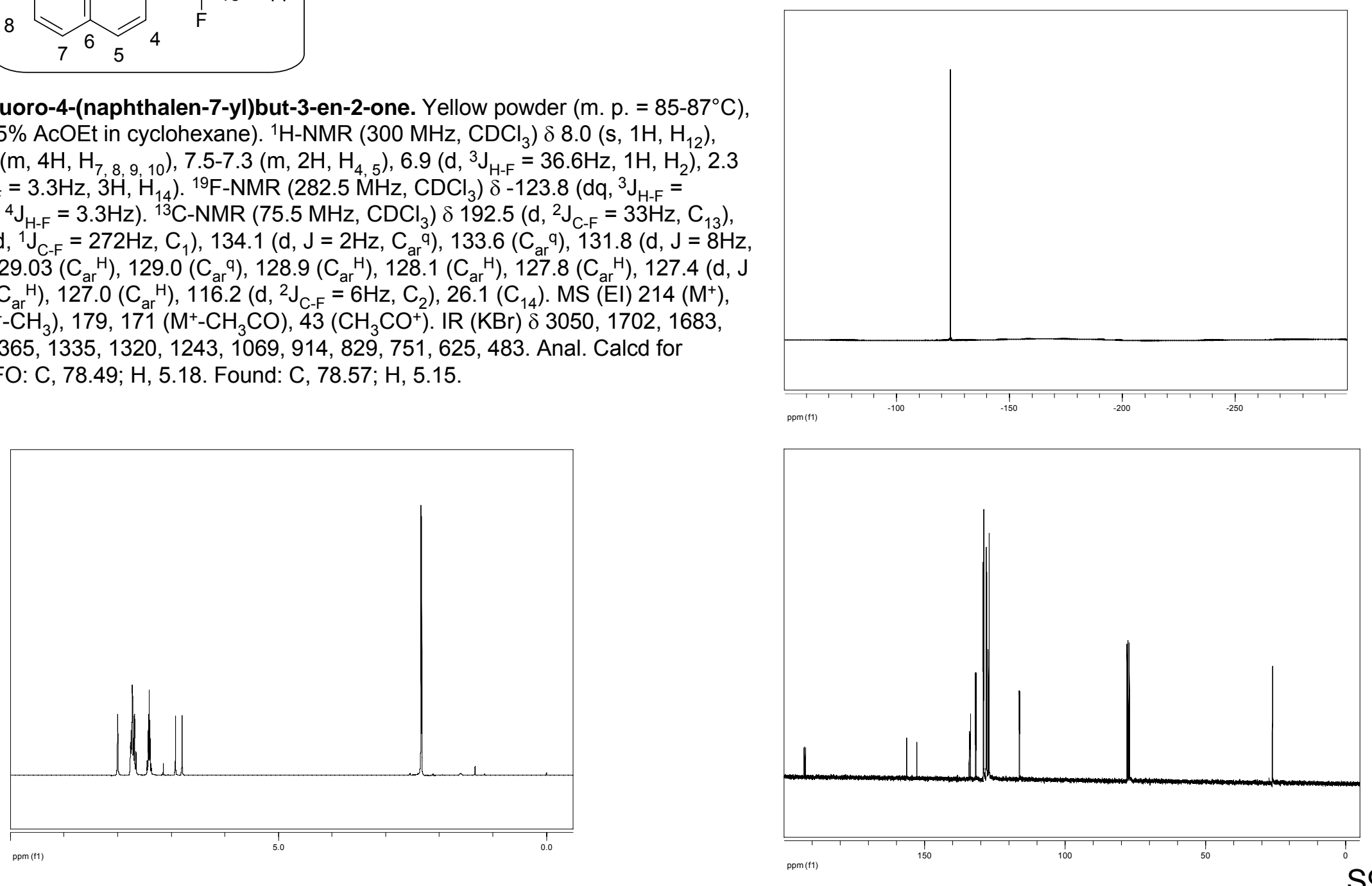


\section{Compound $\mathbf{2}_{3}(Z)^{7 a}$}
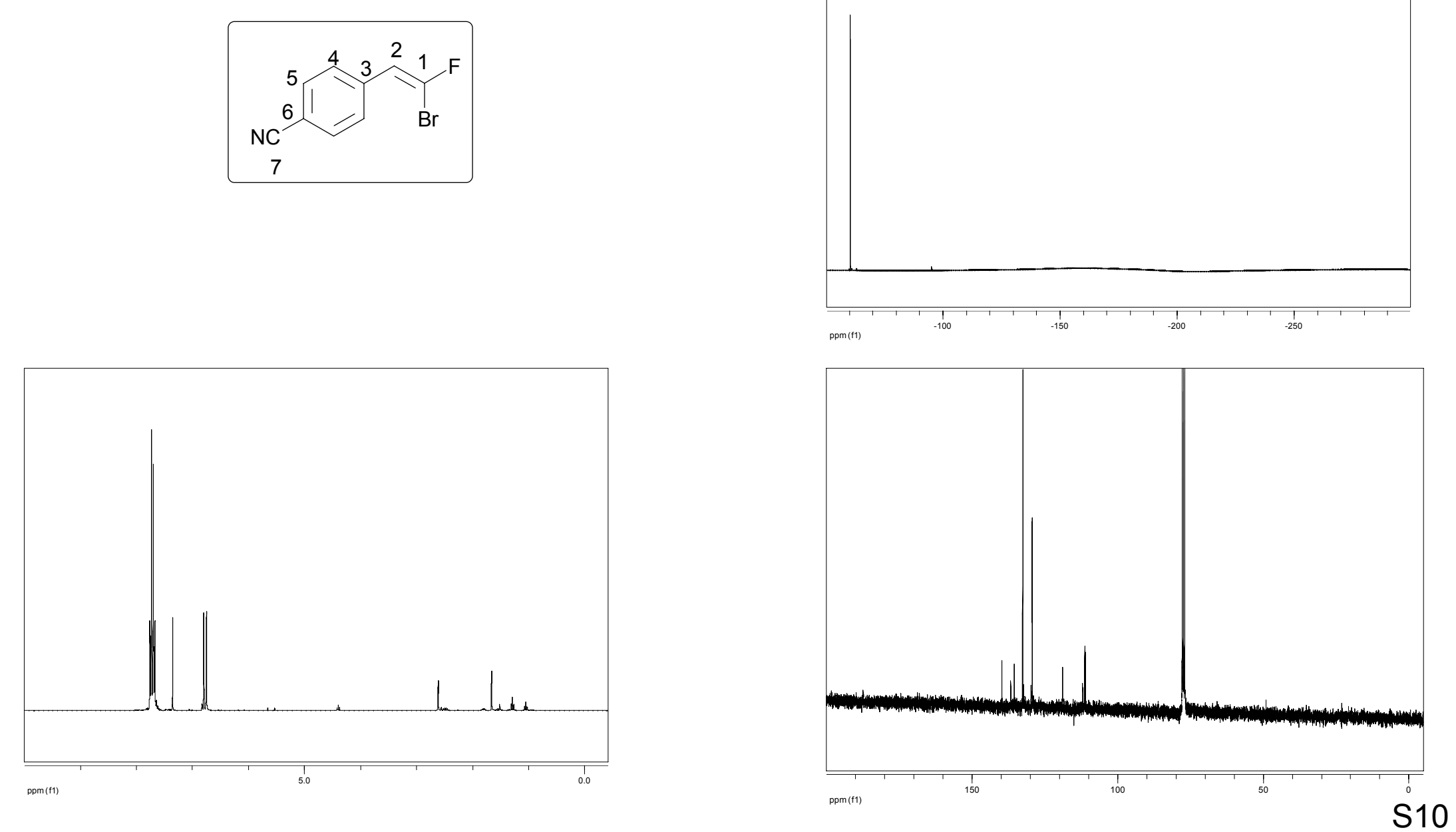


\section{Compound 4a3(Z)}

(Z) 3-Fluoro-4-(4-cyanophenyl)but-3-en-2-one. Yellow powder (m. p. $=119$ -

$\left.121^{\circ} \mathrm{C}\right), R_{f} 0.3\left(5 \%\right.$ AcOEt in cyclohexane). ${ }^{1} \mathrm{H}-\mathrm{NMR}\left(300 \mathrm{MHz}, \mathrm{CDCl}_{3}\right) \delta 7.6(\mathrm{~d}, \mathrm{~J}=$ $\left.8.5 \mathrm{~Hz}, 2 \mathrm{H}, \mathrm{H}_{5}\right), 7.1\left(\mathrm{~d}, \mathrm{~J}=8.5 \mathrm{~Hz}, 2 \mathrm{H}, \mathrm{H}_{4}\right), 6.8\left(\mathrm{~d},{ }^{3} \mathrm{~J}_{\mathrm{H}-\mathrm{F}}=35.3 \mathrm{~Hz}, 1 \mathrm{H}, \mathrm{H}_{2}\right), 2.4\left(\mathrm{~d},{ }^{4} \mathrm{~J}_{\mathrm{H}-}\right.$ $\left.\mathrm{F}=3.3 \mathrm{~Hz}, 3 \mathrm{H}, \mathrm{H}_{9}\right) \cdot{ }^{19} \mathrm{~F}-\mathrm{NMR}\left(282.5 \mathrm{MHz}, \mathrm{CDCl}_{3}\right) \delta-119.6\left(\mathrm{dq},{ }^{3} \mathrm{~J}_{\mathrm{H}-\mathrm{F}}=35.3 \mathrm{~Hz},{ }^{4} \mathrm{~J}_{\mathrm{H}-\mathrm{F}}\right.$ $=3.3 \mathrm{~Hz}) .{ }^{13} \mathrm{C}-\mathrm{NMR}\left(75.5 \mathrm{MHz}, \mathrm{CDCl}_{3}\right) \delta 192.4\left(\mathrm{~d},{ }^{2} \mathrm{~J}_{\mathrm{C}-\mathrm{F}}=35 \mathrm{~Hz}, \mathrm{C}_{8}\right), 155.5\left(\mathrm{~d},{ }^{1} \mathrm{~J}_{\mathrm{C}-\mathrm{F}}\right.$ $\left.=278 \mathrm{~Hz}, \mathrm{C}_{1}\right), 135.8\left(\mathrm{~d},{ }^{3} \mathrm{~J}_{\mathrm{C}-\mathrm{F}}=4 \mathrm{~Hz}, \mathrm{C}_{3}\right), 132.9\left(\mathrm{C}_{5}\right), 131.2\left(\mathrm{~d}, \mathrm{~J}=9 \mathrm{~Hz}, \mathrm{C}_{4}\right), 118.7$ $\left(\mathrm{C}_{7}\right), 113.4\left(\mathrm{~d},{ }^{2} \mathrm{~J}_{\mathrm{C}-\mathrm{F}}=5 \mathrm{~Hz}, \mathrm{C}_{2}\right), 113.3\left(\mathrm{~d}, \mathrm{~J}=4 \mathrm{~Hz}, \mathrm{C}_{6}\right), 26.1\left(\mathrm{C}_{9}\right) . \mathrm{MS}(\mathrm{EI}) 189\left(\mathrm{M}^{+}\right)$, $174\left(\mathrm{M}^{+}-\mathrm{CH}_{3}\right), 146\left(\mathrm{M}^{+}-\mathrm{CH}_{3} \mathrm{CO}\right), 43\left(\mathrm{CH}_{3} \mathrm{CO}^{+}\right)$. IR $(\mathrm{KBr}) 3075,2987,2227,1681$, $1648,1505,1418,1382,1351,1328,1293,1255,1212,1081,968,834,616$ 561.Anal. Calcd for $\mathrm{C}_{11} \mathrm{H}_{8} \mathrm{FNO}: \mathrm{C}, 69.84 ; \mathrm{H}, 4.26 ; \mathrm{N}, 7.40$. Found : C, 69.67; $\mathrm{H}$, 4.06; N, 7.24 .
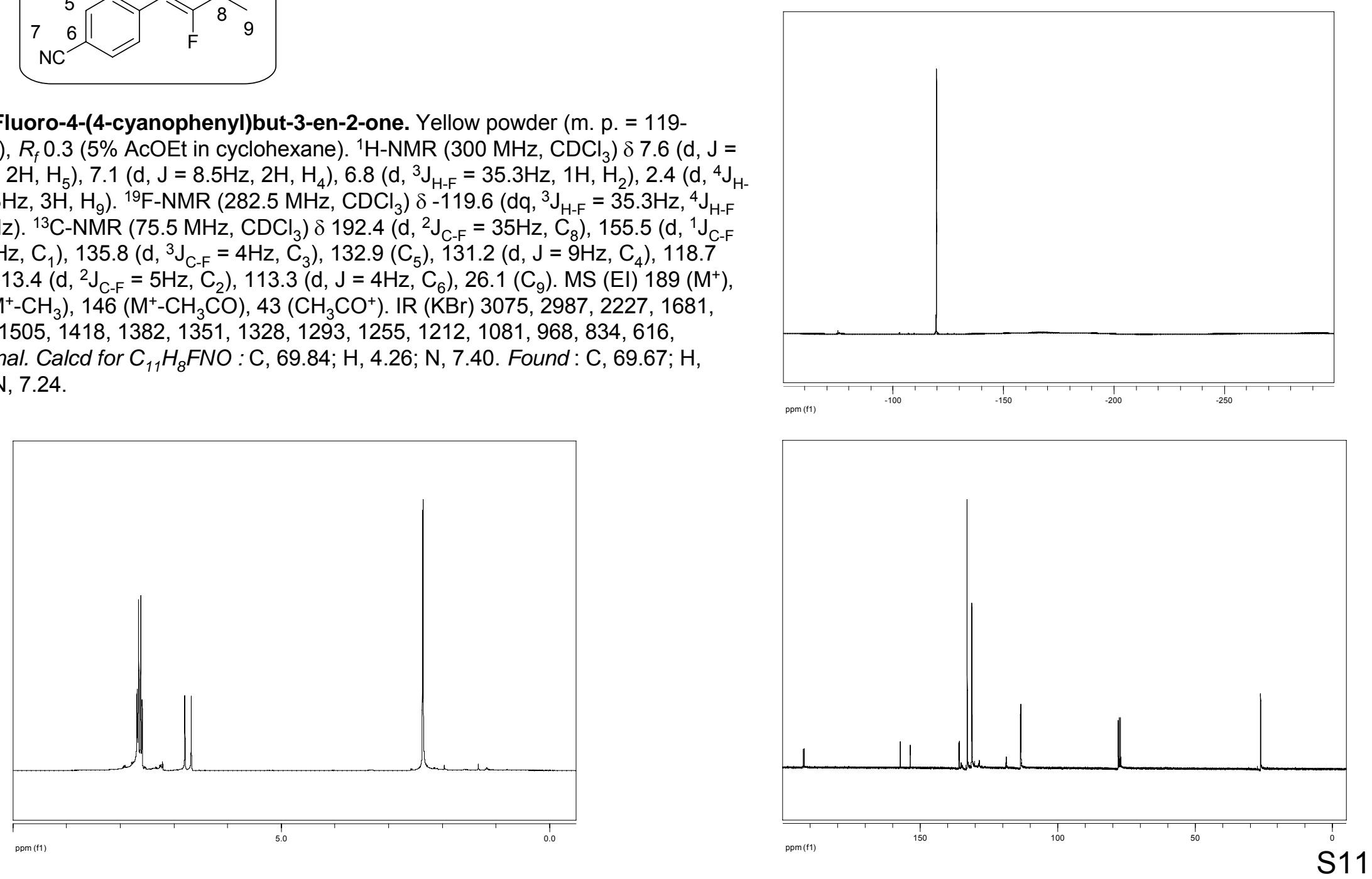


\section{Compound $\mathbf{2}_{\mathbf{4}}(\mathbf{Z})^{7 \mathrm{a}}$}
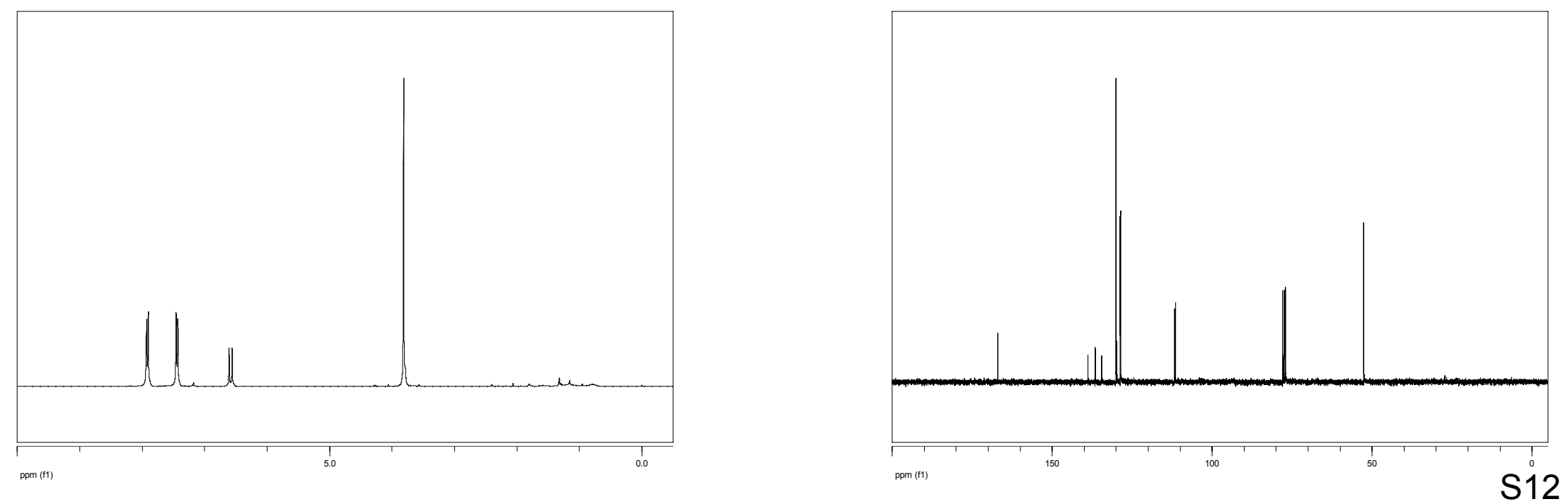


\section{Compound $\mathbf{4} \mathbf{a}_{4}(\mathbf{Z})$}

(Z) 3-Fluoro-4-(4-methylbenzoate)but-3-en-2-one. Yellow solid (m. p. = 103$\left.105^{\circ} \mathrm{C}\right), R_{f} 0.2\left(5 \%\right.$ AcOEt in cyclohexane). ${ }^{1} \mathrm{H}-\mathrm{NMR}\left(300 \mathrm{MHz}^{\mathrm{C}} \mathrm{CDCl}_{3}\right) \delta 8.0(\mathrm{~d}, \mathrm{~J}=$ $\left.8.5 \mathrm{~Hz}, 2 \mathrm{H}, \mathrm{H}_{5}\right), 7.6\left(\mathrm{~d}, \mathrm{~J}=8.5 \mathrm{~Hz}, 2 \mathrm{H}, \mathrm{H}_{4}\right), 6.8\left(\mathrm{~d},{ }^{3} \mathrm{~J}_{\mathrm{H}-\mathrm{F}}=35.8 \mathrm{~Hz}, 1 \mathrm{H}, \mathrm{H}_{2}\right), 3.9(\mathrm{~s}$, $\left.3 \mathrm{H}, \mathrm{H}_{8}\right), 2.4\left(\mathrm{~d},{ }^{4} \mathrm{~J}_{\mathrm{H}-\mathrm{F}}=3.6 \mathrm{~Hz}, 3 \mathrm{H}, \mathrm{H}_{10}\right)$. ${ }^{19} \mathrm{~F}-\mathrm{NMR}\left(282.5 \mathrm{MHz}, \mathrm{CDCl}_{3}\right) \delta-121.1$ (dq, $\left.{ }^{3} \mathrm{~J}_{\mathrm{H}-\mathrm{F}}=35.8 \mathrm{~Hz},{ }^{4} \mathrm{~J}_{\mathrm{H}-\mathrm{F}}=3.6 \mathrm{~Hz}\right) \cdot{ }^{13} \mathrm{C}-\mathrm{NMR}\left(75.5 \mathrm{MHz}, \mathrm{CDCl}_{3}\right) \delta 192.7\left(\mathrm{~d},{ }^{2} \mathrm{~J}_{\mathrm{C}-\mathrm{F}}=\right.$ $\left.35 \mathrm{~Hz}, \mathrm{C}_{9}\right), 166.8\left(\mathrm{C}_{7}\right), 155.1\left(\mathrm{~d},{ }^{1} \mathrm{~J}_{\mathrm{C}-\mathrm{F}}=277 \mathrm{~Hz}, \mathrm{C}_{1}\right), 135.7\left(\mathrm{~d}, \mathrm{~J}=4 \mathrm{~Hz}, \mathrm{C}_{6}\right), 131.2$ $\left(\mathrm{d},{ }^{3} \mathrm{~J}_{\mathrm{C}-\mathrm{F}}=3 \mathrm{~Hz}, \mathrm{C}_{3}\right), 130.8\left(\mathrm{~d}, \mathrm{~J}=9 \mathrm{~Hz}, \mathrm{C}_{4}\right), 130.3\left(\mathrm{C}_{5}\right), 114.4\left(\mathrm{~d},{ }^{2} \mathrm{~J}_{\mathrm{C}-\mathrm{F}}=6 \mathrm{~Hz}, \mathrm{C}_{2}\right)$, $52.7\left(\mathrm{C}_{8}\right), 26.2\left(\mathrm{C}_{10}\right)$. MS (El) $222\left(\mathrm{M}^{+}\right), 207\left(\mathrm{M}^{+}-\mathrm{CH}_{3}\right), 191\left(\mathrm{M}^{+}-\mathrm{CH}_{3} \mathrm{O}\right), 163\left(\mathrm{M}^{+}-\right.$ $\left.\mathrm{CO}_{2} \mathrm{CH}_{3}\right), 43\left(\mathrm{CH}_{3} \mathrm{CO}^{+}\right)$. IR (KBr) 3056, 2959, 1721, 1636, 1433, 1364, 1320, 1280 , 1192, 1109, 1057, 1019, 968, 904, 832, 769, 697, 571.Anal. Calcd for $\mathrm{C}_{12} \mathrm{H}_{11} \mathrm{FO}_{3}$ : C, 64.86; H, 4.99. Found : C, 64.98; H, 4.90.
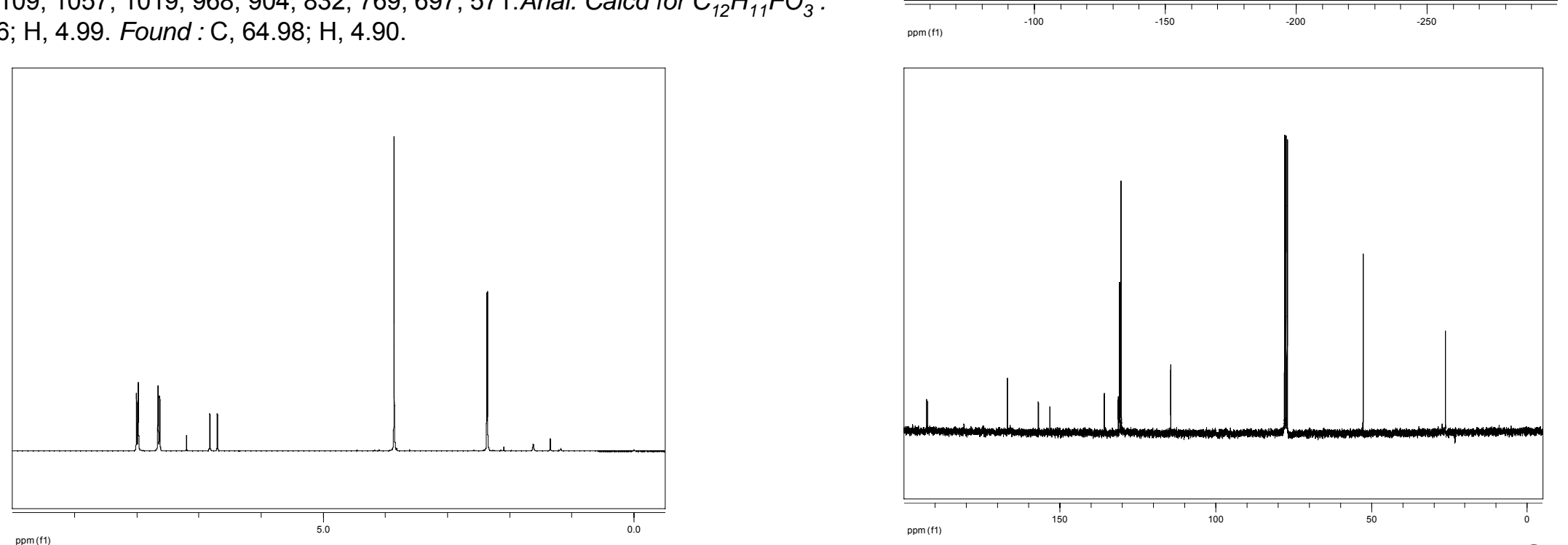


\section{Compound $\mathbf{2}_{5}(\mathbf{Z})^{7 \mathrm{a}}$}
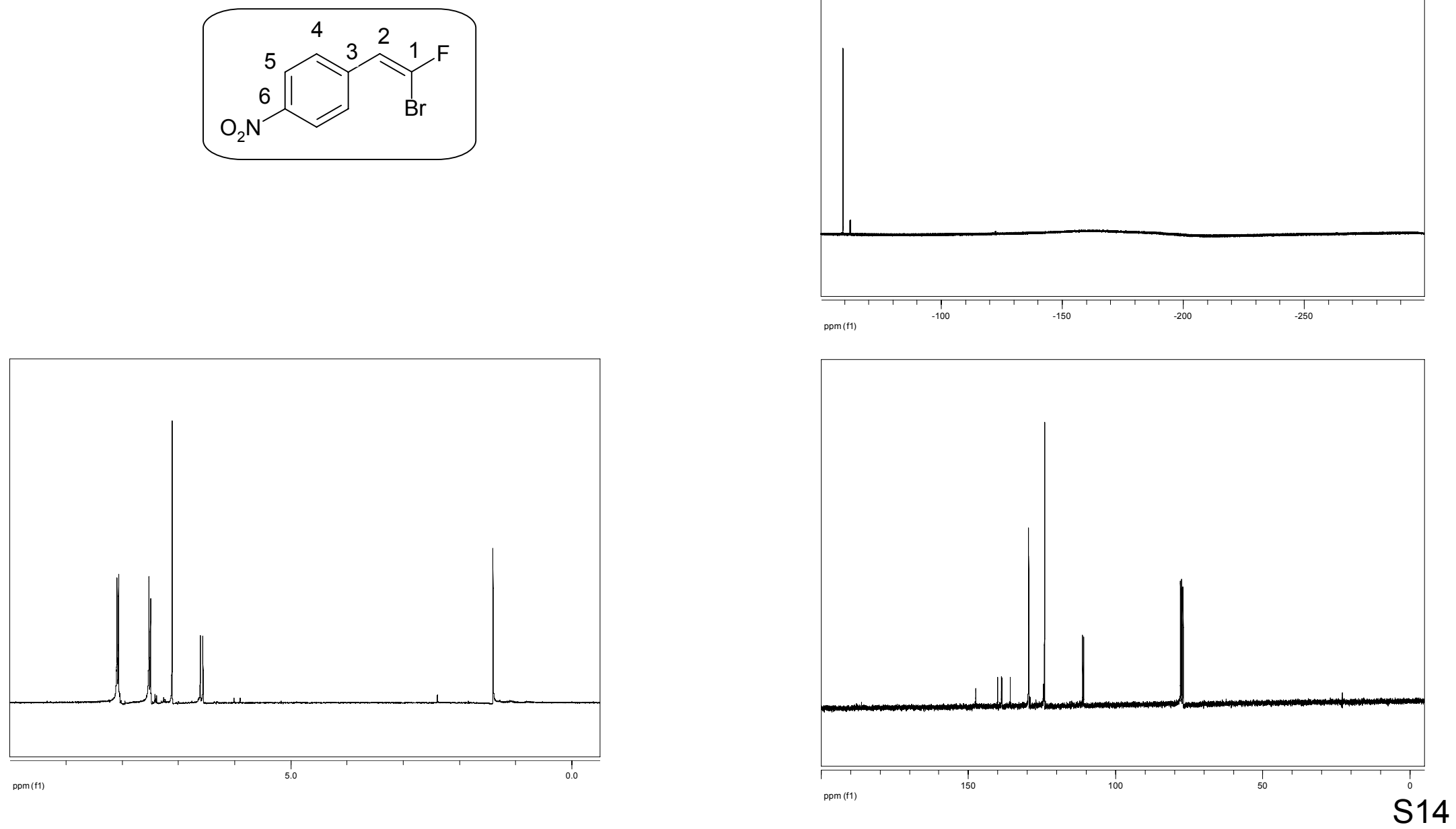


\section{Compound $\mathbf{4} \mathbf{a}_{5}(\mathbf{Z})$}

(Z) 3-Fluoro-4-(4-nitrophenyl)but-3-en-2-one. Yellow solid (m. p. $\left.=139-141^{\circ} \mathrm{C}\right), R_{f}$ 0.1 (5\% AcOEt in cyclohexane). ${ }^{1} \mathrm{H}-\mathrm{NMR}\left(300 \mathrm{MHz}^{\mathrm{C}} \mathrm{CDCl}_{3}\right) \delta 8.2(\mathrm{~d}, \mathrm{~J}=8.8 \mathrm{~Hz}, 2 \mathrm{H}$, $\left.\mathrm{H}_{5}\right), 7.8\left(\mathrm{~d}, \mathrm{~J}=8.8 \mathrm{~Hz}, 2 \mathrm{H}, \mathrm{H}_{4}\right), 6.8\left(\mathrm{~d},{ }^{3} \mathrm{~J}_{\mathrm{H}-\mathrm{F}}=35.0 \mathrm{~Hz}, 1 \mathrm{H}, \mathrm{H}_{2}\right), 2.5\left(\mathrm{~d},{ }^{4} \mathrm{~J}_{\mathrm{H}-\mathrm{F}}=3.8 \mathrm{~Hz}\right.$, $\left.3 \mathrm{H}, \mathrm{H}_{8}\right) .{ }^{19} \mathrm{~F}-\mathrm{NMR}\left(282.5 \mathrm{MHz}^{\mathrm{C} C \mathrm{CDI}_{3}}\right) \delta-119.2\left(\mathrm{dq},{ }^{3} \mathrm{~J}_{\mathrm{H}-\mathrm{F}}=35.5 \mathrm{~Hz},{ }^{4} \mathrm{~J}_{\mathrm{H}-\mathrm{F}}=3.3 \mathrm{~Hz}\right)$. ${ }^{13} \mathrm{C}-N M R\left(75.5 \mathrm{MHz}, \mathrm{CDCl}_{3}\right) \delta 192.2\left(\mathrm{~d},{ }^{2} \mathrm{~J}_{\mathrm{C}-\mathrm{F}}=34 \mathrm{~Hz}, \mathrm{C}_{7}\right), 155.3\left(\mathrm{~d},{ }^{1} \mathrm{~J}_{\mathrm{C}-\mathrm{F}}=280 \mathrm{~Hz}\right.$, $\left.\mathrm{C}_{1}\right), 147.9\left(\mathrm{~d}, \mathrm{~J}=3 \mathrm{~Hz}, \mathrm{C}_{6}\right), 137.3\left(\mathrm{~d}, \mathrm{~J}=4 \mathrm{~Hz}, \mathrm{C}_{3}\right), 131.3\left(\mathrm{~d}, \mathrm{~J}=13 \mathrm{~Hz}, \mathrm{C}_{5}\right), 123.3$ $\left(\mathrm{C}_{4}\right), 117.5\left(\mathrm{~d},{ }^{2} \mathrm{~J}_{\mathrm{C}-\mathrm{F}}=5 \mathrm{~Hz}, \mathrm{C}_{2}\right), 25.9\left(\mathrm{C}_{8}\right) . \mathrm{MS}(\mathrm{El}) 209\left(\mathrm{M}^{+}\right), 192,162\left(\mathrm{M}^{+}-\mathrm{HNO}_{2}\right)$, 148. IR (KBr) 3306, 3007, 1707, 1639, 1595, 1511, 1340, 1304, 1268, 1200, 690. Anal. Calcd for $\mathrm{C}_{10} \mathrm{H}_{8} \mathrm{FNO}_{3}$ : C, 57.42; $\mathrm{H}, 3.85 ; \mathrm{N}, 6.70$. Found: $\mathrm{C}, 57.78 ; \mathrm{H}, 3.79 ; \mathrm{N}$, 6.64 .



\section{Compound $\mathbf{2}_{\mathbf{6}}(\mathbf{Z})^{7 \mathrm{a}}$}
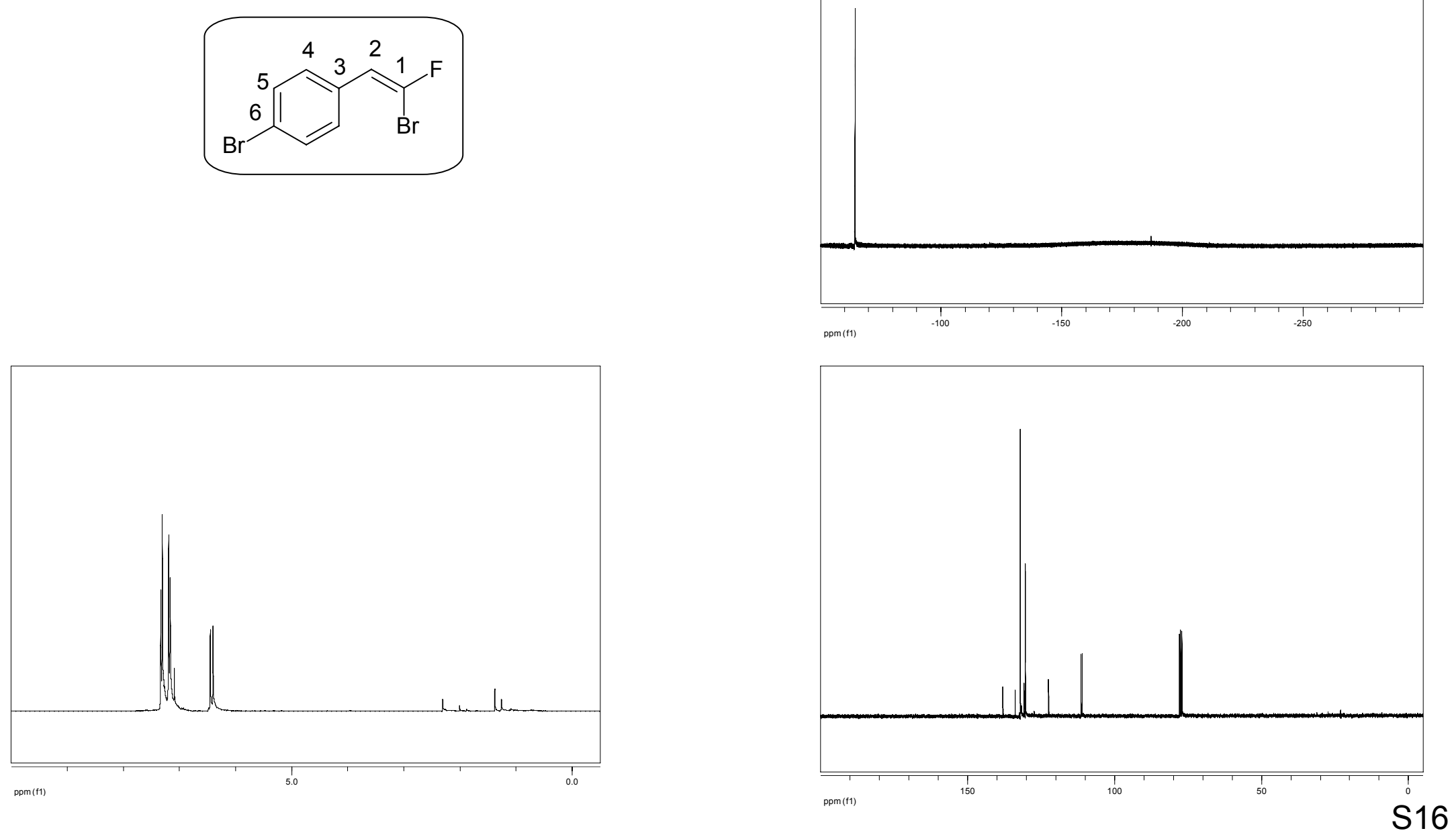


\section{Compound $\mathbf{4} \mathbf{a}_{6}(Z)$}

(Z) 3-Fluoro-4-(4-bromophenyl)but-3-en-2-one. Yellow solid (m. p. $=88-90^{\circ} \mathrm{C}$ ), $R_{f}$

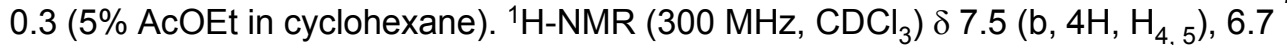
$\left(\mathrm{d},{ }^{3} \mathrm{~J}_{\mathrm{H}-\mathrm{F}}=35.8 \mathrm{~Hz}, 1 \mathrm{H}, \mathrm{H}_{2}\right), 2.4\left(\mathrm{~d},{ }^{4} \mathrm{~J}_{\mathrm{H}-\mathrm{F}}=3.6 \mathrm{~Hz}, 3 \mathrm{H}, \mathrm{H}_{8}\right) .{ }^{19} \mathrm{~F}-\mathrm{NMR}(282.5 \mathrm{MHz}$, $\left.\mathrm{CDCl}_{3}\right) \delta-122.9\left(\mathrm{dq},{ }^{3} \mathrm{~J}_{\mathrm{H}-\mathrm{F}}=35.5 \mathrm{~Hz},{ }^{4} \mathrm{~J}_{\mathrm{H}-\mathrm{F}}=3.2 \mathrm{~Hz}\right) .{ }^{13} \mathrm{C}-\mathrm{NMR}\left(75.5 \mathrm{MHz}, \mathrm{CDCl}_{3}\right) \delta$ $192.3\left(\mathrm{~d},{ }^{2} \mathrm{~J}_{\mathrm{C}-\mathrm{F}}=34 \mathrm{~Hz}, \mathrm{C}_{7}\right), 154.2\left(\mathrm{~d}, 1 \mathrm{~J}_{\mathrm{C}-\mathrm{F}}=274 \mathrm{~Hz}, \mathrm{C}_{1}\right), 132.2\left(\mathrm{C}_{5}\right), 132.0(\mathrm{~d}, \mathrm{~J}=$ $\left.9 \mathrm{~Hz}, \mathrm{C}_{4}\right), 130.0\left(\mathrm{~d},{ }^{3} \mathrm{~J}_{\mathrm{C}-\mathrm{F}}=5 \mathrm{~Hz}, \mathrm{C}_{3}\right), 124.4\left(\mathrm{~d}, \mathrm{~J}=4 \mathrm{~Hz}, \mathrm{C}_{6}\right), 114.3\left(\mathrm{~d},{ }^{2} \mathrm{~J}_{\mathrm{C}-\mathrm{F}}=6 \mathrm{~Hz}\right.$, $\left.\mathrm{C}_{2}\right), 25.8\left(\mathrm{C}_{8}\right)$. MS (El) 244-242( $\left(\mathrm{M}^{+}\right), 163\left(\mathrm{M}^{+}-\mathrm{Br}\right), 148\left(\mathrm{M}^{+}-\mathrm{Br}_{2} \mathrm{CH}_{3}\right), 120\left(\mathrm{M}^{+}-\right.$ $\left.\mathrm{Br} \& \mathrm{COCH}_{3}\right)$. IR (KBr) 3021, 2959, 1700, 1634, 1360, 1310, 1267, 1197, 1056, 819. Anal. Calcd for $\mathrm{C}_{10} \mathrm{H}_{8}$ BrFO: C, 49.41; H, 3.32. Found: C, 49.52; $\mathrm{H}, 3.44$.



\section{Compound $\mathbf{2}_{7}(Z)^{7 a}$}

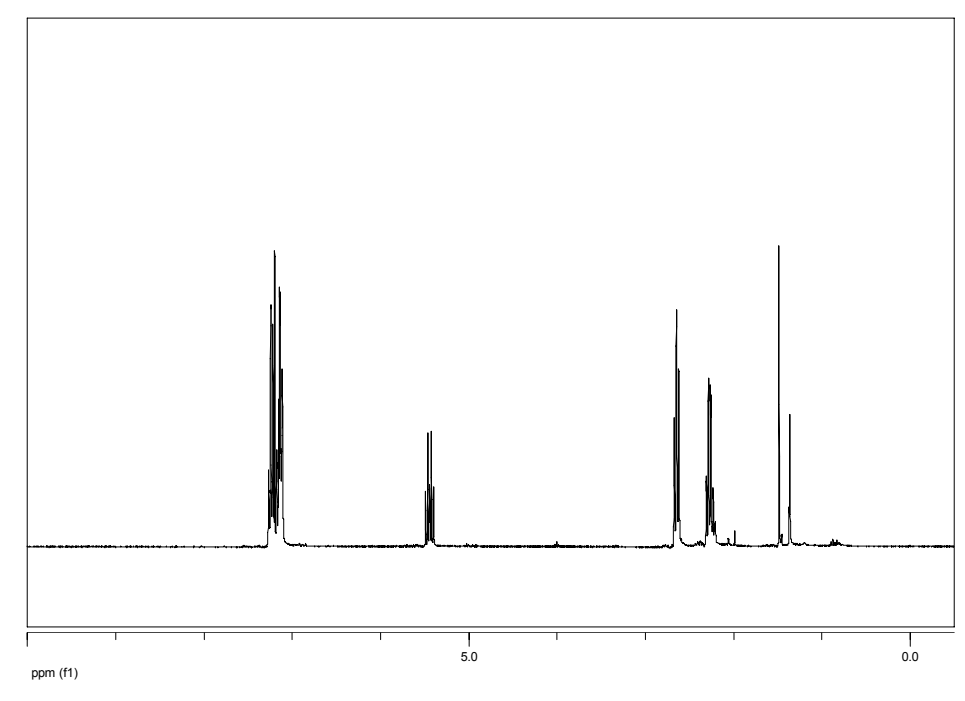

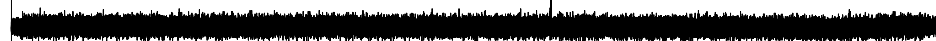
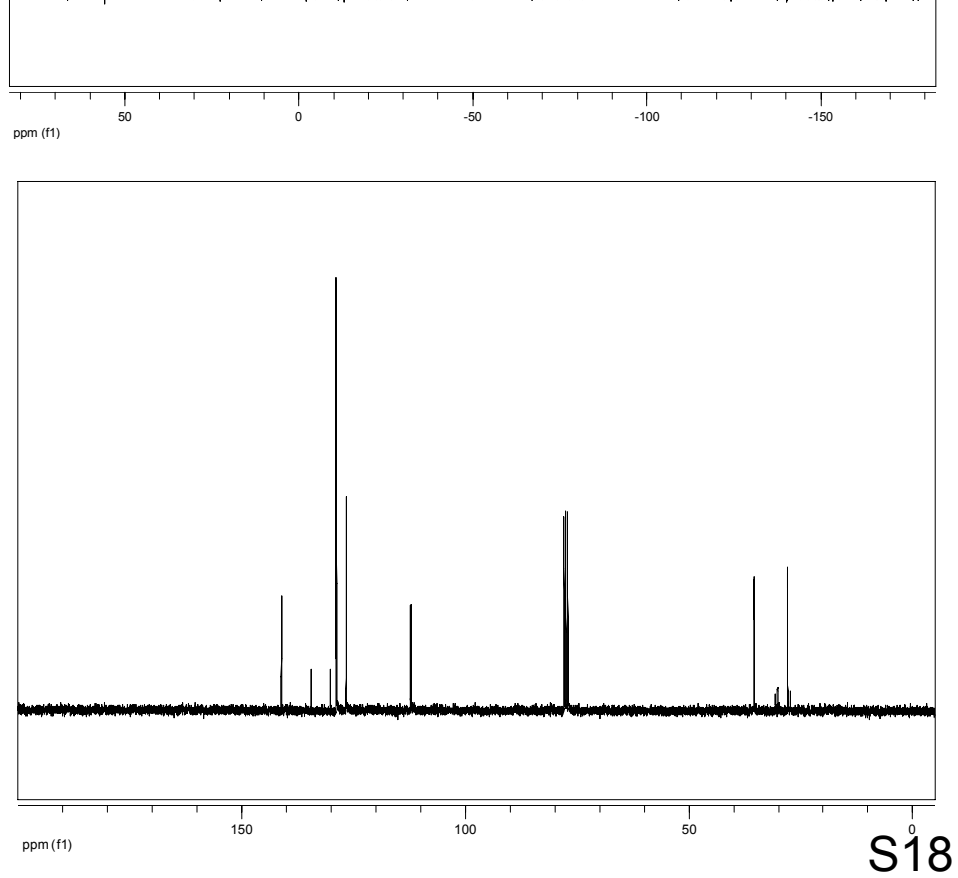


\section{Compound $\mathbf{4} \mathbf{a}_{7}(Z)$}

(Z) 3-Fluoro-6-phenylhex-3-en-2-one. Yellow oil, $R_{f} 0.3$ (5\% AcOEt in cyclohexane). ${ }^{1} \mathrm{H}-\mathrm{NMR}\left(300 \mathrm{MHz}, \mathrm{CDCl}_{3}\right) \delta 7.3-7.0\left(\mathrm{~m}, 5 \mathrm{H}, \mathrm{H}_{6,7,8}\right), 6.0\left(\mathrm{dt},{ }^{3} \mathrm{~J}_{\mathrm{H}-\mathrm{F}}\right.$ $\left.=33.8 \mathrm{~Hz},{ }^{3} \mathrm{~J}_{\mathrm{H}-\mathrm{H}}=7.4 \mathrm{~Hz}, 1 \mathrm{H}, \mathrm{H}_{2}\right), 2.7\left(\mathrm{t},{ }^{3} \mathrm{~J}_{\mathrm{H}-\mathrm{H}}=7.4 \mathrm{~Hz}, 2 \mathrm{H}, \mathrm{H}_{4}\right), 2.6-2.4(\mathrm{~m}, 2 \mathrm{H}$, $\left.\mathrm{H}_{3}\right), 2.3\left(\mathrm{dd},{ }^{4} \mathrm{~J}_{\mathrm{H}-\mathrm{F}}=2.8 \mathrm{~Hz}, \mathrm{~J}=0.8 \mathrm{~Hz}, 3 \mathrm{H}, \mathrm{H}_{10}\right) .{ }^{19} \mathrm{~F}-\mathrm{NMR}\left(282.5 \mathrm{MHz}, \mathrm{CDCl}_{3}\right) \delta-$ $127.8\left(\mathrm{dq},{ }^{3} \mathrm{~J}_{\mathrm{H}-\mathrm{F}}=33.8 \mathrm{~Hz},{ }^{4} \mathrm{~J}_{\mathrm{H}-\mathrm{F}}=2.8 \mathrm{~Hz}\right) .{ }^{13} \mathrm{C}-\mathrm{NMR}\left(75.5 \mathrm{MHz}, \mathrm{CDCl}_{3}\right) \delta 191.9(\mathrm{~d}$, $\left.{ }^{2} \mathrm{~J}_{\mathrm{C}-\mathrm{F}}=32 \mathrm{~Hz}, \mathrm{C}_{9}\right), 155.7\left(\mathrm{~d},{ }^{1} \mathrm{~J}_{\mathrm{C}-\mathrm{F}}=261 \mathrm{~Hz}, \mathrm{C}_{1}\right), 140.9\left(\mathrm{C}_{5}\right), 128.9\left(\mathrm{C}_{6 / 7}\right), 128.7$ $\left(\mathrm{C}_{6 / 7}\right), 126.7\left(\mathrm{C}_{8}\right), 119.0\left(\mathrm{~d},{ }^{2} \mathrm{~J}_{\mathrm{C}-\mathrm{F}}=13 \mathrm{~Hz}, \mathrm{C}_{2}\right), 34.8\left(\mathrm{~d},{ }^{4} \mathrm{~J}_{\mathrm{C}-\mathrm{F}}=2 \mathrm{~Hz}, \mathrm{C}_{4}\right), 26.3(\mathrm{~d}$, $\left.{ }^{3} \mathrm{~J}_{\mathrm{C}-\mathrm{F}}=3 \mathrm{~Hz}, \mathrm{C}_{3}\right), 25.9\left(\mathrm{C}_{10}\right) \cdot \mathrm{MS}(\mathrm{EI}) 192\left(\mathrm{M}^{+}\right), 172,91\left(\mathrm{PhCH}_{2}^{+}\right), 43\left(\mathrm{CH}_{3} \mathrm{CO}^{+}\right)$. IR (neat) 3063, 3027, 2929, 2862, 1713, 1693, 1651, 1496, 1454, 1361, 1287, 1099, 750, 700.Anal. Calcd for $\mathrm{C}_{12} \mathrm{H}_{13} \mathrm{FO}: \mathrm{C}, 74.98 ; \mathrm{H}, 6.82$. Found : C, 74.75; H, 6.85 .
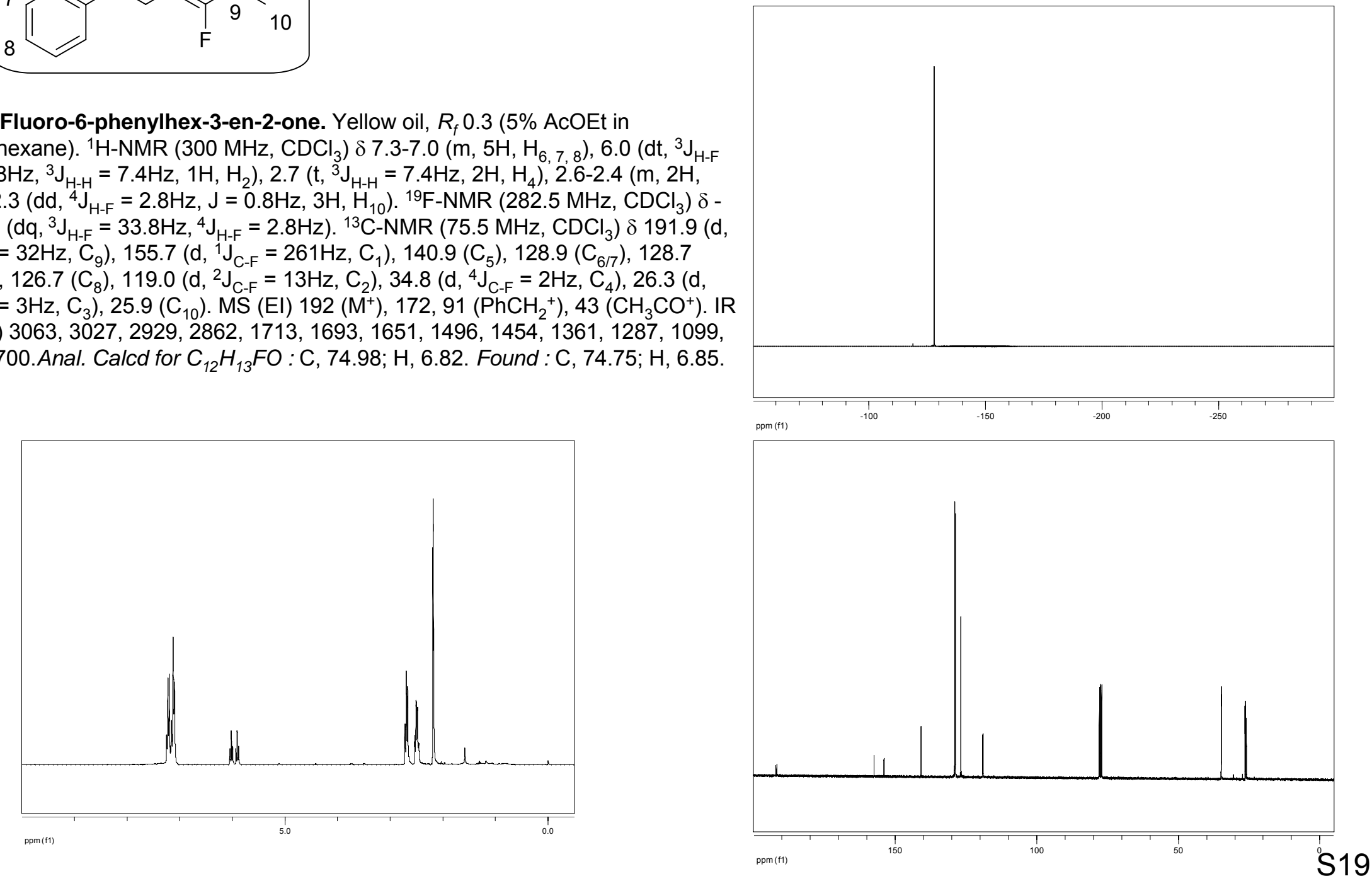


\section{Compound $\mathbf{2}_{\mathbf{8}}(\boldsymbol{Z})^{7 \mathrm{a}}$}



\section{Compound $\mathbf{4} \mathbf{a}_{\mathbf{8}}(\mathbf{Z})$}

(Z) 3-Fluoro-6-(oxytert-butyldiphenylsilyl)hex-3-en-2-one. Yellow oil, $R_{f} 0.2(2 \%$ AcOEt in cyclohexane). ${ }^{1} \mathrm{H}-\mathrm{NMR}\left(300 \mathrm{MHz}, \mathrm{CDCl}_{3}\right) \delta$ 7.7-7.5 (m, 4H, $\left.\mathrm{H}_{6}\right), 7.4-7.2$ $\left(\mathrm{m}, 6 \mathrm{H}, \mathrm{H}_{7,8}\right), 6.1\left(\mathrm{dt},{ }^{3} \mathrm{~J}_{\mathrm{H}-\mathrm{F}}=34.3 \mathrm{~Hz},{ }^{3} \mathrm{~J}_{\mathrm{H}-\mathrm{H}}=7.7 \mathrm{~Hz}, 1 \mathrm{H}, \mathrm{H}_{2}\right), 3.7\left(\mathrm{t},{ }^{3} \mathrm{~J}_{\mathrm{H}-\mathrm{H}}=6.4 \mathrm{~Hz}\right.$, $\left.2 \mathrm{H}, \mathrm{H}_{4}\right), 2.5-2.3\left(\mathrm{~m}, 2 \mathrm{H}, \mathrm{H}_{3}\right), 2.2\left(\mathrm{~d},{ }^{4} \mathrm{~J}_{\mathrm{H}-\mathrm{F}}=2.6 \mathrm{~Hz}, 3 \mathrm{H}, \mathrm{H}_{12}\right), 0.9\left(\mathrm{~s}, 9 \mathrm{H}, \mathrm{H}_{10}\right) \cdot{ }^{19} \mathrm{~F}-$ $\operatorname{NMR}\left(282.5 \mathrm{MHz}, \mathrm{CDCl}_{3}\right) \delta-127.4(\mathrm{~m}) .{ }^{13} \mathrm{C}-\mathrm{NMR}\left(75.5 \mathrm{MHz}, \mathrm{CDCl}_{3}\right) \delta 191.7\left(\mathrm{~d},{ }^{2} \mathrm{~J}_{\mathrm{C}}\right.$ $\left.\mathrm{F}=31 \mathrm{~Hz}, \mathrm{C}_{11}\right), 156.2\left(\mathrm{~d},{ }^{1} \mathrm{~J}_{\mathrm{C}-\mathrm{F}}=261 \mathrm{~Hz}, \mathrm{C}_{1}\right), 136.0\left(\mathrm{C}_{6}\right), 133.9\left(\mathrm{C}_{5}\right), 130.2\left(\mathrm{C}_{8}\right)$, $128.2\left(\mathrm{C}_{7}\right), 117.4\left(\mathrm{~d},{ }^{2} \mathrm{~J}_{\mathrm{C}-\mathrm{F}}=13 \mathrm{~Hz}, \mathrm{C}_{2}\right), 62.4\left(\mathrm{~d},{ }^{4} \mathrm{~J}_{\mathrm{C}-\mathrm{F}}=2 \mathrm{~Hz}, \mathrm{C}_{4}\right), 28.3\left(\mathrm{~d},{ }^{3} \mathrm{~J}_{\mathrm{C}-\mathrm{F}}=\right.$ $\left.3 \mathrm{~Hz}, \mathrm{C}_{3}\right), 27.2\left(\mathrm{C}_{10}\right), 25.9\left(\mathrm{C}_{12}\right), 19.6\left(\mathrm{C}_{9}\right)$. MS (EI) $313\left(\mathrm{M}^{+}\right.$-tBu $), 201,199,181,141$, $43\left(\mathrm{CH}_{3} \mathrm{CO}^{+}\right)$. IR (neat) 3410, 3072, 2932, 2858, 1722, 1697, 1471, 1428, 1362, 1114, 821, 701, 612, 509. Anal. Calcd for $\mathrm{C}_{22} \mathrm{H}_{27} \mathrm{FO}_{2} \mathrm{Si}$ : C, 71.31; H, 7.34. Found: C, $71.55 ; \mathrm{H}, 7.16$.



\section{of bromofluoroolefin:}

\section{With recovering of $(\mathrm{Z})$ starting bromofluoroolefin:}

To a mixture of potassium tert-butoxide (2 eq.) and freshly synthesized 1-(2-Ethoxyvinyl)benzene (2 eq.) in anhydrous THF $\left(5 \mathrm{~mL} / \mathrm{mmol}\right.$ of $t$-BuOK) at $-78^{\circ} \mathrm{C}$ under argon was added dropwise $n$-BuLi in hexanes $(1.6 \mathrm{M}, 2$ eq.). The mixture was stirred for $30 \mathrm{~min}$ at $-78^{\circ} \mathrm{C}$ and then a solution of dry zincchloride (4 eq.) in THF (4 mL/mmol of $\mathrm{ZnCl}_{2}$ ) was added dropwise. After $10 \mathrm{~min}$, the cooling bath was removed and the solution was allowed to warm to room temperature for $30 \mathrm{~min}$. The mixture was then added slowly to a solution of palladiumdiacetate (0.025 eq.), triphenylphosphine (0.05 eq.) and $(Z) \&(E)$ 1-(2-Bromo-2-fluorovinyl)-4-methoxybenzene (1 eq.) in anhydrous THF (10 mL/mmol of bromofluoroolein) at room temperature under argon. The mixture was stirred for $1 \mathrm{~h}$. After the coupling reaction was completed, controlled by monitoring ${ }^{19} \mathrm{~F}-\mathrm{NMR}$ signal of the reaction mixture, $\mathrm{HCl}$ aq. (6M) was added and the mixture was stirred and heated to $70^{\circ} \mathrm{C}$ for $1 \mathrm{~h}$. After cooling to room temperature, the mixture was then extracted with $\mathrm{Et}_{2} \mathrm{O}$ (x 3), washed with brine and the combined organic layers were dried over $\mathrm{MgSO}_{4}$. After filtration and concentration under reduced pressure, the residue was purified by chromatography on silica gel (eluent: $2 \%$ AcOEt in cyclohexane), affording (Z) 1-(2-Bromo-2-fluorovinyl)-4-methoxybenzene (yield = quant.) and (Z) 3Fluoro-4-(4-methoxyphenyl)-1-phenylbut-3-en-2one (yield = 93\%).

\section{With isomerization of $(E)$ enol ether-diene intermediate:}

To a mixture of potassium tert-butoxide (4 eq.), 1-(2-Ethoxyvinyl)benzene (4 eq.) in anhydrous THF (5 mL/mmol of $t$ $\mathrm{BuOK})$ at $-78^{\circ} \mathrm{C}$ under argon was added dropwise $n$-BuLi in hexanes (1.6M, 4 eq.). The mixture was stirred for $30 \mathrm{~min}$ at $-78^{\circ} \mathrm{C}$ and then a solution of dry zincchloride (8 eq.) in THF (4 mL/mmol of $\mathrm{ZnCl}_{2}$ ) was added dropwise. After 10 $\mathrm{min}$, the cooling bath was removed and the solution was allowed to warm to room temperature for $30 \mathrm{~min}$. The mixture was then added slowly to a solution of palladiumdiacetate (0.05 eq.), triphenylphosphine (0.1 eq.) and (Z) \& (E) 1-(2-Bromo-2-fluorovinyl)-4-methoxybenzene (1 eq.) in anhydrous THF (10 mL/mmol of bromofluoroolein) at $70^{\circ} \mathrm{C}$ under argon. The mixture was stirred for $1 \mathrm{~h}$. After the coupling reaction was completed, controlled by monitoring ${ }^{19} \mathrm{~F}-\mathrm{NMR}$ signal of the reaction mixture, $\mathrm{HCl}$ aq. $(6 \mathrm{M})$ was added and the mixture was stirred at $70^{\circ} \mathrm{C}$ for another hour. After cooling to room temperature, the mixture was then extracted with $\mathrm{Et}_{2} \mathrm{O}$ (x 3), washed with brine and the combined organic layers were dried over $\mathrm{MgSO}_{4}$. After filtration and concentration under reduced pressure, the residue was purified by chromatography on silica gel (eluent: 2\% AcOEt in cyclohexane), affording (Z) 3-Flugro4-(4-methoxyphenyl)-1-phenylbut-3-en-2one (yield = 99\%). 


\section{Compound $\mathbf{4} \mathbf{b}_{\mathbf{1}}(\mathbf{Z})$}

3-Fluoro-4-(4-methoxyphenyl)-1-phenylbut-3-en-2one. Yellow solid (m. p. = $\left.90-92^{\circ} \mathrm{C}\right), R_{f} 0.2$ ( $2 \%$ AcOEt in cyclohexane). ${ }^{1} \mathrm{H}-\mathrm{NMR}\left(300 \mathrm{MHz}, \mathrm{CDCl}_{3}\right) \delta 7.6$ (d, J $\left.=8.7 \mathrm{~Hz}, 2 \mathrm{H}, \mathrm{H}_{4}\right), 7.3-7.2\left(\mathrm{~m}, 5 \mathrm{H}, \mathrm{H}_{11,12.13}\right), 6.9\left(\mathrm{~d}, \mathrm{~J}=8.2 \mathrm{~Hz}, 2 \mathrm{H}, \mathrm{H}_{5}\right), 6.8\left(\mathrm{~d}, 3 \mathrm{~J}_{\mathrm{H}-\mathrm{F}}=\right.$ $\left.40.7 \mathrm{~Hz}, 1 \mathrm{H}, \mathrm{H}_{2}\right), 3.9\left(\mathrm{~d},{ }^{4} \mathrm{~J}_{\mathrm{H}-\mathrm{F}}=3.1 \mathrm{~Hz}, 2 \mathrm{H}, \mathrm{H}_{9}\right), 3.8\left(\mathrm{~s}, 3 \mathrm{H}, \mathrm{H}_{7}\right) .{ }^{19} \mathrm{~F}-\mathrm{NMR}(282.5$ $\left.\mathrm{MHz}, \mathrm{CDCl}_{3}\right) \delta-127.9\left(\mathrm{dt},{ }^{3} \mathrm{~J}_{\mathrm{H}-\mathrm{F}}=38.2 \mathrm{~Hz},{ }^{4} \mathrm{~J}_{\mathrm{H}-\mathrm{F}}=3.1 \mathrm{~Hz}\right) .{ }^{13} \mathrm{C}-\mathrm{NMR}(75.5 \mathrm{MHz}$, $\left.\mathrm{CDCl}_{3}\right) \delta 192.4\left(\mathrm{~d},{ }^{2} \mathrm{~J}_{\mathrm{C}-\mathrm{F}}=33 \mathrm{~Hz}, \mathrm{C}_{8}\right), 161.4\left(\mathrm{~d}, \mathrm{~J}=4 \mathrm{~Hz}, \mathrm{C}_{6}\right), 153.3\left(\mathrm{~d},{ }^{1} \mathrm{~J}_{\mathrm{CF}}=269 \mathrm{~Hz}\right.$ $\left.\mathrm{C}_{1}\right), 133.8\left(\mathrm{C}_{10}\right), 133.0\left(\mathrm{~d}, \mathrm{~J}=9 \mathrm{~Hz}, \mathrm{C}_{4}\right), 130.0\left(\mathrm{C}_{12}\right), 129.1\left(\mathrm{C}_{11}\right), 127.5\left(\mathrm{C}_{13}\right), 124.2$ $\left(\mathrm{d},{ }^{3} \mathrm{~J}_{\mathrm{C}-\mathrm{F}}=4 \mathrm{~Hz}, \mathrm{C}_{3}\right), 116.6\left(\mathrm{~d},{ }^{2} \mathrm{~J}_{\mathrm{C}-\mathrm{F}}=5 \mathrm{~Hz}, \mathrm{C}_{2}\right), 114.8\left(\mathrm{C}_{5}\right), 55.8\left(\mathrm{C}_{7}\right), 45.2\left(\mathrm{C}_{9}\right) . \mathrm{MS}$ (El) $270\left(\mathrm{M}^{+}\right), 179\left(\mathrm{M}^{+}-\mathrm{PhCH}_{2}\right), 151\left(\mathrm{M}^{+}-\mathrm{PhCH}_{2} \mathrm{CO}\right)$. IR (KBr) 3060, 3041, 2968, 2932, 2822, 1703, 1633, 1599, 1513, 1338, 1252, 1167, 1030, 837, 707, 533. Anal. Calcd for $\mathrm{C}_{17} \mathrm{H}_{15} \mathrm{FO}_{2}$ : C, 75.54; $\mathrm{H}, 5.59$. Found: $\mathrm{C}, 75.57 ; \mathrm{H}, 6.02$.



\section{Procedure for the obtention of $(Z)$ fluorinated benzylketones $4 b_{2}(Z)$ from mixture of bromofluoroolefins 2 .}

To a mixture of potassium tert-butoxide (2 eq.), freshly synthesized 1-(2-Ethoxyvinyl)benzene (2 eq.) in anhydrous THF ( $5 \mathrm{~mL} / \mathrm{mmol}$ of $t$-BuOK) at $-78^{\circ} \mathrm{C}$ under argon was added dropwise $n-\mathrm{BuLi}$ in hexanes (1.6M, 2 eq.). The mixture was stirred for $30 \mathrm{~min}$ at $-78^{\circ} \mathrm{C}$ and then a solution of dry zincchloride (4 eq.) in THF ( $4 \mathrm{~mL} / \mathrm{mmol}$ of $\mathrm{ZnCl}_{2}$ ) was added dropwise. After $10 \mathrm{~min}$, the cooling bath was removed and the solution was allowed to warm to room temperature for $30 \mathrm{~min}$. The mixture was then added slowly to a solution of palladiumdiacetate (0.025 eq.), triphenylphosphine (0.05 eq.) and mixture of bromofluoroolefins 2 (1 eq.) in anhydrous THF (10 mL/mmol of bromofluoroolefin) at $10^{\circ} \mathrm{C}$ under argon. The mixture was stirred for $1 \mathrm{~h}$. After the coupling reaction was completed, controlled by monitoring ${ }^{19} \mathrm{~F}-\mathrm{NMR}$ signal of the reaction mixture, $\mathrm{HCl}$ aq. $(1 \mathrm{~N})$ was added and the mixture was stirred at room temperature for $1 \mathrm{~h}$. The mixture was then extracted with $\mathrm{Et}_{2} \mathrm{O}(\mathrm{x} 3)$, washed with brine and the combined organic layers were dried over $\mathrm{MgSO}_{4}$. After filtration and concentration under reduced pressure, the residue was purified by chromatography on silica gel (eluent: 5\% AcOEt in cyclohexane), affording (Z) starting bromofluoroolefin $\mathbf{2 ( Z )}$ and $(Z)$ benzylketone $4 \mathrm{~b}(Z)$. 


\section{Compound $\mathbf{4} \mathbf{b}_{\mathbf{2}}(\mathbf{Z})$}

(Z) 3-Fluoro-1,6-diphenylhex-3-en-2-one. Orange oil, $R_{f} 0.4$ (5\% AcOEt in

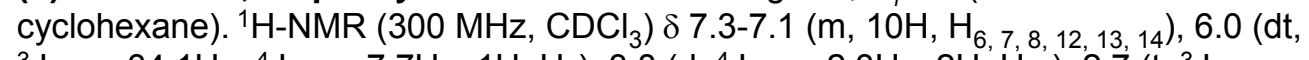
$\left.{ }^{3} \mathrm{~J}_{\mathrm{H}-\mathrm{F}}=34.1 \mathrm{~Hz},{ }^{4} \mathrm{~J}_{\mathrm{H}-\mathrm{F}}=7.7 \mathrm{~Hz}, 1 \mathrm{H}, \mathrm{H}_{2}\right), 3.8\left(\mathrm{~d},{ }^{4} \mathrm{~J}_{\mathrm{H}-\mathrm{F}}=2.3 \mathrm{~Hz}, 2 \mathrm{H}, \mathrm{H}_{10}\right), 2.7\left(\mathrm{t},{ }^{3} \mathrm{~J}_{\mathrm{H}-\mathrm{H}}=\right.$ $\left.7.5 \mathrm{~Hz}, 2 \mathrm{H}, \mathrm{H}_{4}\right), 2.5-2.4\left(\mathrm{~m}, 2 \mathrm{H}, \mathrm{H}_{3}\right) .{ }^{19} \mathrm{~F}-\mathrm{NMR}\left(282.5 \mathrm{MHz}, \mathrm{CDCl}_{3}\right) \delta-128.3\left(\mathrm{dt},{ }^{3} \mathrm{~J}_{\mathrm{H}-\mathrm{F}}=\right.$ $\left.34.4 \mathrm{~Hz},{ }^{4} \mathrm{~J}_{\mathrm{H}-\mathrm{F}}=2.3 \mathrm{~Hz}\right) .{ }^{13} \mathrm{C}-\mathrm{NMR}\left(75.5 \mathrm{MHz}, \mathrm{CDCl}_{3}\right) \delta 191.7\left(\mathrm{~d},{ }^{2} \mathrm{~J}_{\mathrm{C}-\mathrm{F}}=32 \mathrm{~Hz}, \mathrm{C}_{9}\right)$, $155.4\left(\mathrm{~d},{ }^{1} \mathrm{~J}_{\mathrm{C}-\mathrm{F}}=262 \mathrm{~Hz}, \mathrm{C}_{1}\right), 140.9\left(\mathrm{C}_{5}\right), 133.6\left(\mathrm{C}_{11}\right), 129.9\left(\mathrm{C}_{12 / 13}\right), 129.1\left(\mathrm{C}_{12 / 13}\right)$, $129.0\left(\mathrm{C}_{6 / 7}\right), 128.7\left(\mathrm{C}_{6 / 7}\right), 127.6\left(\mathrm{C}_{14}\right), 126.7\left(\mathrm{C}_{8}\right), 119.5\left(\mathrm{~d},{ }^{2} \mathrm{~J}_{\mathrm{C}-\mathrm{F}}=12 \mathrm{~Hz}, \mathrm{C}_{2}\right), 45.2$ $\left(\mathrm{C}_{10}\right), 34.8\left(\mathrm{C}_{4}\right), 26.5\left(\mathrm{C}_{3}\right)$. MS (EI) $268\left(\mathrm{M}^{+}\right), 177\left(\mathrm{M}^{+}-\mathrm{PhCH}_{2}\right), 157,91\left(\mathrm{PhCH}_{2}{ }^{+}\right), 65$. IR (neat) 3085, 3062, 3028, 2928, 2860, 1702, 1687, 1650, 1495, 1454, 1297, 1030, 748, 699. Anal. Calcd for $\mathrm{C}_{18} \mathrm{H}_{17}$ FO: C, 80.57; H, 6.39. Found: C, 80.69; H, 6.52.



\section{Procedure for the obtention of $(Z)$ fluorinated propylketones $4 c(Z)$ from mixture of bromofluoroolefins 2.}

To a mixture of potassium tert-butoxide (2 eq.), butenylethylether (2 eq.) in anhydrous THF ( $5 \mathrm{~mL} / \mathrm{mmol}$ of $t$-BuOK) at $-78^{\circ} \mathrm{C}$ under argon was added dropwise $n$-BuLi in hexanes $\left(1.6 \mathrm{M}, 2\right.$ eq.). The mixture was stirred for $30 \mathrm{~min}$ at $-78^{\circ} \mathrm{C}$ and then a solution of dry zincchloride (4 eq.) in THF ( $4 \mathrm{~mL} / \mathrm{mmol}$ of $\mathrm{ZnCl}_{2}$ ) was added dropwise. After $10 \mathrm{~min}$, the cooling bath was removed and the solution was allowed to warm to room temperature for $30 \mathrm{~min}$. The mixture was then added slowly to a solution of palladiumdiacetate (0.025 eq.), triphenylphosphine (0.05 eq.) and mixture of bromofluoroolefins 2 (1 eq.) in anhydrous THF $\left(10 \mathrm{~mL} / \mathrm{mmol}\right.$ of bromofluoroolefin) at $10^{\circ} \mathrm{C}$ under argon. The mixture was stirred for $1 \mathrm{~h}$. After the reaction was completed, controlled by monitoring ${ }^{19} \mathrm{~F}-\mathrm{NMR}$ signal of the reaction mixture, $\mathrm{HCl}$ aq. (1N) was added and the mixture was stirred for $15 \mathrm{~min}$. The mixture was then extracted with $\mathrm{Et}_{2} \mathrm{O}$ (x 3), washed with brine and the combined organic layers were dried over $\mathrm{MgSO}_{4}$. After filtration and concentration under reduced pressure, the residue was purified by chromatography on silica gel (eluent: cyclohexane/AcOEt), affording $(Z)$ starting bromofluoroolefin $\mathbf{2 ( Z )}$ and $(Z)$ propylketone $\mathbf{4 c}(Z)$. 


\section{Compound $\mathbf{4} \mathbf{c}_{1}(Z)$}

(Z) 2-Fluoro-1-(4-methoxyphenyl)hex-1-en-3-one. White solid (m. p. = 77-79 $\mathrm{C}$ ), $R_{f} 0.3\left(5 \%\right.$ AcOEt in cyclohexane). ${ }^{1} \mathrm{H}-\mathrm{NMR}\left(300 \mathrm{MHz}, \mathrm{CDCl}_{3}\right) \delta 7.6(\mathrm{~d}, \mathrm{~J}=9.0 \mathrm{~Hz}$, $\left.2 \mathrm{H}, \mathrm{H}_{4}\right), 6.9\left(\mathrm{~d}, \mathrm{~J}=9.0 \mathrm{~Hz}, 2 \mathrm{H}, \mathrm{H}_{5}\right), 6.8\left(\mathrm{~d},{ }^{3} \mathrm{~J}_{\mathrm{H}-\mathrm{F}}=37.3 \mathrm{~Hz}, 1 \mathrm{H}, \mathrm{H}_{2}\right), 3.8\left(\mathrm{~s}, 3 \mathrm{H}, \mathrm{H}_{7}\right)$, $2.7\left(\mathrm{dt},{ }^{4} \mathrm{~J}_{\mathrm{H}-\mathrm{F}}=2.3 \mathrm{~Hz},{ }^{3} \mathrm{~J}_{\mathrm{H}-\mathrm{H}}=7.5 \mathrm{~Hz}, 2 \mathrm{H}, \mathrm{H}_{\mathrm{g}}\right), 1.8-1.6\left(\mathrm{~m}, 2 \mathrm{H}, \mathrm{H}_{10}\right), 1.0\left(\mathrm{t},{ }^{3} \mathrm{~J}_{\mathrm{H}-\mathrm{H}}=\right.$ $\left.7.2 \mathrm{~Hz}, 3 \mathrm{H}, \mathrm{H}_{11}\right) .{ }^{19} \mathrm{~F}-\mathrm{NMR}\left(282.5 \mathrm{MHz}, \mathrm{CDCl}_{3}\right) \delta-128.6\left(\mathrm{~d},{ }^{3} \mathrm{~J}_{\mathrm{H}-\mathrm{F}}=36.7 \mathrm{~Hz}\right) .{ }^{13} \mathrm{C}-$ $\operatorname{NMR}\left(75.5 \mathrm{MHz}, \mathrm{CDCl}_{3}\right) \delta 195.2\left(\mathrm{~d},{ }^{2} \mathrm{~J}_{\mathrm{C}-\mathrm{F}}=31 \mathrm{~Hz}, \mathrm{C}_{8}\right), 161.2\left(\mathrm{C}_{6}\right), 153.6\left(\mathrm{~d},{ }^{1} \mathrm{~J}_{\mathrm{C}-\mathrm{F}}=\right.$ $\left.269 \mathrm{~Hz}, \mathrm{C}_{1}\right), 132.8\left(\mathrm{~d}, \mathrm{~J}=9 \mathrm{~Hz}, \mathrm{C}_{4}\right), 124.3\left(\mathrm{~d},{ }^{3} \mathrm{~J}_{\mathrm{C}-\mathrm{F}}=4 \mathrm{~Hz}, \mathrm{C}_{3}\right), 115.5\left(\mathrm{~d},{ }^{2} \mathrm{~J}_{\mathrm{CF}}=6 \mathrm{~Hz}\right.$ $\left.\mathrm{C}_{2}\right), 114.7\left(\mathrm{C}_{5}\right), 55.7\left(\mathrm{C}_{7}\right), 40.1\left(\mathrm{C}_{9}\right), 17.6\left(\mathrm{C}_{10}\right), 14.2\left(\mathrm{C}_{11}\right) \cdot \mathrm{MS}(\mathrm{EI}) 222\left(\mathrm{M}^{+}\right), 179$ $\left(\mathrm{M}^{+}-\mathrm{CH}_{3} \mathrm{CH}_{2} \mathrm{CH}_{2}\right), 152,136,108$. IR ( $\left.\mathrm{KBr}\right) 3019,2959,2936,2873,2848,1702$, $1637,1602,1508,1464,1381,1332,1250,1176,1037,840,543$. Anal. Calcd for $\mathrm{C}_{13} \mathrm{H}_{15} \mathrm{FO}_{2}: \mathrm{C}, 70.25 ; \mathrm{H}, 6.80$. Found: $\mathrm{C}, 69.85 ; \mathrm{H}, 6.71$.
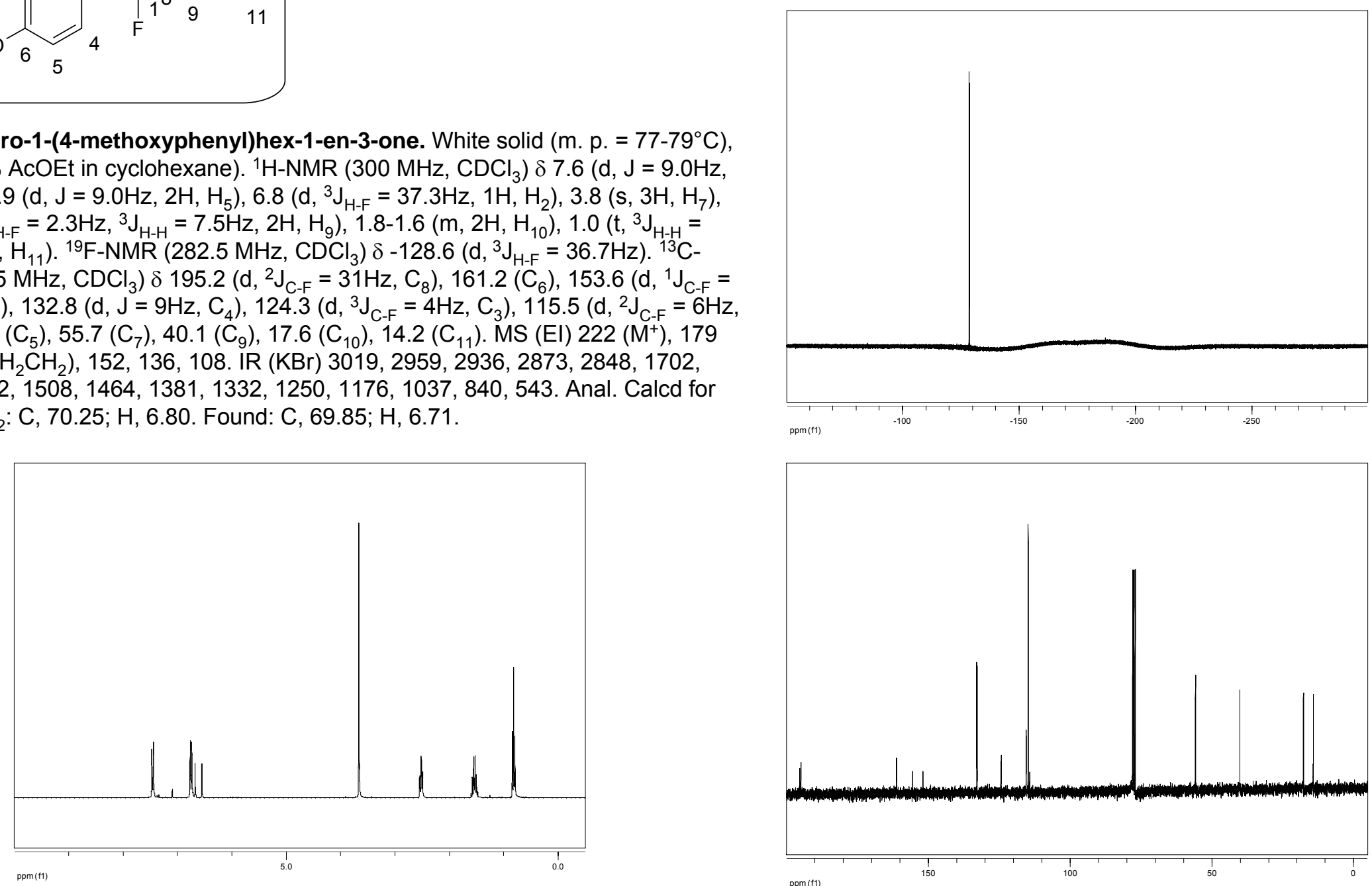


\section{Compound $\mathbf{4} \mathbf{c}_{2}(Z)$}

(Z) 4-Fluoro-1-phenyloct-3-en-5-one. Yellow oil, $R_{f} 0.5$ (5\% AcOEt in cyclohexane). ${ }^{1} \mathrm{H}-\mathrm{NMR}\left(300 \mathrm{MHz}, \mathrm{CDCl}_{3}\right) \delta 7.3-7.1\left(\mathrm{~m}, 5 \mathrm{H}, \mathrm{H}_{6,7,8}\right), 6.0\left(\mathrm{dt},{ }^{3} \mathrm{~J}_{\mathrm{H}-\mathrm{F}}=\right.$ $\left.34.3 \mathrm{~Hz},{ }^{3} \mathrm{~J}_{\mathrm{H}-\mathrm{H}}=7.7 \mathrm{~Hz}, 1 \mathrm{H}, \mathrm{H}_{2}\right), 2.7\left(\mathrm{~m}, 2 \mathrm{H}, \mathrm{H}_{4}\right), 2.5\left(\mathrm{~m}, 4 \mathrm{H}, \mathrm{H}_{3}, 10\right), 1.5\left(\mathrm{~h},{ }^{3} \mathrm{~J}_{\mathrm{H}-\mathrm{H}}=\right.$ $\left.7.4 \mathrm{~Hz}, 2 \mathrm{H}, \mathrm{H}_{11}\right), 0.9\left(\mathrm{t},{ }^{3} \mathrm{~J}_{\mathrm{H}-\mathrm{H}}=7.2 \mathrm{~Hz}, 3 \mathrm{H}, \mathrm{H}_{12}\right)$. ${ }^{19} \mathrm{~F}-\mathrm{NMR}\left(282.5 \mathrm{MHz}, \mathrm{CDCl}_{3}\right) \delta-$ $129.2\left(\mathrm{dt},{ }^{3} \mathrm{~J}_{\mathrm{H}-\mathrm{F}}=34.4 \mathrm{~Hz},{ }^{4} \mathrm{~J}_{\mathrm{H}-\mathrm{F}}=2.2 \mathrm{~Hz}\right) .{ }^{13} \mathrm{C}-\mathrm{NMR}\left(75.5 \mathrm{MHz}, \mathrm{CDCl}_{3}\right) \delta 194.2(\mathrm{~d}$ $\left.{ }^{2} \mathrm{~J}_{\mathrm{C}-\mathrm{F}}=31 \mathrm{~Hz}, \mathrm{C}_{9}\right), 155.4\left(\mathrm{~d},{ }^{1} \mathrm{~J}_{\mathrm{C}-\mathrm{F}}=262 \mathrm{~Hz}, \mathrm{C}_{1}\right), 140.6\left(\mathrm{C}_{5}\right), 128.6\left(\mathrm{C}_{6 / 7}\right), 128.4\left(\mathrm{C}_{6 / 7}\right)$, $126.4\left(\mathrm{C}_{8}\right), 117.8\left(\mathrm{~d},{ }^{2} \mathrm{~J}_{\mathrm{C}-\mathrm{F}}=12 \mathrm{~Hz}, \mathrm{C}_{2}\right), 39.8\left(\mathrm{C}_{10}\right), 34.6\left(\mathrm{C}_{4}\right), 26.0\left(\mathrm{C}_{3}\right), 17.2\left(\mathrm{C}_{11}\right)$, $13.8\left(\mathrm{C}_{12}\right)$. MS (EI) $220\left(\mathrm{M}^{+}\right), 157,128\left(\mathrm{M}^{+}-\mathrm{PhCH}_{3}\right), 91\left(\mathrm{PhCH}_{2}{ }^{+}\right)$. IR (neat) 3029, 2964, 2934, 2876, 1732, 1711, 1694, 1656, 1455, 1210, 1169, 995, 749, 700. Anal. Calcd for $\mathrm{C}_{14} \mathrm{H}_{17}$ FO: C, 76.33; $\mathrm{H}, 7.78$. Found: C, 76.27; $\mathrm{H}, 7.77$.

Procedure for the obtention of $(Z)$ fluorinated dihydrodioxine $\mathbf{7}(Z)$ from mixture of bromofluoroolefins $\mathbf{2}$. To 1,4diox-2-ene (0.85 eq.) in anhydrous THF ( $5 \mathrm{~mL} / \mathrm{mmol}$ of enol ether) at $-40^{\circ} \mathrm{C}$ under argon was added dropwise $t$-BuLi in hexanes (1.6M, 0.85 eq.). The mixture was stirred for $30 \mathrm{~min}$ at $-40^{\circ} \mathrm{C}$ and then a solution of dry zincchloride (1.7 eq.) in THF $\left(4 \mathrm{~mL} / \mathrm{mmol}\right.$ of $\mathrm{ZnCl}_{2}$ ) was added dropwise at $-78^{\circ} \mathrm{C}$. After $10 \mathrm{~min}$, the cooling bath was removed and the solution was allowed to warm to room temperature for $30 \mathrm{~min}$. The mixture was then added slowly to a solution of palladiumdiacetate ( 0.05 eq.), triphenylphosphine ( 0.1 eq.) and mixture of bromofluoroolefins 2 (1 eq.) in anhydrous THF $\left(10 \mathrm{~mL} / \mathrm{mmol}\right.$ of bromofluoroolefin) at $10^{\circ} \mathrm{C}$ under argon. The mixture was stirred for $1 \mathrm{~h}$. After the coupling reaction was completed, controlled by monitoring ${ }^{19} \mathrm{~F}-\mathrm{NMR}$ signal of the reaction mixture, $\mathrm{NH}_{4} \mathrm{Cl}$ aq. (sat.) was added and the mixture was stirred for $5 \mathrm{~min}$. The mixture was then extracted with $\mathrm{Et}_{2} \mathrm{O}$ (x 3), washed with brine and the combined organic layers were dried over $\mathrm{MgSO}_{4}$. After filtration and concentration under reduced pressure, the residue was purified by chromatography on basic alumina (eluent: cyclohexane), affording $(Z)$ starting bromofluoroolefin $\mathbf{2 ( Z )}$ and $(Z)$ fluorinated dihydrodioxine $\mathbf{7 ( Z )}$. 


\section{Compound $\mathbf{7}_{1}(Z)$}

(Z) 5-(1-Fluoro-2-(4-methoxyphenyl)vinyl)-2,3-dihydro-1,4-dioxene. White solid (m. p. $\left.=111-113^{\circ} \mathrm{C}\right), R_{f} 0.3$ (cyclohexane, Aluminium oxide neutral). ${ }^{1} \mathrm{H}-\mathrm{NMR}(300$ $\left.\mathrm{MHz}, \mathrm{CDCl}_{3}\right) \delta 7.4\left(\mathrm{~d}, \mathrm{~J}=8.7 \mathrm{~Hz}, 2 \mathrm{H}, \mathrm{H}_{4}\right), 6.8\left(\mathrm{~d}, \mathrm{~J}=8.6 \mathrm{~Hz}, 2 \mathrm{H}, \mathrm{H}_{5}\right), 6.5\left(\mathrm{~d},{ }^{4} \mathrm{~J}_{\mathrm{H}-\mathrm{F}}=\right.$ $\left.2.6 \mathrm{~Hz}, 1 \mathrm{H}, \mathrm{H}_{\mathrm{g}}\right), 5.7\left(\mathrm{~d},{ }^{3} \mathrm{~J}_{\mathrm{H}-\mathrm{F}}=41.5 \mathrm{~Hz}, 1 \mathrm{H}, \mathrm{H}_{2}\right), 4.1-4.0\left(\mathrm{~m}, 4 \mathrm{H}, \mathrm{H}_{10}, 11\right), 3.7(\mathrm{~s}, 3 \mathrm{H}$, $\left.\mathrm{H}_{7}\right) .{ }^{19} \mathrm{~F}-\mathrm{NMR}\left(282.5 \mathrm{MHz}, \mathrm{CDCl}_{3}\right) \delta-133.8\left(\mathrm{dd},{ }^{3} \mathrm{~J}_{\mathrm{H}-\mathrm{F}}=41.3 \mathrm{~Hz},{ }^{4} \mathrm{~J}_{\mathrm{H}-\mathrm{F}}=2.3 \mathrm{~Hz}\right) .{ }^{13} \mathrm{C}-$ $\operatorname{NMR}\left(75.5 \mathrm{MHz}, \mathrm{CDCl}_{3}\right) \delta 158.8\left(\mathrm{C}_{6}\right), 150.8\left(\mathrm{~d},{ }^{1} \mathrm{~J}_{\mathrm{C}-\mathrm{F}}=249 \mathrm{~Hz}, \mathrm{C}_{1}\right), 129.8\left(\mathrm{~d},{ }^{2} \mathrm{~J}_{\mathrm{C}-\mathrm{F}}\right.$ $\left.=36 \mathrm{~Hz}, \mathrm{C}_{8}\right), 130.3\left(\mathrm{~d}, \mathrm{~J}=7 \mathrm{~Hz}, \mathrm{C}_{4}\right), 126.7\left(\mathrm{~d},{ }^{3} \mathrm{~J}_{\mathrm{C}-\mathrm{F}}=7 \mathrm{~Hz}, \mathrm{C}_{9}\right), 125.3\left(\mathrm{C}_{3}\right), 114.3$ $\left(\mathrm{C}_{5}\right), 102.2\left(\mathrm{~d},{ }^{2} \mathrm{~J}_{\mathrm{CF}}=6 \mathrm{~Hz}, \mathrm{C}_{2}\right), 65.0\left(\mathrm{C}_{1011}\right), 64.7\left(\mathrm{C}_{10011}\right), 55.6\left(\mathrm{C}_{7}\right) . \mathrm{MS}(\mathrm{EI}) 236$ $\left(\mathrm{M}^{+}\right), 179\left(\mathrm{M}^{+}-\mathrm{CH}_{2}-\mathrm{O}-\mathrm{CH}=\mathrm{CH}_{2}\right), 159,108\left(\mathrm{PhOCH}_{3}{ }^{+}\right)$. IR $(\mathrm{KBr}) 3115,2941,2842$, 1645, 1643, 1608, 1511, 1315, 1288, 1254, 1165, 1098, 1031, 919, 860, 816, 799, 535. Anal. Calcd for $\mathrm{C}_{13} \mathrm{H}_{13} \mathrm{FO}_{3}: \mathrm{C}, 66.09 ; \mathrm{H}, 5.55$. Found: $\mathrm{C}, 66.14 ; \mathrm{H}, 5.62$.
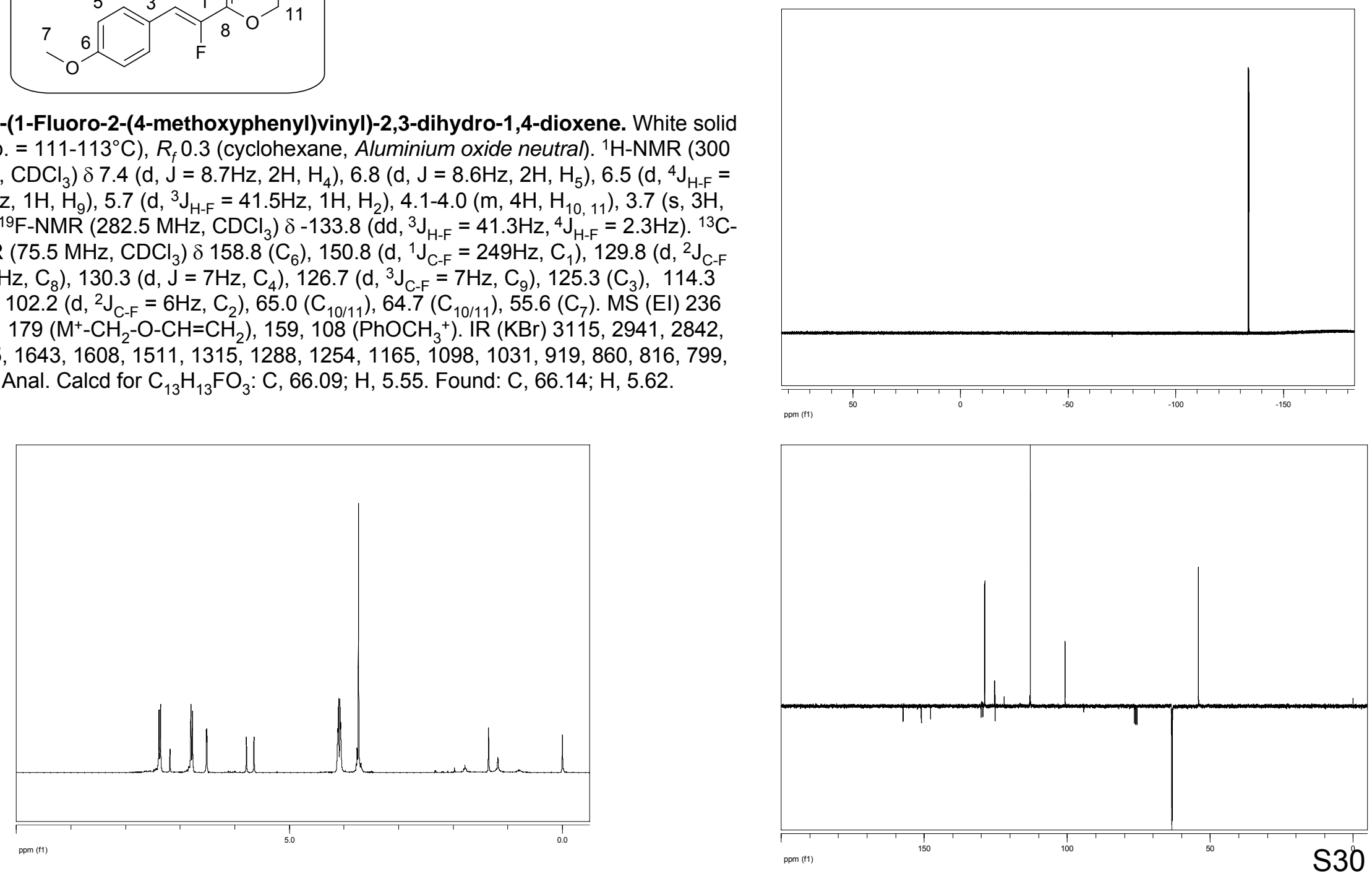


\section{Compound $\mathbf{7}_{\mathbf{2}}(\mathrm{Z})$}

(Z) 5-(1-Fluoro-4-phenylbut-1-enyl)-2,3-dihydro-1,4-dioxene. Yellow solid (m. p. $=73-75^{\circ} \mathrm{C}$ ), $R_{f} 0.3$ (cyclohexane, Aluminium oxide neutral). ${ }^{1} \mathrm{H}-\mathrm{NMR}(300 \mathrm{MHz}$, $\left.\mathrm{CDCl}_{3}\right) \delta 7.3-7.1\left(\mathrm{~m}, 5 \mathrm{H}, \mathrm{H}_{6,7.8}\right), 6.4\left(\mathrm{~d},{ }^{4} \mathrm{~J}_{\mathrm{H}-\mathrm{F}}=2.5 \mathrm{~Hz}, 1 \mathrm{H}, \mathrm{H}_{10}\right), 4.9\left(\mathrm{dt},{ }^{3} \mathrm{~J}_{\mathrm{H}-\mathrm{F}}=\right.$ $\left.38.7 \mathrm{~Hz},{ }^{3} \mathrm{~J}_{\mathrm{H}-\mathrm{H}}=7.7 \mathrm{~Hz}, 1 \mathrm{H}, \mathrm{H}_{2}\right), 4.0\left(\mathrm{~m}, 4 \mathrm{H}, \mathrm{H}_{11} 12\right), 2.6\left(\mathrm{~m}, 2 \mathrm{H}, \mathrm{H}_{4}\right), 2.4(\mathrm{~m}, 2 \mathrm{H}$ $\left.\mathrm{H}_{3}\right) .{ }^{19} \mathrm{~F}-\mathrm{NMR}\left(282.5 \mathrm{MHz}, \mathrm{CDCl}_{3}\right) \delta-135.6\left(\mathrm{~d},{ }^{3} \mathrm{~J}_{\mathrm{H}-\mathrm{F}}=38.7 \mathrm{~Hz}\right) .{ }^{13} \mathrm{C}-\mathrm{NMR}(75.5$ $\left.\mathrm{MHz}, \mathrm{CDCl}_{3}\right) \delta 151.4\left(\mathrm{~d},{ }^{1} \mathrm{~J}_{\mathrm{C}-\mathrm{F}}=240 \mathrm{~Hz}, \mathrm{C}_{1}\right), 141.7\left(\mathrm{C}_{5}\right), 130.6\left(\mathrm{~d},{ }^{3} \mathrm{~J}_{\mathrm{C}-\mathrm{F}}=20 \mathrm{~Hz}\right.$, $\left.\mathrm{C}_{10}\right), 129.5\left(\mathrm{~d},{ }^{2} \mathrm{~J}_{\mathrm{C}-\mathrm{F}}=38 \mathrm{~Hz}, \mathrm{C}_{9}\right), 128.8\left(\mathrm{C}_{6 / 7}\right), 126.3\left(\mathrm{C}_{6 / 7}\right), 126.1\left(\mathrm{C}_{8}\right), 102.1(\mathrm{~d}$, $\left.{ }^{2} \mathrm{~J}_{\mathrm{C}-\mathrm{F}}=13 \mathrm{~Hz}, \mathrm{C}_{2}\right), 64.6\left(\mathrm{C}_{11 / 12}\right), 64.4\left(\mathrm{C}_{11 / 12}\right), 35.8\left(\mathrm{~d},{ }^{4} \mathrm{~J}_{\mathrm{C}-\mathrm{F}}=1 \mathrm{~Hz}, \mathrm{C}_{4}\right), 25.4\left(\mathrm{~d},{ }^{3} \mathrm{~J}_{\mathrm{C}-\mathrm{F}}\right.$ $\left.=5 \mathrm{~Hz}, \mathrm{C}_{3}\right) \cdot \mathrm{MS}(\mathrm{EI}) 234\left(\mathrm{M}^{+}\right), 214,157\left(\mathrm{M}^{+}-\mathrm{Ph}\right), 143\left(\mathrm{M}^{+}-\mathrm{CH}_{2} \mathrm{Ph}\right), 128,99,91$ $\left(\mathrm{PhCH}_{2}{ }^{+}\right)$, 51. IR (KBr) 3113, 3025, 2930, 2862, 1687, 1649, 1453, 1313, 1281, $1163,1099,1080,1036,920,815,752,699$. Anal. Calcd for $\mathrm{C}_{14} \mathrm{H}_{15} \mathrm{FO}_{2}: \mathrm{C}, 71.78$ $H, 6.45$. Found: $C, 71.83 ; H, 6.41$.
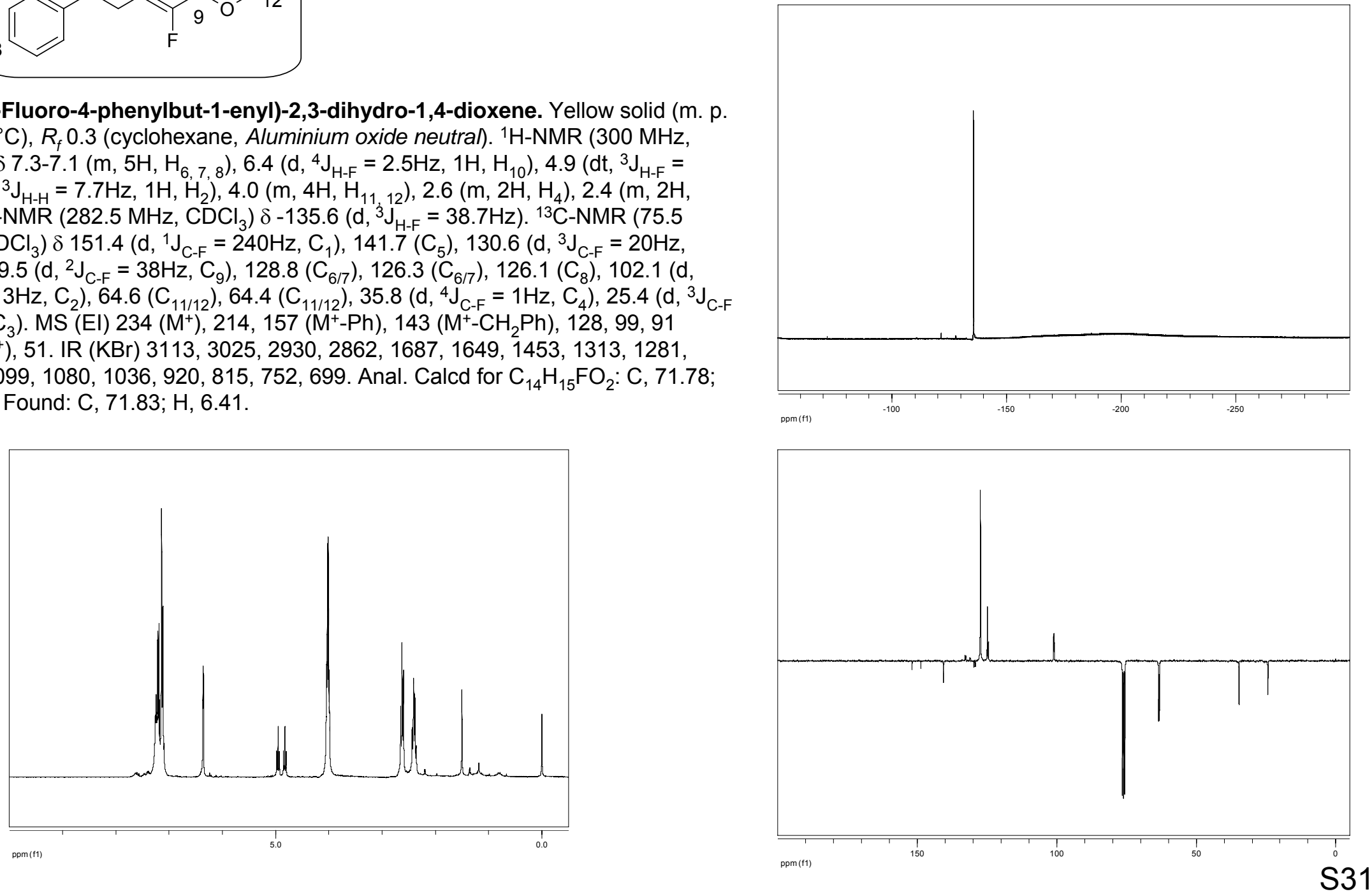
Procedure for the obtention of 3-Fluoro-1-hydroxy-4(4-methoxyphenyl)but-3-en-2-one 4d. Representative example $\mathbf{4 d ( Z ) : ~ T o ~ a ~ s o l u t i o n ~ o f ~ 5 - ( 1 - F l u o r o - 2 - ( 4 - m e t h o x y p h e n y l ) v i n y l ) - 2 , 3 - d i h y d r o - 1 , 4 - d i o x e n e ~ 7 ( Z ) - 1 3 ~ i n ~ m e t h a n o l ~ ( 5 0 ~}$ $\mathrm{mL} / \mathrm{mmol}$ of diene) and $\mathrm{CH}_{2} \mathrm{Cl}_{2}\left(25 \mathrm{~mL} / \mathrm{mmol}\right.$ of diene) at $0^{\circ} \mathrm{C}$ was added dropwise a solution of mCPBA (2 eq.) in $\mathrm{MeOH}\left(12 \mathrm{~mL} / \mathrm{mmol}\right.$ of diene). The solution was stirred at $0^{\circ} \mathrm{C}$ for $30 \mathrm{~min}$ and then neutralized with $\mathrm{NaHCO}_{3}$ aq. (5\%). Solvents were removed under reduced pressure and the product was extracted with $\mathrm{CH}_{2} \mathrm{Cl}_{2}$ (x 6). The combined organic layers were dried over $\mathrm{MgSO}_{4}$. After filtration and concentration under reduced pressure, the residue was taken up in EtOH $\left(75 \mathrm{~mL} / \mathrm{mmol}\right.$ of diene) and $\mathrm{NaBH}_{4}\left(1.32 \mathrm{eq}\right.$.) was added in three portions at $0^{\circ} \mathrm{C}$. The mixture was stirred at room temperature for $5 \mathrm{~h}$ and $\mathrm{EtOH}$ was removed. The residue was then diluted in $\mathrm{CH}_{2} \mathrm{Cl}_{2}$ and water was added. After extraction, the organic layer was dried over $\mathrm{MgSO}_{4}$. After filtration and concentration under reduced pressure, the crude diol was taken up in $1 \%$ aq. Acetone ( $50 \mathrm{~mL} / \mathrm{mmol}$ of diene) and PTSa was added ( 0.13 eq.). The solution was heated at $55^{\circ} \mathrm{C}$ for $2 \mathrm{~h}$ and then neutralized with $\mathrm{NaHCO}_{3}$ aq. (sat.). The mixture was extracted with AcOEt (3x) and dried over $\mathrm{MgSO}_{4}$. After filtration and concentration under reduced pressure, the residue was purified by chromatography on silica gel (eluent: 33\% AcOEt in cyclohexane), affording the desired alkoxyketone $\mathbf{4 d}(\mathbf{Z})$. 


\section{Compound $4 \mathbf{d}_{1}(Z)$}

Z) 3-Fluoro-1-hydroxy-4(4-methoxyphenyl)but-3-en-2-one. Yellow solid (m. p. $\left.=118-120^{\circ} \mathrm{C}\right), R_{f} 0.3\left(33 \%\right.$ AcOEt in cyclohexane). ${ }^{1} \mathrm{H}-\mathrm{NMR}\left(300 \mathrm{MHz}, \mathrm{CDCl}_{3}\right)$ $\delta 7.6\left(\mathrm{~d}, \mathrm{~J}=9.0 \mathrm{~Hz}, 2 \mathrm{H}, \mathrm{H}_{4}\right), 6.9\left(\mathrm{~d}, \mathrm{~J}=9.0 \mathrm{~Hz}, 2 \mathrm{H}, \mathrm{H}_{5}\right), 6.8\left(\mathrm{~d},{ }^{3} \mathrm{~J}_{\mathrm{H}-\mathrm{F}}=37.7 \mathrm{~Hz}, 1 \mathrm{H}\right.$, $\left.\mathrm{H}_{2}\right), 4.6\left(\mathrm{~d},{ }^{4} \mathrm{~J}_{\mathrm{H}-\mathrm{F}}=4.1 \mathrm{~Hz}, 2 \mathrm{H}, \mathrm{H}_{9}\right), 3.8\left(\mathrm{~s}, 3 \mathrm{H}, \mathrm{H}_{7}\right), 3.1(\mathrm{~b}, 1 \mathrm{H}, \mathrm{OH}) .{ }^{19} \mathrm{~F}-\mathrm{NMR}$ $\left(282.5 \mathrm{MHz}, \mathrm{CDCl}_{3}\right) \delta-135.6\left(\mathrm{~d},{ }^{3} \mathrm{~J}_{\mathrm{H}-\mathrm{F}}=37.9 \mathrm{~Hz}\right) .{ }^{13} \mathrm{C}-\mathrm{NMR}\left(75.5 \mathrm{MHz}, \mathrm{CDCl}_{3}\right) \delta$ $193.8\left(\mathrm{~d},{ }^{2} \mathrm{~J}_{\mathrm{C}-\mathrm{F}}=36 \mathrm{~Hz}, \mathrm{C}_{8}\right), 161.8\left(\mathrm{~d}, \mathrm{~J}=4 \mathrm{~Hz} \mathrm{C}_{6}\right), 151.6\left(\mathrm{~d},{ }^{1} \mathrm{~J}_{\mathrm{C}-\mathrm{F}}=264 \mathrm{~Hz}, \mathrm{C}_{1}\right)$, $133.4\left(\mathrm{~d}, \mathrm{~J}=9 \mathrm{~Hz} \mathrm{C}_{4}\right), 123.5\left(\mathrm{~d}, \mathrm{~J}=4 \mathrm{~Hz}, \mathrm{C}_{3}\right), 117.3\left(\mathrm{~d},{ }^{2} \mathrm{~J}_{\mathrm{C}-\mathrm{F}}=4 \mathrm{~Hz}, \mathrm{C}_{2}\right), 114.9$ $\left(\mathrm{C}_{5}\right), 65.8\left(\mathrm{C}_{9}\right), 55.8\left(\mathrm{C}_{7}\right)$. MS (EI) $210\left(\mathrm{M}^{+}\right), 194,179\left(\mathrm{M}^{+}-\mathrm{OCH}_{3}\right), 161\left(\mathrm{M}^{+}-\right.$ $\left.\mathrm{OCH}_{3} \& \mathrm{H}_{2} \mathrm{O}\right)$. IR (KBr) 3440, 3380, 3105, 2966, 1712, 1644, 1604, 1514, 1300, $1260,1239,1170,1100,1030,999,830$. Anal. Calcd for $\mathrm{C}_{11} \mathrm{H}_{11} \mathrm{FO}_{3}: \mathrm{C}, 62.85 ; \mathrm{H}$, 5.27. Found: C, 62.62; H, 5.34 .
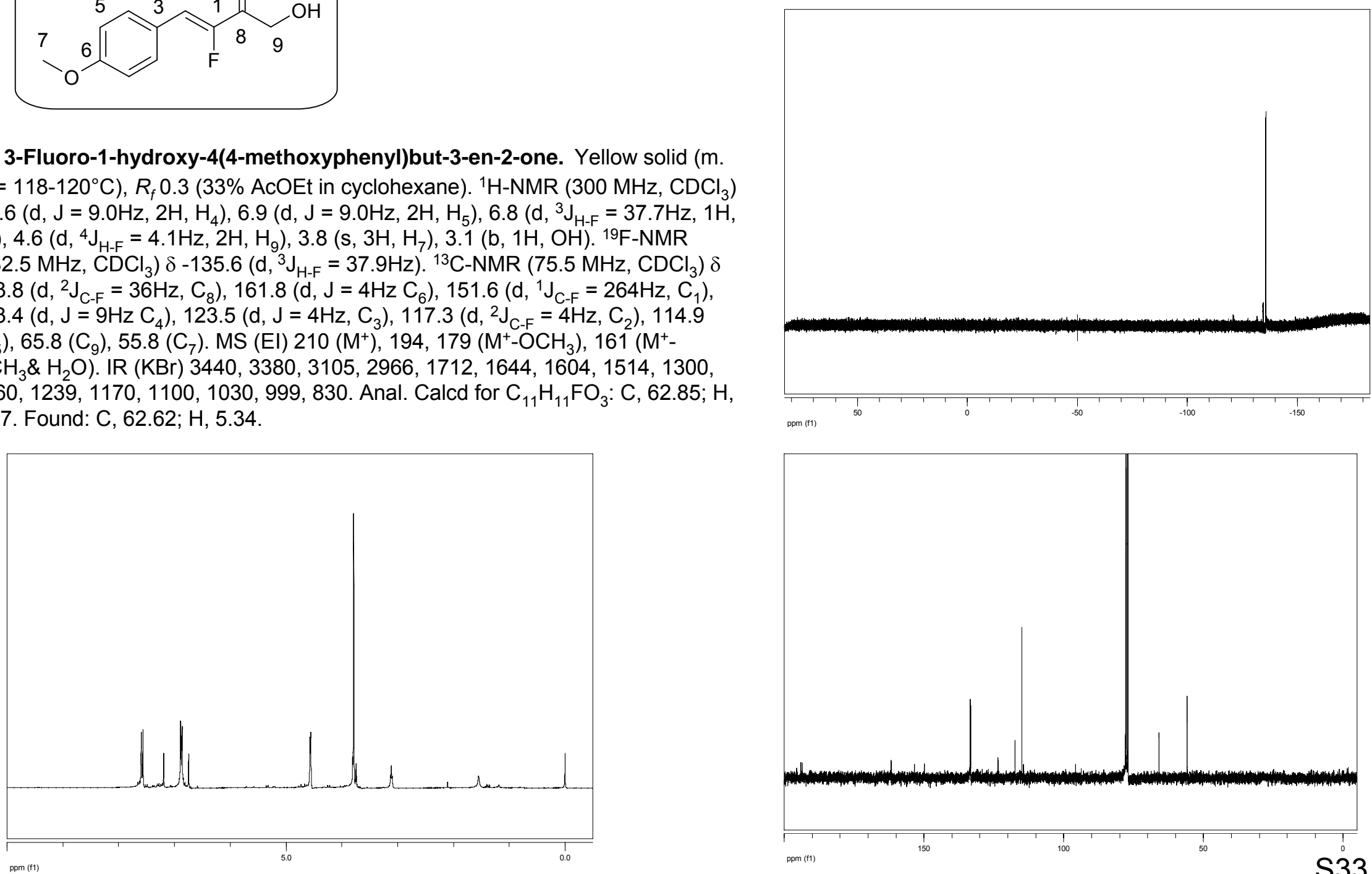

pom (11)

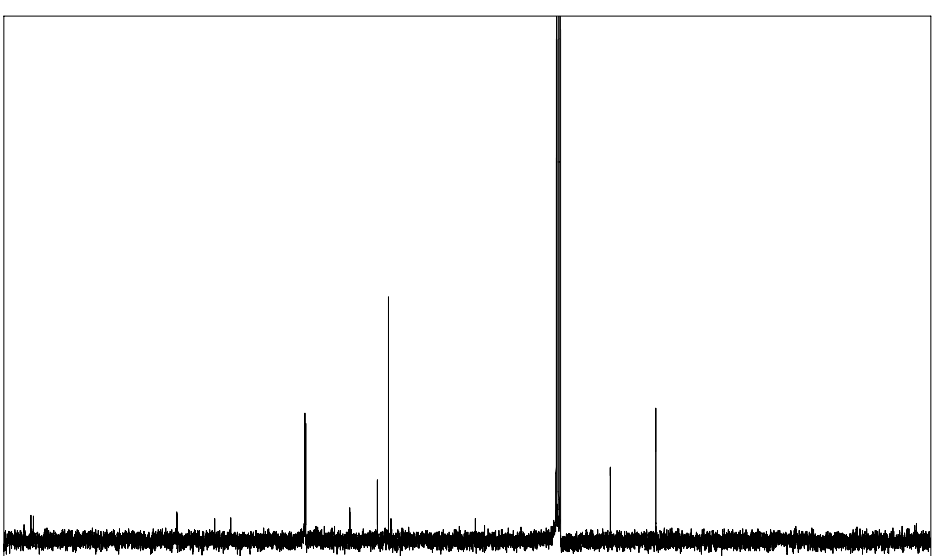

ppm(11)

150

${ }_{100}^{1}$ 
Procedure for the obtention of $(E)$ fluorinated methylketones $4 a(E)$ from $(Z)$ bromofluoroolefins. To a mixture of potassium tert-butoxide (2 eq.), ethylvinylether (2 eq.) in anhydrous THF ( $5 \mathrm{~mL} / \mathrm{mmol}$ of $t$-BuOK) at $-78^{\circ} \mathrm{C}$ under argon was added dropwise $n$-BuLi in hexanes (1.6M, 2 eq.). The mixture was stirred for $30 \mathrm{~min}$ at $-78^{\circ} \mathrm{C}$ and then a solution of dry zincchloride (4 eq.) in THF ( $4 \mathrm{~mL} / \mathrm{mmol}$ of $\mathrm{ZnCl}_{2}$ ) was added dropwise. After $10 \mathrm{~min}$, the cooling bath was removed and the solution was allowed to warm to room temperature for $30 \mathrm{~min}$. The mixture was then added slowly to a solution of palladiumdiacetate (0.05 eq.), triphenylphosphine (0.1 eq.) and bromofluoroolefin 2(Z) (1 eq.) in anhydrous THF (10 mL/mmol of bromofluoroolefin) at $70^{\circ} \mathrm{C}$ under argon. The mixture was stirred for $1 \mathrm{~h}$. After the reaction was completed, controlled by monitoring ${ }^{19} \mathrm{~F}-\mathrm{NMR}$ signal of the reaction mixture, the solution was allowed to cool to room temperature. $\mathrm{HCl}$ aq. $(1 \mathrm{~N})$ was added and the mixture was stirred for $15 \mathrm{~min}$. The mixture was then extracted with $\mathrm{Et}_{2} \mathrm{O}$ (x 3), washed with brine and the combined organic layers were dried over $\mathrm{MgSO}_{4}$. After filtration and concentration under reduced pressure at low temperature, the residue was purified by chromatography on silica gel (eluent: cyclohexane/AcOEt), affording $(E)$ methylketone $\mathbf{4 a}(E)$. 


\section{Compound $\mathbf{4 a} \mathbf{a}_{1}(\boldsymbol{E})$}

(E) 3-Fluoro-4-(4-methoxyphenyl)but-3-en-2-one. Yellow solid (m. p. $\left.<44^{\circ} \mathrm{C}\right), R_{f}$ $0.2\left(5 \%\right.$ AcOEt in cyclohexane). ${ }^{1} \mathrm{H}-\mathrm{NMR}\left(300 \mathrm{MHz}, \mathrm{CDCl}_{3}\right) \delta 7.6(\mathrm{~d}, \mathrm{~J}=8.9 \mathrm{~Hz}, 2 \mathrm{H}$, $\left.\mathrm{H}_{4}\right), 6.9\left(\mathrm{~d}, \mathrm{~J}=8.9 \mathrm{~Hz}, 2 \mathrm{H}, \mathrm{H}_{5}\right), 6.7\left(\mathrm{~d},{ }^{3} \mathrm{~J}_{\mathrm{H}-\mathrm{F}}=26.1 \mathrm{~Hz}, 1 \mathrm{H}, \mathrm{H}_{2}\right), 3.7\left(\mathrm{~s}, 3 \mathrm{H}, \mathrm{H}_{7}\right), 2.3(\mathrm{~d}$, $\left.{ }^{4} \mathrm{~J}_{\mathrm{H}-\mathrm{F}}=5.1 \mathrm{~Hz}, 3 \mathrm{H}, \mathrm{H}_{9}\right) .{ }^{19} \mathrm{~F}-\mathrm{NMR}\left(282.5 \mathrm{MHz}, \mathrm{CDCl}_{3}\right) \delta-115.2\left(\mathrm{~d},{ }^{3} \mathrm{~J}_{\mathrm{H}-\mathrm{F}}=26.1 \mathrm{~Hz},{ }^{4} \mathrm{~J}_{\mathrm{H}-}\right.$ $\mathrm{F}=5.1 \mathrm{~Hz}) \cdot{ }^{13} \mathrm{C}-\mathrm{NMR}\left(75.5 \mathrm{MHz}, \mathrm{CDCl}_{3}\right) \delta 193.4\left(\mathrm{~d},{ }^{2} \mathrm{~J}_{\mathrm{C}-\mathrm{F}}=39 \mathrm{~Hz}, \mathrm{C}_{8}\right), 161.2(\mathrm{~d}, \mathrm{~J}=$ $\left.2 \mathrm{~Hz}, \mathrm{C}_{6}\right), 152.6\left(\mathrm{~d},{ }^{1} \mathrm{~J}_{\mathrm{C}-\mathrm{F}}=252 \mathrm{~Hz}, \mathrm{C}_{1}\right), 132.6\left(\mathrm{~d}, \mathrm{~J}=3 \mathrm{~Hz}, \mathrm{C}_{4}\right), 123.5\left(\mathrm{~d},{ }^{3} \mathrm{~J}_{\mathrm{C}-\mathrm{F}}=10 \mathrm{~Hz}\right.$, $\left.\mathrm{C}_{3}\right), 121.0\left(\mathrm{~d},{ }^{2} \mathrm{~J}_{\mathrm{C}-\mathrm{F}}=29 \mathrm{~Hz}, \mathrm{C}_{2}\right), 114.1\left(\mathrm{C}_{5}\right), 55.7\left(\mathrm{C}_{7}\right), 28.6\left(\mathrm{~d},{ }^{3} \mathrm{~J}_{\mathrm{C}-\mathrm{F}}=4 \mathrm{~Hz}, \mathrm{C}_{9}\right) . \mathrm{MS}$ (El) $194\left(\mathrm{M}^{+}\right), 179\left(\mathrm{M}^{+}-\mathrm{CH}_{3}\right), 159,43\left(\mathrm{CH}_{3} \mathrm{CO}^{+}\right)$. IR $(\mathrm{KBr}) 3005,2937,2839,1729$, 1681, 1601, 1513, 1462, 1423, 1371, 1304, 1257, 1177, 1031, 832, 599, 533.Anal. Calcd for $\mathrm{C}_{11} \mathrm{H}_{11} \mathrm{FO}_{2}$ : C, 68.03; $\mathrm{H}, 5.71$. Found : C, 68.09; $\mathrm{H}, 5.54$.
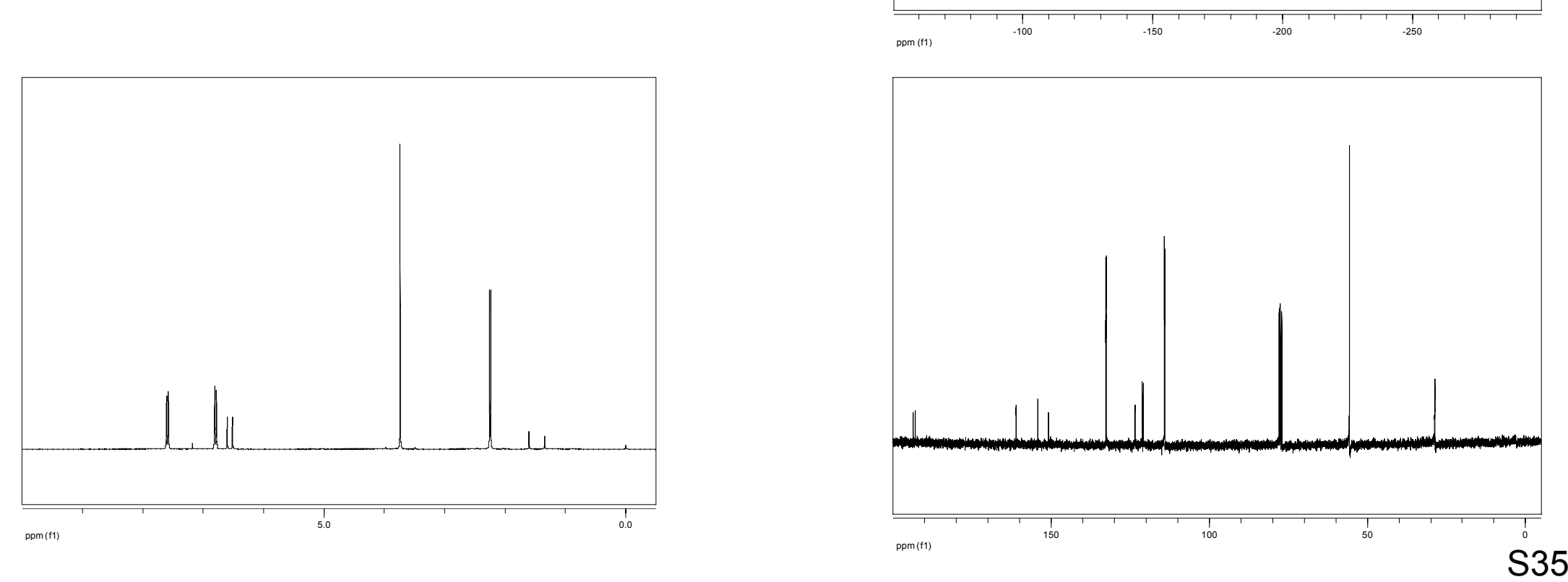


\section{Compound $\mathbf{4} \mathbf{a}_{2}(E)$}

(E) 3-Fluoro-4-(naphthalen-7-yl)but-3-en-2-one. Yellow solid (m. p. $\left.=62-64^{\circ} \mathrm{C}\right), R_{f}$ 0.3 (5\% AcOEt in cyclohexane). ${ }^{1} \mathrm{H}-\mathrm{NMR}\left(300 \mathrm{MHz}, \mathrm{CDCl}_{3}\right) \delta 8.1\left(\mathrm{~s}, 1 \mathrm{H}, \mathrm{H}_{12}\right), 7.9-$ $7.6\left(\mathrm{~m}, 3 \mathrm{H}, \mathrm{H}_{7,8,9}\right), 7.8-7.6\left(\mathrm{~m}, 1 \mathrm{H}, \mathrm{H}_{10}\right), 7.6-7.4\left(\mathrm{~m}, 2 \mathrm{H}, \mathrm{H}_{4,5}\right), 6.9\left(\mathrm{~d},{ }^{3} \mathrm{~J}_{\mathrm{H}-\mathrm{F}}=\right.$ $\left.24.6 \mathrm{~Hz}, 1 \mathrm{H}, \mathrm{H}_{2}\right), 2.4\left(\mathrm{~d},{ }^{4} \mathrm{~J}_{\mathrm{H}-\mathrm{F}}=4.9 \mathrm{~Hz}, 3 \mathrm{H}, \mathrm{H}_{14}\right) .{ }^{19} \mathrm{~F}-\mathrm{NMR}\left(282.5 \mathrm{MHz}, \mathrm{CDCl}_{3}\right) \delta-$ $112.8\left(\mathrm{dq},{ }^{3} \mathrm{~J}_{\mathrm{H}-\mathrm{F}}=24.6 \mathrm{~Hz},{ }^{4} \mathrm{~J}_{\mathrm{H}-\mathrm{F}}=4.9 \mathrm{~Hz}\right) .{ }^{13} \mathrm{C}-\mathrm{NMR}\left(75.5 \mathrm{MHz} \mathrm{CDCl}_{3}\right) \delta 192.9(\mathrm{~d}$, $\left.{ }^{2} \mathrm{~J}_{\mathrm{C}-\mathrm{F}}=39 \mathrm{~Hz}, \mathrm{C}_{13}\right), 153.3\left(\mathrm{~d},{ }^{1} \mathrm{~J}_{\mathrm{C}-\mathrm{F}}=258 \mathrm{~Hz}, \mathrm{C}_{1}\right), 133.6\left(\mathrm{C}_{\mathrm{ar}}^{\mathrm{q}}\right), 133.1\left(\mathrm{C}_{\mathrm{ar}}^{\mathrm{q}}\right), 130.3(\mathrm{~d}$, $\left.\mathrm{J}=4 \mathrm{~Hz}, \mathrm{C}_{\mathrm{ar}}{ }^{\mathrm{H}}\right), 128.6\left(\mathrm{C}_{\mathrm{ar}}{ }^{\mathrm{H}}\right), 128.4\left(\mathrm{C}_{\mathrm{ar}}{ }^{\mathrm{q}}\right), 127.9\left(\mathrm{C}_{\mathrm{ar}}{ }^{\mathrm{H}}\right), 127.7\left(\mathrm{C}_{\mathrm{ar}}{ }^{\mathrm{H}}\right), 127.3(\mathrm{~d}, \mathrm{~J}=$ $\left.2 \mathrm{~Hz}, \mathrm{C}_{\mathrm{ar}}{ }^{\mathrm{H}}\right), 127.1\left(\mathrm{C}_{\mathrm{ar}}{ }^{\mathrm{H}}\right), 126.5\left(\mathrm{C}_{\mathrm{ar}}{ }^{\mathrm{H}}\right), 120.4\left(\mathrm{~d},{ }^{2} \mathrm{~J}_{\mathrm{C}-\mathrm{F}}=28 \mathrm{~Hz}, \mathrm{C}_{2}\right), 28.4\left(\mathrm{C}_{14}\right) . \mathrm{MS}(\mathrm{El})$ $214\left(\mathrm{M}^{+}\right), 179,171\left(\mathrm{M}^{+}-\mathrm{CH}_{3} \mathrm{CO}\right), 43\left(\mathrm{CH}_{3} \mathrm{CO}^{+}\right)$. IR (KBr) 3055, 2973, 2879, 1693, $1614,1392,1361,1190,1183,1093,928,815,790,740,529,470$. Anal. Calcd for $\mathrm{C}_{14} \mathrm{H}_{11}$ FO: C, 78.49; H, 5.18. Found: C, 78.47; H, 5.22.
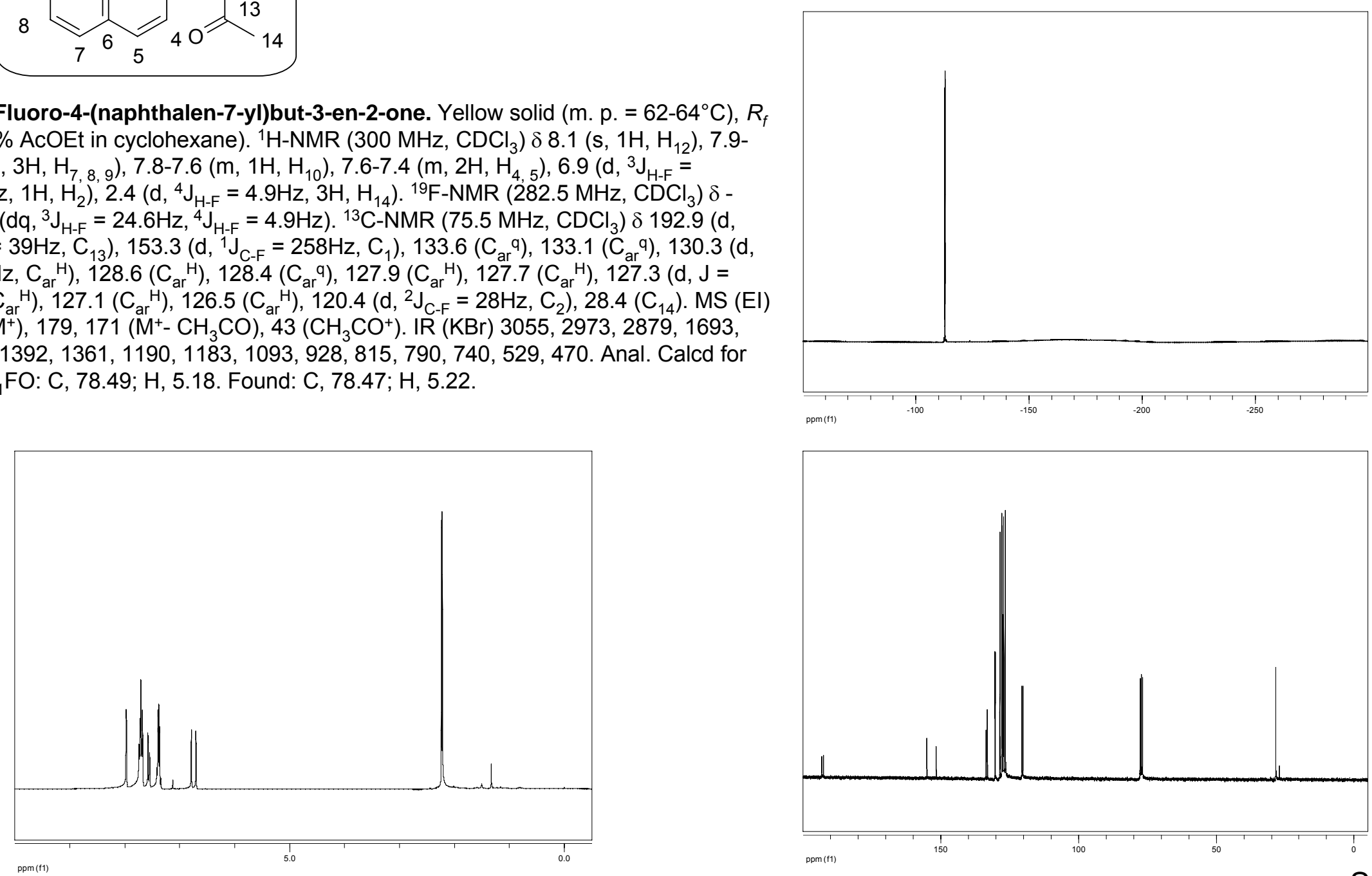


\section{Compound $\mathbf{4} \mathbf{a}_{\mathbf{3}}(\boldsymbol{E})$}

(E) 3-Fluoro-4-(4-cyanophenyl)but-3-en-2-one. Yellow powder (m. p. $\left.=85-87^{\circ} \mathrm{C}\right)$, $R_{f} 0.3$ (10\% AcOEt in cyclohexane). ${ }^{1} \mathrm{H}-\mathrm{NMR}\left(300 \mathrm{MHz}, \mathrm{CDCl}_{3}\right) \delta 7.7$ (dd, J = 8.7 Hz, $\left.4 \mathrm{H}, \mathrm{H}_{4,5}\right), 6.6\left(\mathrm{~d},{ }^{3} \mathrm{~J}_{\mathrm{H}-\mathrm{F}}=23.3 \mathrm{~Hz}, 1 \mathrm{H}, \mathrm{H}_{2}\right), 2.3\left(\mathrm{~d},{ }^{4} \mathrm{~J}_{\mathrm{H}-\mathrm{F}}=5.1 \mathrm{~Hz}, 3 \mathrm{H}, \mathrm{H}_{9}\right) .{ }^{19} \mathrm{~F}-\mathrm{NMR}$ $\left(282.5 \mathrm{MHz}, \mathrm{CDCl}_{3}\right) \delta-109.6\left(\mathrm{dq},{ }^{3} \mathrm{~J}_{\mathrm{H}-\mathrm{F}}=23.3 \mathrm{~Hz},{ }^{4} \mathrm{~J}_{\mathrm{H}-\mathrm{F}}=5.1 \mathrm{~Hz}\right) .{ }^{13} \mathrm{C}-\mathrm{NMR}(75.5$ $\left.\mathrm{MHz} \mathrm{CDCl}_{3}\right) \delta 193.2\left(\mathrm{~d}, 2 \mathrm{~J}_{\mathrm{C}-\mathrm{F}}=40 \mathrm{~Hz}, \mathrm{C}_{8}\right), 154.3\left(\mathrm{~d}, 1_{\mathrm{C}-\mathrm{F}}=265 \mathrm{~Hz}, \mathrm{C}_{1}\right), 136.0(\mathrm{~d}$, $\left.{ }^{3} \mathrm{~J}_{\mathrm{C}-\mathrm{F}}=11 \mathrm{~Hz}, \mathrm{C}_{3}\right), 132.2\left(\mathrm{C}_{5}\right), 130.9\left(\mathrm{~d}, \mathrm{~J}=3 \mathrm{~Hz}, \mathrm{C}_{4}\right), 118.9\left(\mathrm{C}_{7}\right), 118.0\left(\mathrm{~d},{ }^{2} \mathrm{~J}_{\mathrm{C}-\mathrm{F}}=\right.$ $\left.29 \mathrm{~Hz}, \mathrm{C}_{2}\right), 112.8\left(\mathrm{C}_{6}\right), 28.4\left(\mathrm{C}_{9}\right) . \mathrm{MS}(\mathrm{EI}) 189\left(\mathrm{M}^{+}\right), 174\left(\mathrm{M}^{+}-\mathrm{CH}_{3}\right), 146\left(\mathrm{M}^{+}-\mathrm{CH}_{3} \mathrm{CO}\right)$, $43\left(\mathrm{CH}_{3} \mathrm{CO}^{+}\right)$. IR (KBr) 3110, 3012, 2960, 2232, 1698, 1616, 1505, 1422, 1368,

1191, 1087, 912, 840, 700, 556, 516. Anal. Calcd for $\mathrm{C}_{11} \mathrm{H}_{8}$ FNO: C, 69.84; $\mathrm{H}, 4.26$; N, 7.40. Found: C, 69.85; H, 4.21; N, 7.33.
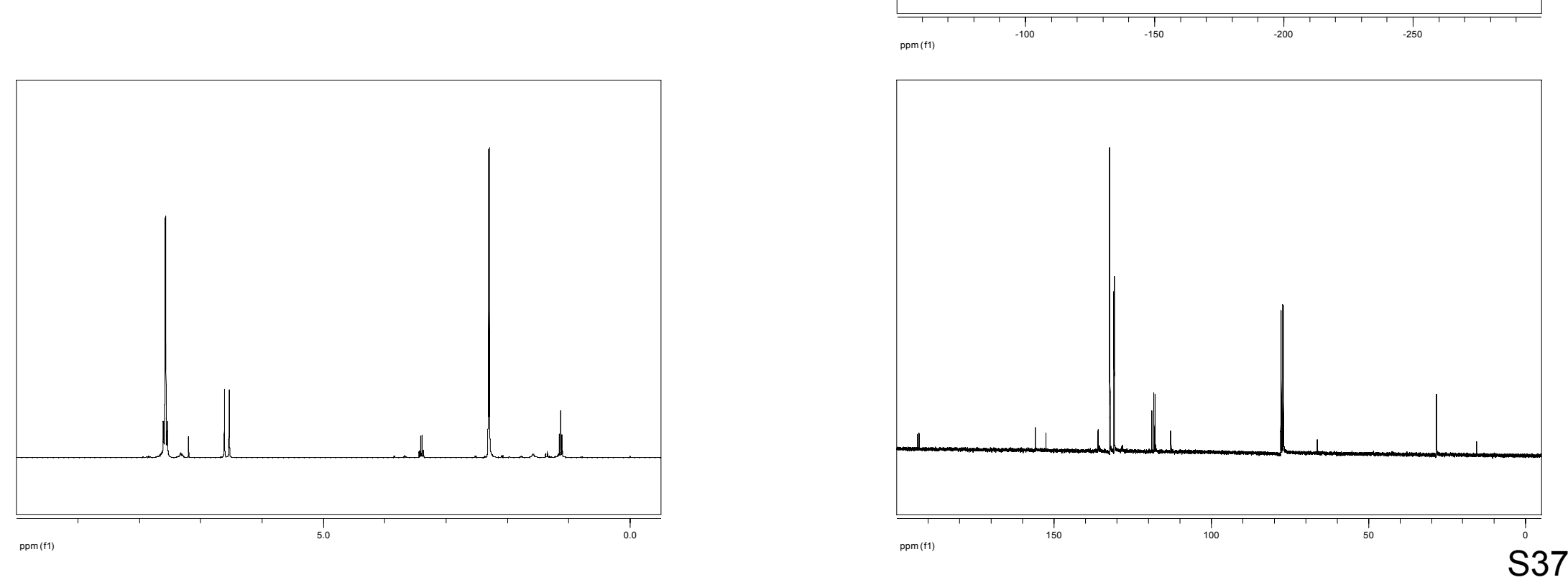


\section{Compound $\mathbf{4} \mathbf{a}_{4}(\boldsymbol{E})$}

(E) 3-Fluoro-4-(4-ethylbenzoate)but-3-en-2-one. Yellow powder (m. p. = 97$\left.99^{\circ} \mathrm{C}\right), R_{f} 0.2\left(5 \% \mathrm{AcOEt}\right.$ in cyclohexane). ${ }^{1} \mathrm{H}-\mathrm{NMR}\left(300 \mathrm{MHz}, \mathrm{CDCl}_{3}\right) \delta 8.1(\mathrm{~d}, \mathrm{~J}=$ $\left.8.2 \mathrm{~Hz}, 2 \mathrm{H}, \mathrm{H}_{5}\right), 7.7\left(\mathrm{~d}, \mathrm{~J}=8.3 \mathrm{~Hz}, 2 \mathrm{H}, \mathrm{H}_{4}\right), 6.7\left(\mathrm{~d},{ }^{3} \mathrm{~J}_{\mathrm{H}-\mathrm{F}}=23.7 \mathrm{~Hz}, 1 \mathrm{H}, \mathrm{H}_{2}\right), 3.9(\mathrm{~s}$ $\left.3 \mathrm{H}, \mathrm{H}_{8}\right), 2.3\left(\mathrm{~d},{ }^{4} \mathrm{~J}_{\mathrm{H}-\mathrm{F}}=4.9 \mathrm{~Hz}, 3 \mathrm{H}, \mathrm{H}_{10}\right) \cdot{ }^{19} \mathrm{~F}-\mathrm{NMR}\left(282.5 \mathrm{MHz}, \mathrm{CDCl}_{3}\right) \delta-111.0(\mathrm{dq}$, $\left.{ }^{3} \mathrm{~J}_{\mathrm{H}-\mathrm{F}}=23.7 \mathrm{~Hz},{ }^{4} \mathrm{~J}_{\mathrm{H}-\mathrm{F}}=5.4 \mathrm{~Hz}\right) .{ }^{13} \mathrm{C}-\mathrm{NMR}\left(75.5 \mathrm{MHz}, \mathrm{CDCl}_{3}\right) \delta 192.9\left(\mathrm{~d},{ }^{2} \mathrm{~J}_{\mathrm{C}-\mathrm{F}}=\right.$ $\left.39 \mathrm{~Hz}, \mathrm{C}_{9}\right), 166.7\left(\mathrm{C}_{7}\right), 153.7\left(\mathrm{~d},{ }^{1} \mathrm{~J}_{\mathrm{C}-\mathrm{F}}=262 \mathrm{~Hz}, \mathrm{C}_{1}\right), 135.6\left(\mathrm{~d}, \mathrm{~J}=11 \mathrm{~Hz}, \mathrm{C}_{6}\right), 130.6$ $\left(\mathrm{d},{ }^{3} \mathrm{~J}_{\mathrm{C}-\mathrm{F}}=5 \mathrm{~Hz}, \mathrm{C}_{3}\right), 130.0\left(\mathrm{~d}, \mathrm{~J}=3 \mathrm{~Hz}, \mathrm{C}_{5}\right), 129.5\left(\mathrm{C}_{4}\right), 118.8\left(\mathrm{~d},{ }^{2} \mathrm{~J}_{\mathrm{C}-\mathrm{F}}=28 \mathrm{~Hz}, \mathrm{C}_{2}\right)$, $52.4\left(\mathrm{C}_{8}\right), 28.3\left(\mathrm{C}_{10}\right)$. MS (EI) $222\left(\mathrm{M}^{+}\right), 207\left(\mathrm{M}^{+}-\mathrm{CH}_{3}\right), 191\left(\mathrm{M}^{+}-\mathrm{CH}_{3} \mathrm{O}\right), 163\left(\mathrm{M}^{+}-\right.$ $\left.\mathrm{CO}_{2} \mathrm{CH}_{3}\right), 43\left(\mathrm{CH}_{3} \mathrm{CO}^{+}\right)$. IR (KBr) 3066, 2960, 1721, 1637, 1433, 1364, 1320, 1280, $1200,1109,1057,1019,968,905,834,770,698,572$. Anal. Calcd for $\mathrm{C}_{12} \mathrm{H}_{11} \mathrm{FO}_{3}$ : C, 64.86; H, 4.99. Found: C, 64.84; H, 4.96.



\section{Compound $\mathbf{4} \mathbf{a}_{5}(\boldsymbol{E})$}

(E) 3-Fluoro-4-(4-nitrophenyl)but-3-en-2-one. Yellow solid (m. p. $\left.=89-91^{\circ} \mathrm{C}\right), R_{f}$ $0.2\left(5 \%\right.$ AcOEt in cyclohexane). ${ }^{1} \mathrm{H}-\mathrm{NMR}\left(300 \mathrm{MHz}^{\mathrm{C}} \mathrm{CDCl}_{3}\right) \delta 8.2(\mathrm{~d}, \mathrm{~J}=8.8 \mathrm{~Hz}, 2 \mathrm{H}$, $\left.\mathrm{H}_{5}\right), 7.7\left(\mathrm{~d}, \mathrm{~J}=8.6 \mathrm{~Hz}, 2 \mathrm{H}, \mathrm{H}_{4}\right), 6.7\left(\mathrm{~d},{ }^{3} \mathrm{~J}_{\mathrm{H}-\mathrm{F}}=22.9 \mathrm{~Hz}, 1 \mathrm{H}, \mathrm{H}_{2}\right), 2.4\left(\mathrm{~d},{ }^{4} \mathrm{~J}_{\mathrm{H}-\mathrm{F}}=5.2 \mathrm{~Hz}\right.$, $\left.3 \mathrm{H}, \mathrm{H}_{8}\right) .{ }^{19} \mathrm{~F}-\mathrm{NMR}\left(282.5 \mathrm{MHz}, \mathrm{CDCl}_{3}\right) \delta-119.0\left(\mathrm{dq},{ }^{3} \mathrm{~J}_{\mathrm{H}-\mathrm{F}}=22.6 \mathrm{~Hz},{ }^{4} \mathrm{~J}_{\mathrm{H}-\mathrm{F}}=5.4 \mathrm{~Hz}\right)$. ${ }^{13} \mathrm{C}-N M R\left(75.5 \mathrm{MHz}, \mathrm{CDCl}_{3}\right) \delta 192.9\left(\mathrm{~d},{ }^{2} \mathrm{~J}_{\mathrm{C}-\mathrm{F}}=40 \mathrm{~Hz}, \mathrm{C}_{7}\right), 154.1\left(\mathrm{~d},{ }^{1} \mathrm{~J}_{\mathrm{C}-\mathrm{F}}=266 \mathrm{~Hz}\right.$, $\left.\mathrm{C}_{1}\right), 147.8\left(\mathrm{C}_{6}\right), 137.6\left(\mathrm{~d}, \mathrm{~J}=11 \mathrm{~Hz}, \mathrm{C}_{3}\right), 130.9\left(\mathrm{~d}, \mathrm{~J}=3 \mathrm{~Hz}, \mathrm{C}_{5}\right), 123.4\left(\mathrm{C}_{4}\right), 117.2$ $\left(\mathrm{d},{ }^{2} \mathrm{~J}_{\mathrm{C}-\mathrm{F}}=29 \mathrm{~Hz}, \mathrm{C}_{2}\right), 28.1\left(\mathrm{C}_{8}\right) . \mathrm{MS}(\mathrm{El}) 209\left(\mathrm{M}^{+}\right), 192,162\left(\mathrm{M}^{+}-\mathrm{HNO}_{2}\right), 43$ $\left(\mathrm{CH}_{3} \mathrm{CO}^{+}\right)$. IR $(\mathrm{KBr}) 3011,1706,1595,1522,1342,1199,1086,904,846,751,689$ 532, 512. Anal. Calcd for $\mathrm{C}_{10} \mathrm{H}_{8} \mathrm{FNO}_{3}$ : C, 57.42; $\mathrm{H}, 3.85 ; \mathrm{N}, 6.70$. Found: $\mathrm{C}, 57.43$; $\mathrm{H}, 3.92 ; \mathrm{N}, 6.63$
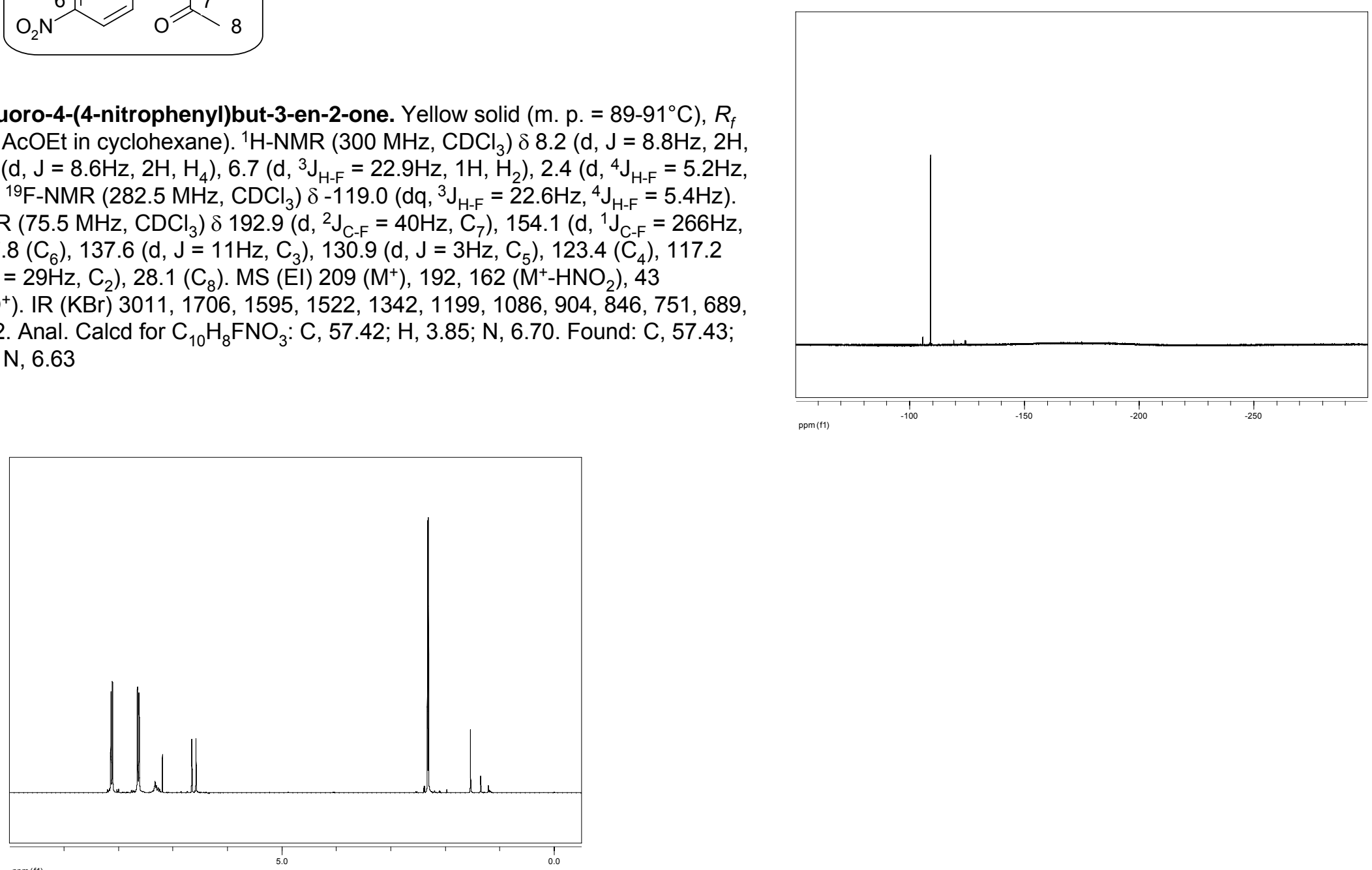


\section{Compound $\mathbf{4} \mathbf{a}_{7}(E)$}

(E) 3-Fluoro-6-phenylhex-3-en-2-one. Yellow oil, $R_{f} 0.2(5 \%$ AcOEt in cyclohexane). ${ }^{1} \mathrm{H}-\mathrm{NMR}\left(300 \mathrm{MHz}, \mathrm{CDCl}_{3}\right) \delta 7.3-7.0\left(\mathrm{~m}, 5 \mathrm{H}, \mathrm{H}_{6,7.8}\right), 5.7\left(\mathrm{dt},{ }^{3} \mathrm{~J}_{\mathrm{H}-\mathrm{F}}=\right.$ 22.0Hz, J = 8.0Hz, $\left.1 \mathrm{H}, \mathrm{H}_{2}\right), 2.9-2.7\left(\mathrm{~m}, 2 \mathrm{H}, \mathrm{H}_{4}\right), 2.7-2.5\left(\mathrm{~m}, 2 \mathrm{H}, \mathrm{H}_{3}\right), 2.2\left(\mathrm{~d},{ }^{4} \mathrm{~J}=\right.$ $\left.4.9 \mathrm{~Hz}, 3 \mathrm{H}, \mathrm{H}_{10}\right) \cdot{ }^{19} \mathrm{~F}-\mathrm{NMR}\left(282.5 \mathrm{MHz}, \mathrm{CDCl}_{3}\right) \delta-118.8\left(\mathrm{dq},{ }^{3} \mathrm{~J}_{\mathrm{H}-\mathrm{F}}=22.0 \mathrm{~Hz},{ }^{4} \mathrm{~J}=\right.$ $4.9 \mathrm{~Hz}) \cdot{ }^{13} \mathrm{C}-\mathrm{NMR}\left(75.5 \mathrm{MHz}, \mathrm{CDCl}_{3}\right) \delta 193.3\left(\mathrm{~d},{ }^{2} \mathrm{~J}_{\mathrm{C}-\mathrm{F}}=40 \mathrm{~Hz}, \mathrm{C}_{9}\right), 152.4\left(\mathrm{~d},{ }^{1} \mathrm{~J}_{\mathrm{C}-\mathrm{F}}=\right.$ $\left.256 \mathrm{~Hz}, \mathrm{C}_{1}\right), 139.6\left(\mathrm{C}_{5}\right), 127.3\left(\mathrm{C}_{6 / 7}\right), 127.2\left(\mathrm{C}_{6 / 7}\right), 125.0\left(\mathrm{C}_{8}\right), 119.2\left(\mathrm{~d},{ }^{2} \mathrm{~J}_{\mathrm{C}-\mathrm{F}}=18 \mathrm{~Hz}\right.$, $\left.\mathrm{C}_{2}\right), 34.0\left(\mathrm{~d},{ }^{4} \mathrm{~J}_{\mathrm{CF}}=2 \mathrm{~Hz}, \mathrm{C}_{4}\right), 25.8\left(\mathrm{C}_{10}\right), 25.7\left(\mathrm{C}_{3}\right) . \mathrm{MS}(\mathrm{El}) 192\left(\mathrm{M}^{+}\right), 172,91$ $\left(\mathrm{PhCH}_{2}{ }^{+}\right), 43\left(\mathrm{CH}_{3} \mathrm{CO}^{+}\right)$. IR (neat) 3063, 3027, 2927, 2860, 1708, 1640, 1365, 1105, 749, 700. Anal. Calcd for $\mathrm{C}_{12} \mathrm{H}_{13}$ FO: C, 74.98; $\mathrm{H}, 6.82$. Found: $\mathrm{C}, 75.49 ; \mathrm{H}, 7.17$.



\section{Compound $\mathbf{4} \mathbf{a}_{\mathbf{8}}(\boldsymbol{E})$}

(E) 3-Fluoro-6-(oxytert-butyldiphenylsilyl)hex-3-en-2-one. Pale yellow oil, $R_{f} 0.4$ (2\% AcOEt in cyclohexane). ${ }^{1} \mathrm{H}-\mathrm{NMR}\left(300 \mathrm{MHz} \mathrm{CDCl}_{3}\right) \delta 7.7-7.5\left(\mathrm{~m}, 4 \mathrm{H}, \mathrm{H}_{6}\right), 7.4-$ $7.2\left(\mathrm{~m}, 6 \mathrm{H}, \mathrm{H}_{7,8}\right), 5.8\left(\mathrm{dt},{ }^{3} \mathrm{~J}_{\mathrm{H}-\mathrm{F}}=21.8 \mathrm{~Hz},{ }^{3} \mathrm{~J}_{\mathrm{H}-\mathrm{H}}=7.9 \mathrm{~Hz}, 1 \mathrm{H}, \mathrm{H}_{2}\right), 3.7\left(\mathrm{dt},{ }^{5} \mathrm{~J}_{\mathrm{H}-\mathrm{F}}=\right.$ $\left.1.0 \mathrm{~Hz},{ }^{3} \mathrm{~J}_{\mathrm{H}-\mathrm{H}}=6.4 \mathrm{~Hz}, 2 \mathrm{H}, \mathrm{H}_{4}\right), 2.8-2.6\left(\mathrm{~m}, 2 \mathrm{H}, \mathrm{H}_{3}\right), 2.2\left(\mathrm{~d},{ }^{4} \mathrm{~J}_{\mathrm{H}-\mathrm{F}}=4.9 \mathrm{~Hz}, 3 \mathrm{H}, \mathrm{H}_{12}\right)$, $0.9\left(\mathrm{~s}, 9 \mathrm{H}, \mathrm{H}_{10}\right) .{ }^{19} \mathrm{~F}-\mathrm{NMR}\left(282.5 \mathrm{MHz}, \mathrm{CDCl}_{3}\right) \delta-118.1(\mathrm{~m}) .{ }^{13} \mathrm{C}-\mathrm{NMR}(75.5 \mathrm{MHz}$, $\left.\mathrm{CDCl}_{3}\right) \delta 194.9\left(\mathrm{~d},{ }^{2} \mathrm{~J}_{\mathrm{C}-\mathrm{F}}=40 \mathrm{~Hz}, \mathrm{C}_{11}\right), 154.3\left(\mathrm{~d},{ }^{1} \mathrm{~J}_{\mathrm{C}-\mathrm{F}}=255 \mathrm{~Hz}, \mathrm{C}_{1}\right), 136.0\left(\mathrm{C}_{7}\right)$, $134.0\left(\mathrm{C}_{5}\right), 130.1\left(\mathrm{C}_{8}\right), 128.1\left(\mathrm{C}_{6}\right), 118.9\left(\mathrm{~d},{ }^{2} \mathrm{~J}_{\mathrm{C}-\mathrm{F}}=20 \mathrm{~Hz}, \mathrm{C}_{2}\right), 63.2\left(\mathrm{~d}, 4 \mathrm{~J}_{\mathrm{C}-\mathrm{F}}=2 \mathrm{~Hz}\right.$, $\left.\mathrm{C}_{4}\right), 29.2\left(\mathrm{~d},{ }^{3} \mathrm{~J}_{\mathrm{C}-\mathrm{F}}=5 \mathrm{~Hz}, \mathrm{C}_{3}\right), 28.1\left(\mathrm{~d},{ }^{3} \mathrm{~J}_{\mathrm{C}-\mathrm{F}}=3 \mathrm{~Hz}, \mathrm{C}_{12}\right), 27.3\left(\mathrm{C}_{10}\right), 19.6\left(\mathrm{C}_{9}\right) . \mathrm{MS}$ (EI) $313\left(\mathrm{M}^{+}\right.$-tBu), 201, 199, 181, 141, $43\left(\mathrm{CH}_{3} \mathrm{CO}^{+}\right)$. IR (neat) 3394, 3072, 2959 2858, 1709, 1643, 1472, 1427, 1365, 1194, 1112, 938, 823, 704, 687, 614, 505. Anal. Calcd for $\mathrm{C}_{22} \mathrm{H}_{27} \mathrm{FO}_{2} \mathrm{Si}$ : C, 71.31; $\mathrm{H}, 7.34$. Found: $\mathrm{C}, 71.35 ; \mathrm{H}, 7.39$.
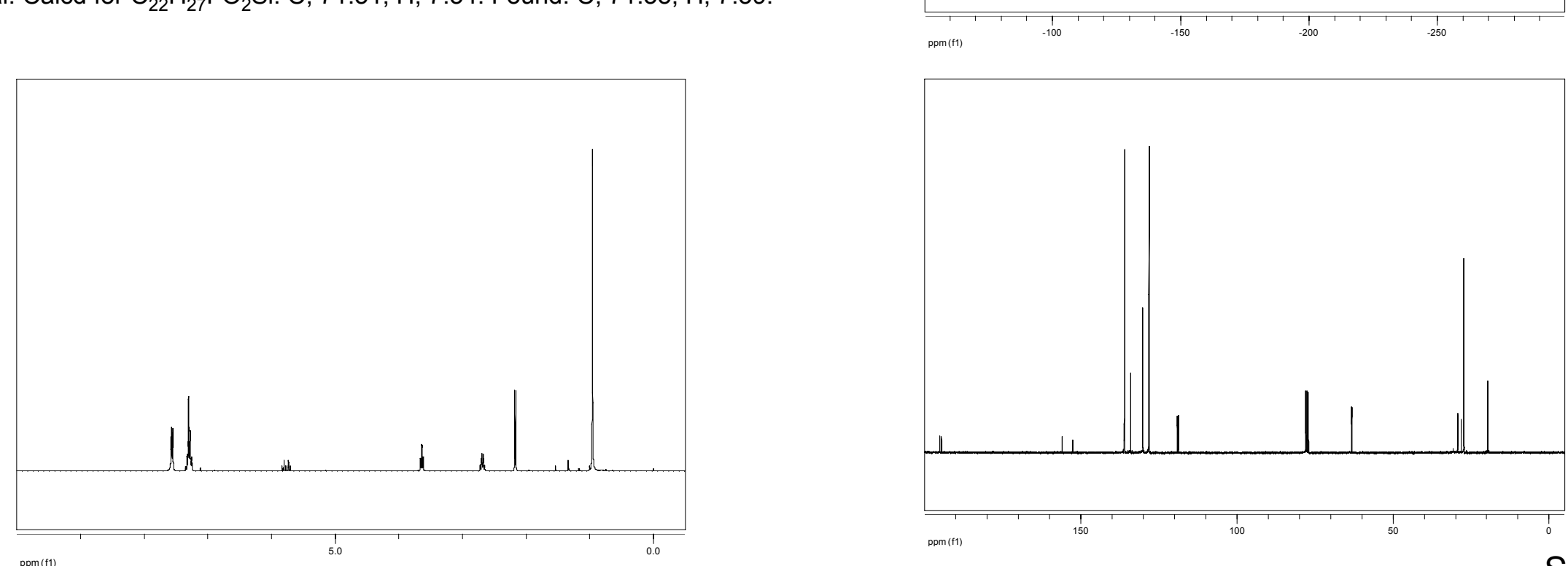
Procedure for the obtention of $(E)$ fluorinated benzylketones $4 b(E)$ from bromofluoroolefins $2(Z)$. To a mixture of potassium tert-butoxide (4 eq.), freshly synthesized 1-(2-Ethoxyvinyl)benzene (4 eq.) in anhydrous THF (5 $\mathrm{mL} / \mathrm{mmol}$ of $t$-BuOK) at $-78^{\circ} \mathrm{C}$ under argon was added dropwise $n$-BuLi in hexanes (1.6M, 2 eq.). The mixture was stirred for $30 \mathrm{~min}$ at $-78^{\circ} \mathrm{C}$ and then a solution of dry zincchloride (8 eq.) in THF ( $4 \mathrm{~mL} / \mathrm{mmol}$ of $\mathrm{ZnCl}_{2}$ ) was added dropwise. After $10 \mathrm{~min}$, the cooling bath was removed and the solution was allowed to warm to room temperature for $30 \mathrm{~min}$. The mixture was then added slowly to a solution of palladiumdiacetate ( 0.05 eq.), triphenylphosphine ( 0.1 eq.) and $(Z)$ bromofluoroolefin $2(Z)$ (1 eq.) in anhydrous THF $\left(10 \mathrm{~mL} / \mathrm{mmol}\right.$ of bromofluoroolefin) at $70^{\circ} \mathrm{C}$ under argon. The mixture was stirred for $1 \mathrm{~h}$. After the coupling reaction was completed, controlled by monitoring ${ }^{19} \mathrm{~F}-\mathrm{NMR}$ signal of the reaction mixture, the solution was allowed to cool to room temperature. $\mathrm{HCl}$ aq. (1N) was added and the mixture was stirred for $15 \mathrm{~min}$. The mixture was then extracted with $\mathrm{Et}_{2} \mathrm{O}$ (x 3), washed with brine and the combined organic layers were dried over $\mathrm{MgSO}_{4}$. After filtration and concentration under reduced pressure, the residue was purified by chromatography on silica gel (eluent: 2\% AcOEt in cyclohexane), affording (E) benzylketone $\mathbf{4 b}(\boldsymbol{E})$. 


\section{Compound $\mathbf{4} \mathbf{b}_{2}(\boldsymbol{E})$}

(E) 3-Fluoro-1,6-diphenylhex-3-en-2-one. Yellow oil, $R_{f} 0.5$ (5\% AcOEt in cyclohexane). ${ }^{1} \mathrm{H}-\mathrm{NMR}\left(300 \mathrm{MHz}, \mathrm{CDCl}_{3}\right) \delta 7.3-7.1\left(\mathrm{~m}, 10 \mathrm{H}, \mathrm{H}_{6,7,8,12,13,14}\right), 5.7$ $\left(\mathrm{dt},{ }^{3} \mathrm{~J}_{\mathrm{H}-\mathrm{F}}=22.2 \mathrm{~Hz},{ }^{3} \mathrm{~J}_{\mathrm{H}-\mathrm{H}}=7.9 \mathrm{~Hz}, 1 \mathrm{H}, \mathrm{H}_{2}\right), 3.8\left(\mathrm{~d},{ }^{4} \mathrm{~J}_{\mathrm{H}-\mathrm{F}}=3.8 \mathrm{~Hz}, 2 \mathrm{H}, \mathrm{H}_{10}\right), 2.8-2.7$ $\left(\mathrm{m}, 2 \mathrm{H}, \mathrm{H}_{4}\right), 2.6-2.5\left(\mathrm{~m}, 2 \mathrm{H}, \mathrm{H}_{3}\right)$. ${ }^{19} \mathrm{~F}-\mathrm{NMR}\left(282.5 \mathrm{MHz}, \mathrm{CDCl}_{3}\right) \delta-120.3\left(\mathrm{dt},{ }^{3} \mathrm{~J}_{\mathrm{H}-\mathrm{F}}=\right.$ $\left.23.0 \mathrm{~Hz},{ }^{4} \mathrm{~J}_{\mathrm{H}-\mathrm{F}}=2.3 \mathrm{~Hz}\right) .{ }^{13} \mathrm{C}-\mathrm{NMR}\left(75.5 \mathrm{MHz}, \mathrm{CDCl}_{3}\right) \delta 194.6\left(\mathrm{~d},{ }^{2} \mathrm{~J}_{\mathrm{C}-\mathrm{F}}=39 \mathrm{~Hz}, \mathrm{C}_{9}\right)$, $154.0\left(\mathrm{~d},{ }^{1} \mathrm{~J}_{\mathrm{C}-\mathrm{F}}=256 \mathrm{~Hz}, \mathrm{C}_{1}\right), 141.4\left(\mathrm{C}_{5}\right), 133.7\left(\mathrm{C}_{11}\right), 130.4\left(\mathrm{C}_{12 / 13}\right), 129.3\left(\mathrm{C}_{12 / 13}\right)$, $129.2\left(\mathrm{C}_{6 / 7}\right), 129.1\left(\mathrm{C}_{6 / 7}\right), 127.8\left(\mathrm{C}_{14}\right), 126.8\left(\mathrm{C}_{8}\right), 122.4\left(\mathrm{~d},{ }^{2} \mathrm{~J}_{\mathrm{C}-\mathrm{F}}=18 \mathrm{~Hz}, \mathrm{C}_{2}\right), 47.2$ $\left(\mathrm{d},{ }^{3} \mathrm{~J}_{\mathrm{C}-\mathrm{F}}=2 \mathrm{~Hz}, \mathrm{C}_{10}\right), 35.8\left(\mathrm{~d},{ }^{4} \mathrm{~J}_{\mathrm{C}-\mathrm{F}}=2 \mathrm{~Hz}, \mathrm{C}_{4}\right), 27.6\left(\mathrm{~d},{ }^{3} \mathrm{~J}_{\mathrm{C}-\mathrm{F}}=5 \mathrm{~Hz}, \mathrm{C}_{3}\right) \cdot \mathrm{MS}(\mathrm{El})$ $268\left(\mathrm{M}^{+}\right), 177\left(\mathrm{M}^{+}-\mathrm{PhCH}_{2}\right), 157,91\left(\mathrm{PhCH}_{2}^{+}\right)$. IR (neat) 3085, 3062, 3028, 2927, 2860, 1704, 1640, 1496, 1454, 1358, 1135, 1080, 749, 729, 698.Anal. Calcd for $\mathrm{C}_{18} \mathrm{H}_{17} \mathrm{FO}: \mathrm{C}, 81.57 ; \mathrm{H}, 6.39$. Found : C, 81.86; H, 6.51.
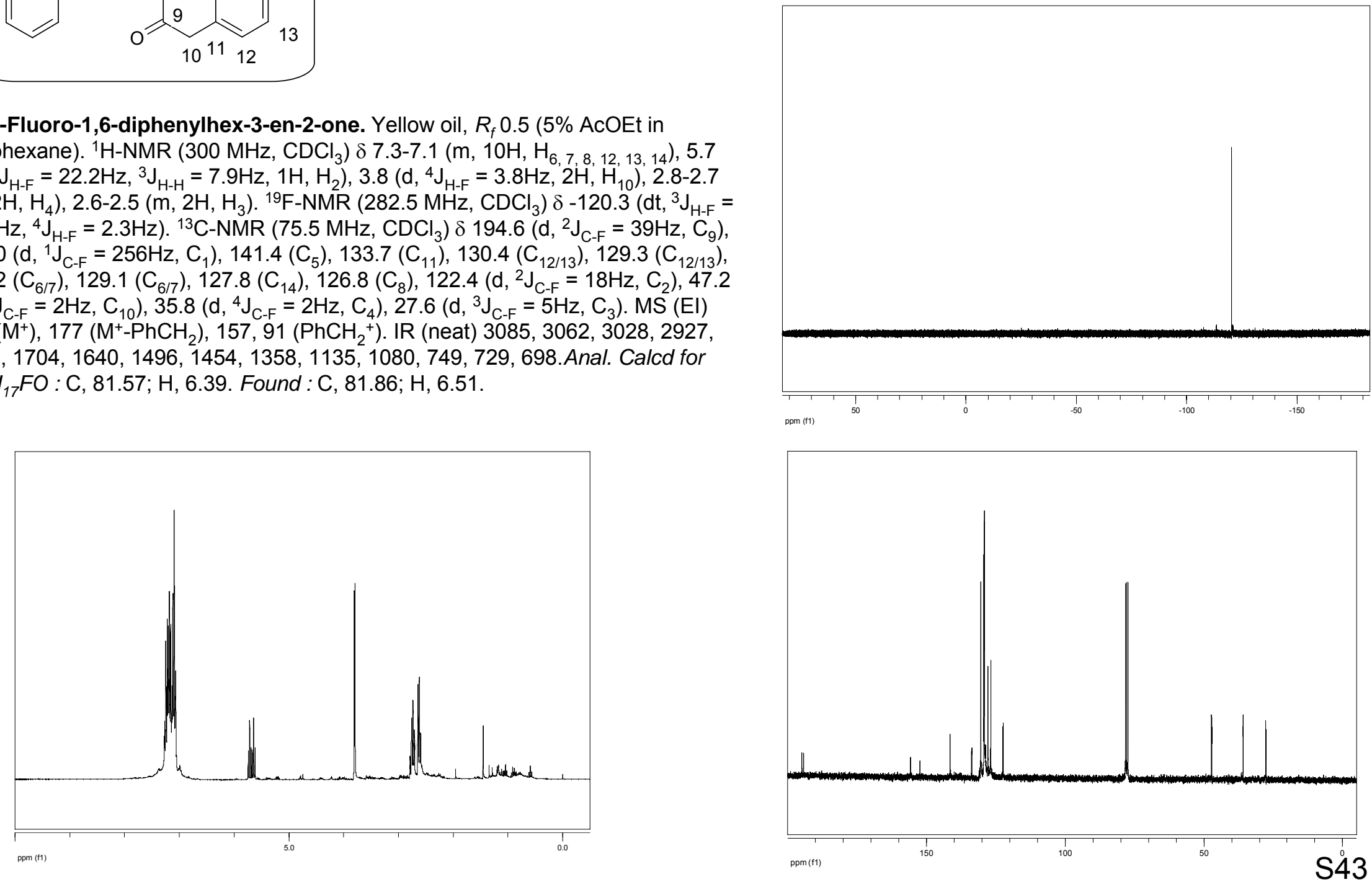
Procedure for the obtention of $(E)$ fluorinated propylketones $4 c(E)$ from bromofluoroolefins $2(Z)$. To a mixture of potassium tert-butoxide (4 eq.), butenylethylether (4 eq.) in anhydrous THF ( $5 \mathrm{~mL} / \mathrm{mmol}$ of $t$-BuOK) at $-78^{\circ} \mathrm{C}$ under argon was added dropwise $n$-BuLi in hexanes (1.6M, 4 eq.). The mixture was stirred for $30 \mathrm{~min}$ at $-78^{\circ} \mathrm{C}$ and then a solution of dry zincchloride (8 eq.) in THF ( $4 \mathrm{~mL} / \mathrm{mmol}$ of $\mathrm{ZnCl}_{2}$ ) was added dropwise. After $10 \mathrm{~min}$, the cooling bath was removed and the solution was allowed to warm to room temperature for $30 \mathrm{~min}$. The mixture was then added slowly to a solution of palladiumdiacetate (0.05 eq.), triphenylphosphine ( 0.1 eq.) and $(Z)$ bromofluoroolefin $2(Z)(1$ eq.) in anhydrous THF ( $10 \mathrm{~mL} / \mathrm{mmol}$ of bromofluoroolefin) at $55^{\circ} \mathrm{C}$ under argon. The mixture was stirred for $1 \mathrm{~h}$. After the reaction was completed, controlled by monitoring ${ }^{19} \mathrm{~F}-\mathrm{NMR}$ signal of the reaction mixture, the solution was allowed to cool to room temperature. $\mathrm{HCl}$ aq. $(1 \mathrm{~N})$ was added and the mixture was stirred for $15 \mathrm{~min}$. The mixture was then extracted with $\mathrm{Et}_{2} \mathrm{O}$ (x 3), washed with brine and the combined organic layers were dried over $\mathrm{MgSO}_{4}$. After filtration and concentration under reduced pressure, the residue was purified by chromatography on silica gel (eluent: cyclohexane/AcOEt), affording (E) propylketone $\mathbf{4 c}(\boldsymbol{E})$. 


\section{Compound $4 \mathbf{c}_{1}(E)$}

(E) 2-Fluoro-1-(4-methoxyphenyl)hex-1-en-3-one. Yellow oil, $R_{f} 0.4$ (5\% AcOEt in cyclohexane). ${ }^{1} \mathrm{H}-\mathrm{NMR}\left(300 \mathrm{MHz}, \mathrm{CDCl}_{3}\right) \delta 7.7\left(\mathrm{~d}, \mathrm{~J}=9.0 \mathrm{~Hz}, 2 \mathrm{H}, \mathrm{H}_{4}\right), 6.9(\mathrm{~d}, \mathrm{~J}=$ $\left.8.7 \mathrm{~Hz}, 2 \mathrm{H}, \mathrm{H}_{5}\right), 6.6\left(\mathrm{~d},{ }^{3} \mathrm{~J}_{\mathrm{H}-\mathrm{F}}=26.6 \mathrm{~Hz}, 1 \mathrm{H}, \mathrm{H}_{2}\right), 3.8\left(\mathrm{~s}, 3 \mathrm{H}, \mathrm{H}_{7}\right), 2.7\left(\mathrm{dt},{ }^{4} \mathrm{~J}_{\mathrm{H}-\mathrm{F}}=\right.$ $\left.3.6 \mathrm{~Hz},{ }^{3} \mathrm{~J}_{\mathrm{H}-\mathrm{H}}=7.2 \mathrm{~Hz}, 2 \mathrm{H}, \mathrm{H}_{9}\right), 1.7\left(\mathrm{~h},{ }^{3} \mathrm{~J}_{\mathrm{H}-\mathrm{H}}=7.4 \mathrm{~Hz}, 2 \mathrm{H}, \mathrm{H}_{10}\right), 1.0\left(\mathrm{t},{ }^{3} \mathrm{~J}_{\mathrm{H}-\mathrm{H}}=7.4 \mathrm{~Hz}\right.$ $\left.3 \mathrm{H}, \mathrm{H}_{11}\right) \cdot{ }^{19} \mathrm{~F}-\mathrm{NMR}\left(282.5 \mathrm{MHz}, \mathrm{CDCl}_{3}\right) \delta-117.7\left(\mathrm{dt},{ }^{3} \mathrm{~J}_{\mathrm{H}-\mathrm{F}}=25.8 \mathrm{~Hz},{ }^{4} \mathrm{~J}_{\mathrm{H}-\mathrm{F}}=3.8 \mathrm{~Hz},\right)$. ${ }^{13} \mathrm{C}-\mathrm{NMR}\left(75.5 \mathrm{MHz}, \mathrm{CDCl}_{3}\right) \delta 195.5\left(\mathrm{~d},{ }^{2} \mathrm{~J}_{\mathrm{C}-\mathrm{F}}=38 \mathrm{~Hz}, \mathrm{C}_{8}\right), 160.6\left(\mathrm{C}_{6}\right), 152.9(\mathrm{~d}$,

$\left.{ }^{1} \mathrm{~J}_{\mathrm{C}-\mathrm{F}}=254 \mathrm{~Hz}, \mathrm{C}_{1}\right), 132.3\left(\mathrm{C}_{4}\right), 123.3\left(\mathrm{~d},{ }^{3} \mathrm{~J}_{\mathrm{C}-\mathrm{F}}=10 \mathrm{~Hz}, \mathrm{C}_{3}\right), 120.4\left(\mathrm{~d},{ }^{2} \mathrm{~J}_{\mathrm{C}-\mathrm{F}}=29 \mathrm{~Hz}\right.$, $\left.\mathrm{C}_{2}\right), 113.7\left(\mathrm{C}_{5}\right), 55.4\left(\mathrm{C}_{7}\right), 42.1\left(\mathrm{C}_{9}\right), 16.8\left(\mathrm{C}_{10}\right), 13.8\left(\mathrm{C}_{11}\right) . \mathrm{MS}(\mathrm{El}) 222\left(\mathrm{M}^{+}\right), 193$ $\left(\mathrm{M}^{+}-\mathrm{CH}_{3} \mathrm{CH}_{2}\right), 179\left(\mathrm{M}^{+}-\mathrm{CH}_{3} \mathrm{CH}_{2} \mathrm{CH}_{2}\right), 136,108$. IR (neat) 3021, 2964, 2936, 2876, 2839, 1701, 1602, 1512, 1464, 1380, 1305, 1258, 1179, 1154, 1033, 875, 831, 598. Anal. Calcd for $\mathrm{C}_{13} \mathrm{H}_{15} \mathrm{FO}_{2}$ : C, 70.25; $\mathrm{H}, 6.80$. Found: $\mathrm{C}, 70.27 ; \mathrm{H}, 6.89$.
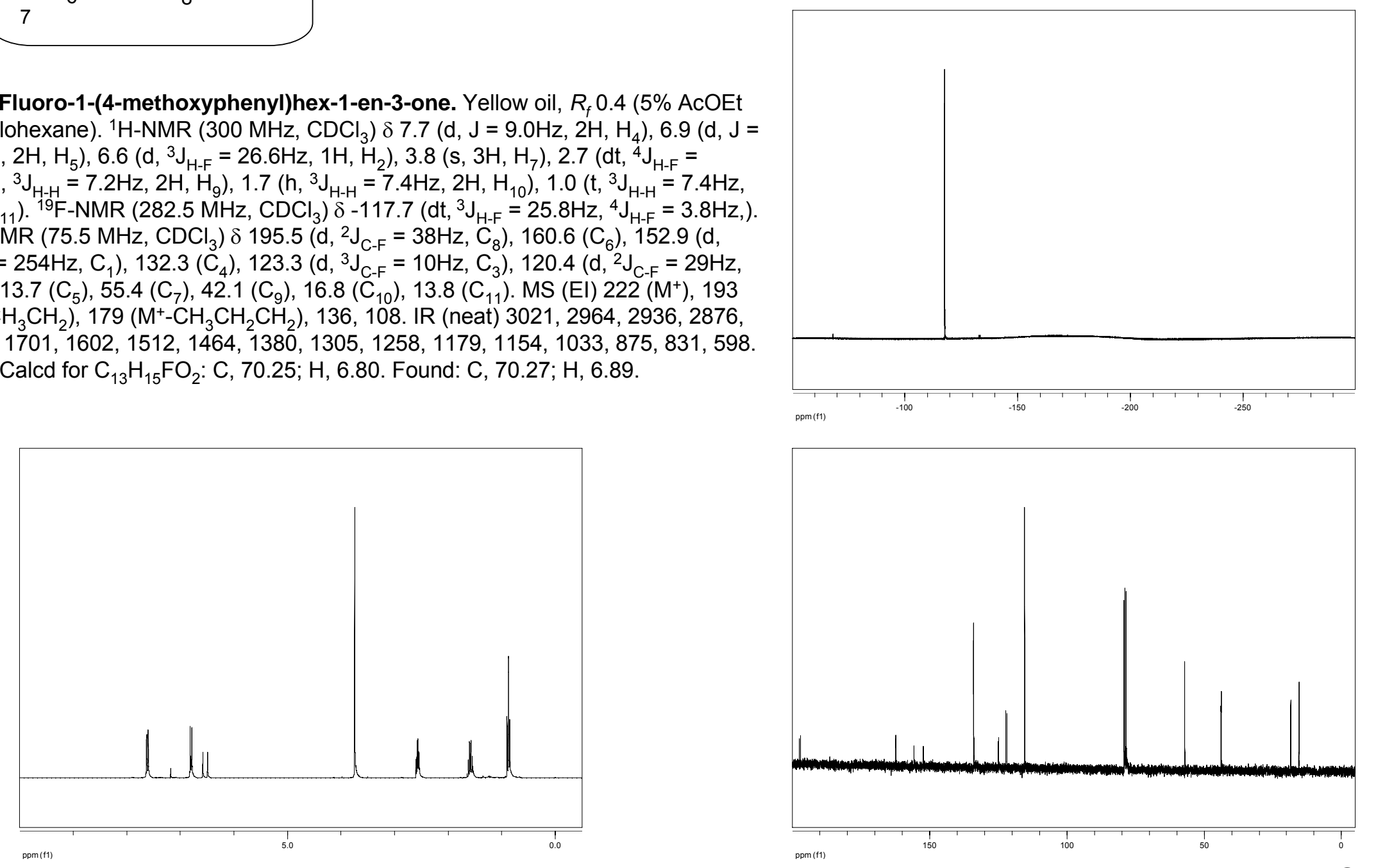


\section{Compound $\mathbf{4} \mathbf{c}_{2}(E)$}

(E) 4-Fluoro-1-phenyloct-3-en-5-one. Yellow oil, $R_{f} 0.6(5 \%$ AcOEt in cyclohexane). ${ }^{1} \mathrm{H}-\mathrm{NMR}\left(300 \mathrm{MHz} \mathrm{CDCl}_{3}\right) \delta 7.3-7.1\left(\mathrm{~m}, 5 \mathrm{H}, \mathrm{H}_{6,7,8}\right), 5.6\left(\mathrm{dt}^{3} \mathrm{~J}_{\mathrm{H}-\mathrm{F}}=\right.$ $\left.22.5 \mathrm{~Hz},{ }^{3} \mathrm{~J}_{\mathrm{H}-\mathrm{H}}=7.7 \mathrm{~Hz}, 1 \mathrm{H}, \mathrm{H}_{2}\right), 2.7\left(\mathrm{~m}, 2 \mathrm{H}, \mathrm{H}_{4}\right), 2.5\left(\mathrm{~m}, 2 \mathrm{H}, \mathrm{H}_{3}\right), 2.4\left(\mathrm{td},{ }^{3} \mathrm{~J}_{\mathrm{H}-\mathrm{H}}=\right.$

$\left.7.2 \mathrm{~Hz},{ }^{4} \mathrm{~J}_{\mathrm{H}-\mathrm{F}}=3.3 \mathrm{~Hz}, 2 \mathrm{H}, \mathrm{H}_{10}\right), 1.6\left(\mathrm{~m}, 2 \mathrm{H}, \mathrm{H}_{11}\right), 0.9\left(\mathrm{t},{ }^{3} \mathrm{~J}_{\mathrm{H}-\mathrm{H}}=7.4 \mathrm{~Hz}, 3 \mathrm{H}, \mathrm{H}_{12}\right) .{ }^{19} \mathrm{~F}-$ $\operatorname{NMR}\left(282.5 \mathrm{MHz}, \mathrm{CDCl}_{3}\right) \delta-121.2\left(\mathrm{~d},{ }^{3} \mathrm{~J}_{\mathrm{H}-\mathrm{F}}=22.6 \mathrm{~Hz}\right) .{ }^{13} \mathrm{C}-\mathrm{NMR}\left(75.5 \mathrm{MHz}, \mathrm{CDCl}_{3}\right)$ $\delta 197.1\left(\mathrm{~d},{ }^{2} \mathrm{~J}_{\mathrm{C}-\mathrm{F}}=38 \mathrm{~Hz}, \mathrm{C}_{9}\right), 153.8\left(\mathrm{~d},{ }^{1} \mathrm{~J}_{\mathrm{C}-\mathrm{F}}=256 \mathrm{~Hz}, \mathrm{C}_{1}\right), 140.9\left(\mathrm{C}_{5}\right), 128.6\left(\mathrm{C}_{6 / 7}\right)$, $128.5\left(\mathrm{C}_{6 / 7}\right), 126.2\left(\mathrm{C}_{8}\right), 120.2\left(\mathrm{~d},{ }^{2} \mathrm{~J}_{\mathrm{C}-\mathrm{F}}=18 \mathrm{~Hz}, \mathrm{C}_{2}\right), 41.8\left(\mathrm{C}_{10}\right), 35.4\left(\mathrm{C}_{4}\right), 26.9(\mathrm{~d}$, $\left.3 \mathrm{~J}_{\mathrm{C}-\mathrm{F}}=5 \mathrm{~Hz}, \mathrm{C}_{3}\right), 16.6\left(\mathrm{C}_{11}\right), 13.7\left(\mathrm{C}_{12}\right) . \mathrm{MS}(\mathrm{EI}) 220\left(\mathrm{M}^{+}\right), 157,128\left(\mathrm{M}^{+}-\mathrm{PhCH}_{3}\right)$, 104, $91\left(\mathrm{PhCH}_{2}^{+}\right)$. IR (neat) 3028, 2964, 2931, 2875, 1706, 1643, 1454, 1378, 1147, 1104, 748, 699. Anal. Calcd for $\mathrm{C}_{14} \mathrm{H}_{17}$ FO: C, 76.33; H, 7.78. Found: C, 76.45; $\mathrm{H}$, 7.96 .

Procedure for the obtention of $(E)$ fluorinated dihydrodioxine $7(E)$ from bromofluoroolefins $2(Z)$. To 1,4-diox2-ene (2 eq.) in anhydrous THF ( $5 \mathrm{~mL} / \mathrm{mmol}$ of enol ether) at $-30^{\circ} \mathrm{C}$ under argon was added dropwise $t$-BuLi in hexanes (1.6M, 2 eq.). The mixture was stirred for $1 \mathrm{~h}$ at $-20^{\circ} \mathrm{C}$ and then a solution of dry zincchloride (4 eq.) in THF $\left(4 \mathrm{~mL} / \mathrm{mmol}\right.$ of $\mathrm{ZnCl}_{2}$ ) was added dropwise at $-20^{\circ} \mathrm{C}$. After $10 \mathrm{~min}$, the cooling bath was removed and the solution was allowed to warm to room temperature for $30 \mathrm{~min}$. The mixture was then added slowly to a solution of palladiumdiacetate ( 0.05 eq.), triphenylphosphine ( 0.1 eq.) and $(Z)$ bromofluoroolefin $2(Z)$ (1 eq.) in anhydrous THF $\left(10 \mathrm{~mL} / \mathrm{mmol}\right.$ of bromofluoroolefin) at $70^{\circ} \mathrm{C}$ under argon. The mixture was stirred for $1 \mathrm{~h}$. After the coupling reaction was completed, controlled by monitoring ${ }^{19} \mathrm{~F}$-NMR signal of the reaction mixture, $\mathrm{NH}_{4} \mathrm{Cl}$ aq. (sat.) was added and the mixture was stirred for $5 \mathrm{~min}$. The mixture was then extracted with $\mathrm{Et}_{2} \mathrm{O}$ (x 3), washed with brine and the combined organic layers were dried over $\mathrm{MgSO}_{4}$. After filtration and concentration under reduced pressure, the residue was purified by chromatography on basic alumina (eluent: cyclohexane), affording (E) fluorinated dihydrodioxine $7(E)$. 


\section{Compound $7_{1}(E)$}

(E) 5-(1-Fluoro-2-(4-methoxyphenyl)vinyl)-2,3-dihydro-1,4-dioxene. Yellow oil, $R_{f} 0.4$ (cyclohexane, Aluminium oxide neutral). ${ }^{1} \mathrm{H}-\mathrm{NMR}\left(300 \mathrm{MHz}, \mathrm{CDCl}_{3}\right) \delta$ $7.1\left(\mathrm{~d}, \mathrm{~J}=9.1 \mathrm{~Hz}, 2 \mathrm{H}, \mathrm{H}_{4}\right), 6.7\left(\mathrm{~d}, \mathrm{~J}=8.7 \mathrm{~Hz}, 2 \mathrm{H}, \mathrm{H}_{5}\right), 6.4\left(\mathrm{~s}, 1 \mathrm{H}, \mathrm{H}_{9}\right), 6.1\left(\mathrm{~d},{ }^{3} \mathrm{~J}_{\mathrm{H}-\mathrm{F}}\right.$ $\left.=22.2 \mathrm{~Hz}, 1 \mathrm{H}, \mathrm{H}_{2}\right), 4.0-3.9\left(\mathrm{~m}, 4 \mathrm{H}, \mathrm{H}_{10,11}\right), 3.7\left(\mathrm{~s}, 3 \mathrm{H}, \mathrm{H}_{7}\right) .{ }^{19} \mathrm{~F}-\mathrm{NMR}(282.5 \mathrm{MHz}$, $\left.\mathrm{CDCl}_{3}\right) \delta-113.8\left(\mathrm{~d},{ }^{3} \mathrm{~J}_{\mathrm{H}-\mathrm{F}}=23.0 \mathrm{~Hz}\right) \cdot{ }^{13} \mathrm{C}-\mathrm{NMR}\left(75.5 \mathrm{MHz}, \mathrm{CDCl}_{3}\right) \delta 157.5\left(\mathrm{C}_{6}\right)$, $149.6\left(\mathrm{~d},{ }^{1} \mathrm{~J}_{\mathrm{C}-\mathrm{F}}=238 \mathrm{~Hz}, \mathrm{C}_{1}\right), 129.7\left(\mathrm{C}_{3}\right), 129.1\left(\mathrm{~d}, \mathrm{~J}=2 \mathrm{~Hz}, \mathrm{C}_{4}\right), 129.0\left(\mathrm{~d},{ }^{3} \mathrm{~J}_{\mathrm{C}-\mathrm{F}}=\right.$ $\left.6 \mathrm{~Hz}, \mathrm{C}_{9}\right), 124.4\left(\mathrm{~d},{ }^{2} \mathrm{~J}_{\mathrm{CF}}=12 \mathrm{~Hz}, \mathrm{C}_{8}\right), 112.3\left(\mathrm{C}_{5}\right), 108.3\left(\mathrm{~d},{ }^{2} \mathrm{~J}_{\mathrm{C}-\mathrm{F}}=30 \mathrm{~Hz}, \mathrm{C}_{2}\right)$ $63.6\left(\mathrm{C}_{10 / 11}\right), 62.8\left(\mathrm{C}_{10 / 11}\right), 54.1\left(\mathrm{C}_{7}\right)$. MS (El) $236\left(\mathrm{M}^{+}\right), 216,179\left(\mathrm{M}^{+}-\mathrm{CH}_{2}-\mathrm{O}-\right.$ $\left.\mathrm{CH}=\mathrm{CH}_{2}\right)$, 159. IR (neat) 3107, 2935, 2879, 1656, 1608, 1511, 1300, 1252,

1160, 1100, 1034, 922. Anal. Calcd for $\mathrm{C}_{13} \mathrm{H}_{13} \mathrm{FO}_{3}: \mathrm{C}, 66.09 ; \mathrm{H}, 5.55$. Found : $\mathrm{C}$, $64.94 ; \mathrm{H}, 5.52$.
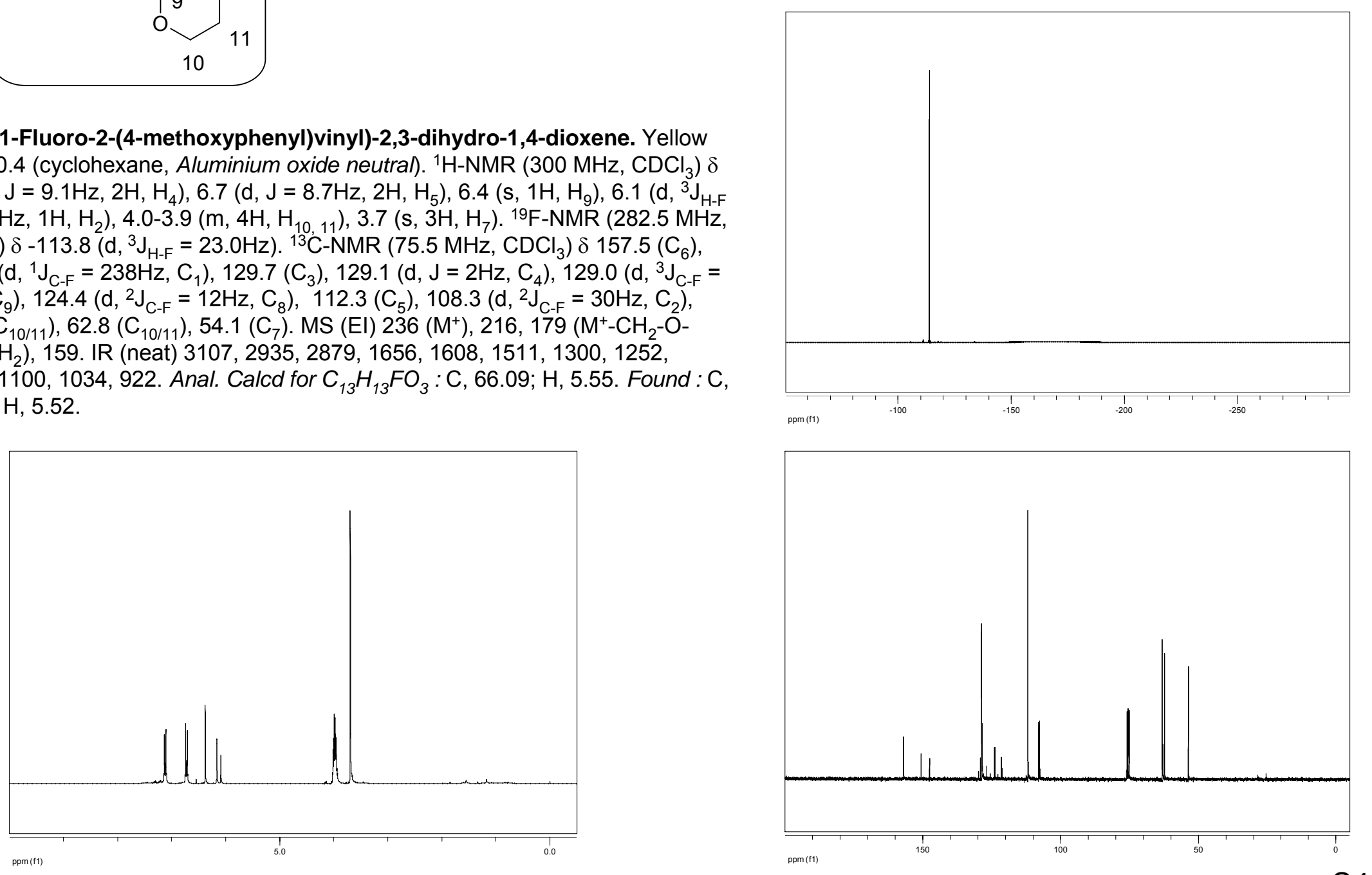


\section{Compound $\mathbf{7}_{\mathbf{2}}(\boldsymbol{E})$}

(E) 5-(1-Fluoro-4-phenylbut-1-enyl)-2,3-dihydro-1,4-dioxene. Colourless oil, $R_{f} 0.3$ (cyclohexane, Aluminium oxide neutral). ${ }^{1} \mathrm{H}-\mathrm{NMR}\left(300 \mathrm{MHz}, \mathrm{CDCl}_{3}\right) \delta 7.2-$ $7.1\left(\mathrm{~m}, 5 \mathrm{H}, \mathrm{H}_{6,7,8}\right), 6.2\left(\mathrm{~s}, 1 \mathrm{H}, \mathrm{H}_{10}\right), 5.1\left(\mathrm{dt},{ }^{3} \mathrm{~J}_{\mathrm{H}-\mathrm{F}}=23.7 \mathrm{~Hz},{ }^{3} \mathrm{~J}_{\mathrm{H}-\mathrm{H}}=7.9 \mathrm{~Hz}, 1 \mathrm{H}\right.$, $\left.\mathrm{H}_{2}\right), 4.0$ (bs, $4 \mathrm{H}, \mathrm{H}_{11}$ ) $)$ 2.6-2.4 (m, 4H, $\left.\mathrm{H}_{3} 4\right)$. ${ }^{19} \mathrm{~F}-\mathrm{NMR}\left(282.5 \mathrm{MHz} \mathrm{CDCl}_{3}\right) \delta-$ $121.5\left(\mathrm{~d},{ }^{3} \mathrm{~J}_{\mathrm{H}-\mathrm{F}}=23.8 \mathrm{~Hz}\right) .{ }^{13} \mathrm{C}-N M R\left(75.5 \mathrm{MHz}^{4} \mathrm{CDCl}_{3}\right) \delta 151.3\left(\mathrm{~d},{ }^{1} \mathrm{~J}_{\mathrm{C}-\mathrm{F}}=233 \mathrm{~Hz}\right.$, $\left.\mathrm{C}_{1}\right), 141.9\left(\mathrm{C}_{5}\right), 131.2\left(\mathrm{~d},{ }^{2} \mathrm{~J}_{\mathrm{C}-\mathrm{F}}=37 \mathrm{~Hz}, \mathrm{C}_{9}\right), 128.9\left(\mathrm{C}_{6 / 7}\right), 128.8\left(\mathrm{C}_{6 / 7}\right), 128.7$ $\left(\mathrm{C}_{10}\right), 126.4\left(\mathrm{C}_{8}\right), 108.3\left(\mathrm{~d},{ }^{2} \mathrm{~J}_{\mathrm{C}-\mathrm{F}}=23 \mathrm{~Hz}, \mathrm{C}_{2}\right), 64.9\left(\mathrm{C}_{11 / 12}\right), 64.4\left(\mathrm{C}_{11 / 12}\right), 36.9(\mathrm{~d}$ $\left.{ }^{4} \mathrm{~J}_{\mathrm{C}-\mathrm{F}}=2 \mathrm{~Hz}, \mathrm{C}_{4}\right), 27.1\left(\mathrm{~d},{ }^{3} \mathrm{~J}_{\mathrm{C}-\mathrm{F}}=8 \mathrm{~Hz}, \mathrm{C}_{3}\right) . \mathrm{MS}(\mathrm{El}) 234\left(\mathrm{M}^{+}\right), 214,157\left(\mathrm{M}^{+}-\mathrm{Ph}\right)$, $143\left(\mathrm{M}^{+}-\mathrm{CH}_{2} \mathrm{Ph}\right), 99,91\left(\mathrm{PhCH}_{2}^{+}\right)$. IR (neat) 3026, 2932, 2879, 1679, 1632, 1455, 1312, 1164, 1110, 924, 700. Anal. Calcd for $\mathrm{C}_{14} \mathrm{H}_{15} \mathrm{FO}_{2}: \mathrm{C}, 71.78 ; \mathrm{H}, 6.45$. Found: C, 71.88; H, 6.41.



\section{Compound $\mathbf{4} \mathbf{d}_{\mathbf{1}}(\boldsymbol{E})$}

(E) 3-Fluoro-1-hydroxy-4(4-methoxyphenyl)but-3-en-2-one. Yellow oil, $R_{f} 0.4$ (33\% AcOEt in cyclohexane). ${ }^{1} \mathrm{H}-\mathrm{NMR}\left(300 \mathrm{MHz}, \mathrm{CDCl}_{3}\right) \delta 7.8(\mathrm{~d}, \mathrm{~J}=8.7 \mathrm{~Hz}, 2 \mathrm{H}$, $\left.\mathrm{H}_{4}\right), 6.9\left(\mathrm{~d}, \mathrm{~J}=9.0 \mathrm{~Hz}, 2 \mathrm{H}, \mathrm{H}_{5}\right), 6.8\left(\mathrm{~d},{ }^{3} \mathrm{~J}_{\mathrm{H}-\mathrm{F}}=26.9 \mathrm{~Hz}, 1 \mathrm{H}, \mathrm{H}_{2}\right), 4.5\left(\mathrm{~m}, 2 \mathrm{H}, \mathrm{H}_{9}\right), 3.9$ $\left(\mathrm{s}, 3 \mathrm{H}, \mathrm{H}_{7}\right), 3.2(\mathrm{~m}, 1 \mathrm{H}, \mathrm{OH}) .{ }^{19} \mathrm{~F}-\mathrm{NMR}\left(282.5 \mathrm{MHz}, \mathrm{CDCl}_{3}\right) \delta-128.6\left(\mathrm{~d},{ }^{3} \mathrm{~J}_{\mathrm{H}-\mathrm{F}}=\right.$ 26.9Hz). ${ }^{13} \mathrm{C}-N M R\left(75.5 \mathrm{MHz}, \mathrm{CDCl}_{3}\right) \delta 193.6\left(\mathrm{~d},{ }^{2} \mathrm{~J}_{\mathrm{CF}}=41 \mathrm{~Hz}, \mathrm{C}_{8}\right), 161.4\left(\mathrm{C}_{6}\right)$, $150.5\left(\mathrm{~d},{ }^{1} \mathrm{~J}_{\mathrm{C}-\mathrm{F}}=244 \mathrm{~Hz}, \mathrm{C}_{1}\right), 133.4\left(\mathrm{~d}, \mathrm{~J}=3 \mathrm{~Hz} \mathrm{C}_{4}\right), 123.9\left(\mathrm{~d},{ }^{2} \mathrm{~J}_{\mathrm{C}-\mathrm{F}}=28 \mathrm{~Hz}, \mathrm{C}_{2}\right)$, $122.4\left(\mathrm{~d}, \mathrm{~J}=10 \mathrm{~Hz}, \mathrm{C}_{3}\right), 114.0\left(\mathrm{C}_{5}\right), 67.0\left(\mathrm{~d}^{3}{ }^{3} \mathrm{~J}=3 \mathrm{~Hz}, \mathrm{C}_{9}\right), 55.5\left(\mathrm{C}_{7}\right) . \mathrm{MS}(\mathrm{EI})$ $210\left(\mathrm{M}^{+}\right), 179\left(\mathrm{M}^{+}-\mathrm{OCH}_{3}\right), 161\left(\mathrm{M}^{+}-\mathrm{OCH}_{3} \& \mathrm{H}_{2} \mathrm{O}\right)$. IR (neat) 3438, 3392, 3100 , 2964, 2840, 1713, 1644, 1602, 1514, 1301, 1259, 1172, 1097, 1030, 831. Anal. Calcd for $\mathrm{C}_{11} \mathrm{H}_{11} \mathrm{FO}_{3}$ : C, 62.85; H, 5.27. Found: C, 62.74; H, 5.36.
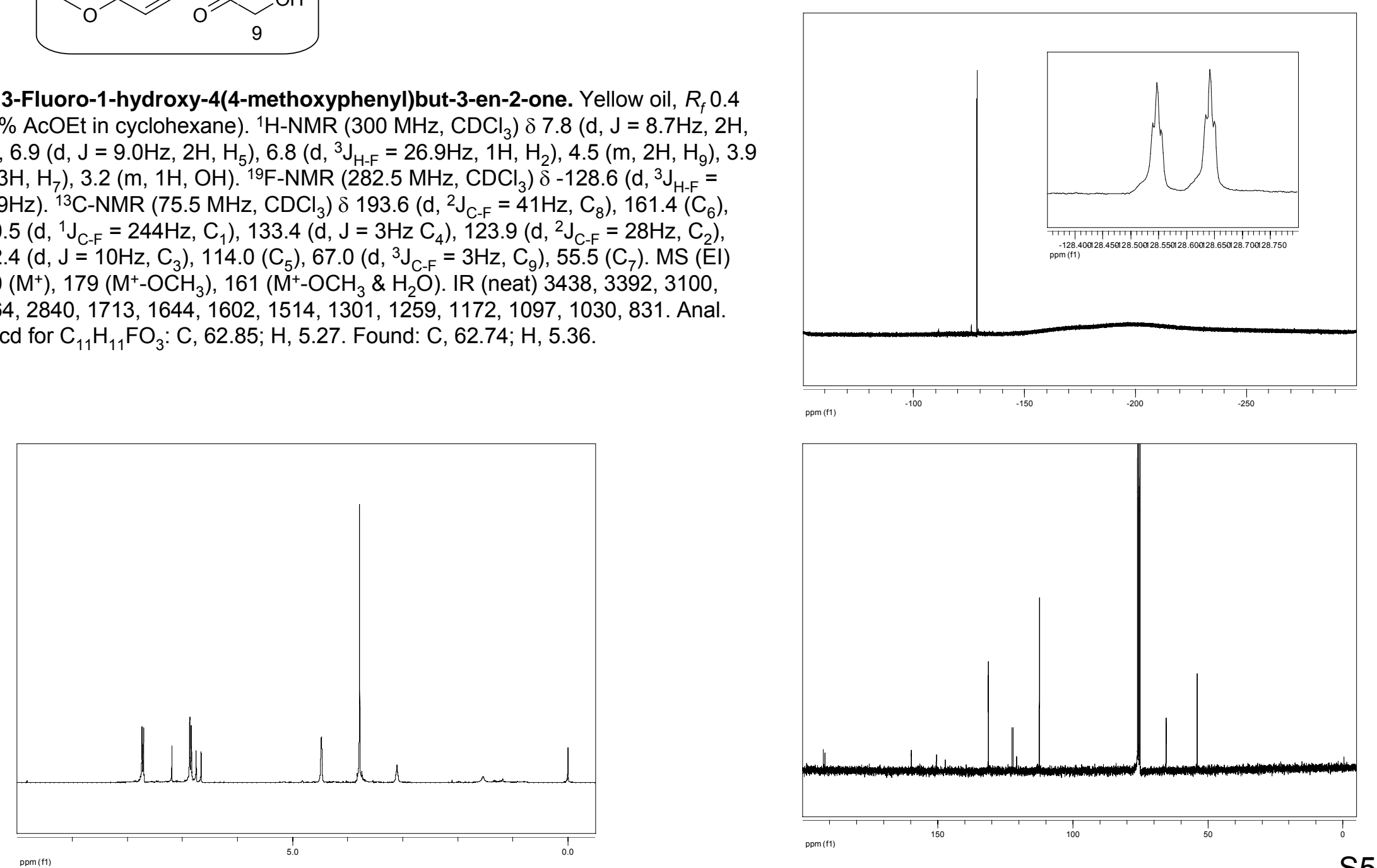


\section{Compound $10(R, Z)$}

$(Z)$

(R)-3-Fluoro-5-methyl-6-(oxytert-butyl

diphenylsilyl)-3-en-2-one. Colourless oil, Rf $0.56\left(20 \%\right.$ AcOEt in cyclohexane), $[\alpha]_{20} \mathrm{D}=-5^{\circ}\left(\mathrm{c}=0.1, \mathrm{CH}_{2} \mathrm{Cl}_{2}\right)$. ${ }^{1} \mathrm{H}-N M R\left(300 \mathrm{MHz}, \mathrm{CDCl}_{3}\right)$ ठ 7.7-7.6 (m, 4H, $\left.\mathrm{H}_{9}\right), 7.4-7.3\left(\mathrm{~m}, 6 \mathrm{H}, \mathrm{H}_{10},{ }_{11}\right), 5.9-6.0$ $\left(\mathrm{dd},{ }^{3} \mathrm{~J}_{\mathrm{H}-\mathrm{F}}=34.5 \mathrm{~Hz},{ }^{3} \mathrm{~J}_{\mathrm{H}-\mathrm{H}}=9.6 \mathrm{~Hz}, 1 \mathrm{H}, \mathrm{H}_{2}\right), 3.6-3.5\left(\mathrm{~m}, 2 \mathrm{H}, \mathrm{H}_{5}\right), 3.0-2.9(\mathrm{~m}, 1 \mathrm{H}$, $\left.\mathrm{H}_{3}\right), 2.3\left(\mathrm{~d},{ }^{4} \mathrm{~J}_{\mathrm{H}-\mathrm{F}}=2.8 \mathrm{~Hz}, 3 \mathrm{H}, \mathrm{H}_{13}\right), 1.1\left(\mathrm{~d},{ }^{3} \mathrm{~J}_{\mathrm{H}-\mathrm{H}}=6.9 \mathrm{~Hz}, 3 \mathrm{H}, \mathrm{H}_{4}\right), 1.0\left(\mathrm{~s}, 9 \mathrm{H}, \mathrm{H}_{7}\right)$. ${ }^{19} \mathrm{~F}-\mathrm{NMR}\left(282.5 \mathrm{MHz}, \mathrm{CDCl}_{3}\right) \delta-127.9$ (s). ${ }^{13} \mathrm{C}-\mathrm{NMR}\left(75.5 \mathrm{MHz}, \mathrm{CDCl}_{3}\right) \delta 191.9$ $\left(\mathrm{d},{ }^{2} \mathrm{~J}_{\mathrm{C}-\mathrm{F}}=31.9 \mathrm{~Hz}, \mathrm{C}_{12}\right), 155.2\left(\mathrm{~d},{ }^{1} \mathrm{~J}_{\mathrm{C}-\mathrm{F}}=260.9 \mathrm{~Hz}, \mathrm{C}_{1}\right), 135.9\left(\mathrm{C}_{9}\right), 133.8\left(\mathrm{C}_{8}\right)$, $130.1\left(\mathrm{C}_{11}\right), 128.1\left(\mathrm{C}_{10}\right), 122.9\left(\mathrm{~d},{ }^{2} \mathrm{~J}_{\mathrm{C}-\mathrm{F}}=12.4 \mathrm{~Hz}, \mathrm{C}_{2}\right), 67.8\left(\mathrm{C}_{5}\right), 33.1\left(\mathrm{C}_{3}\right), 27.0$ $\left(\mathrm{d},{ }^{3} \mathrm{~J}_{\mathrm{C}-\mathrm{F}}=10.9 \mathrm{~Hz}, \mathrm{C}_{13}\right), 25.9\left(\mathrm{C}_{7}\right), 19.7\left(\mathrm{C}_{6}\right), 16.7\left(\mathrm{C}_{4}\right) . \mathrm{MS}(\mathrm{El}) 327(\mathrm{M}+-\mathrm{tBu})$ 201, 181, 57. IR (neat) $3073,2968,2926,2863,2357,1768,1484,1368,1115$ $\mathrm{cm}^{-1}$. Anal. Calcd for $\mathrm{C}_{23} \mathrm{H}_{29} \mathrm{FO}_{2} \mathrm{Si}: \mathrm{C}, 71.83 ; \mathrm{H}, 7.60$. Found : C, 71.25; $\mathrm{H}, 7.32$.
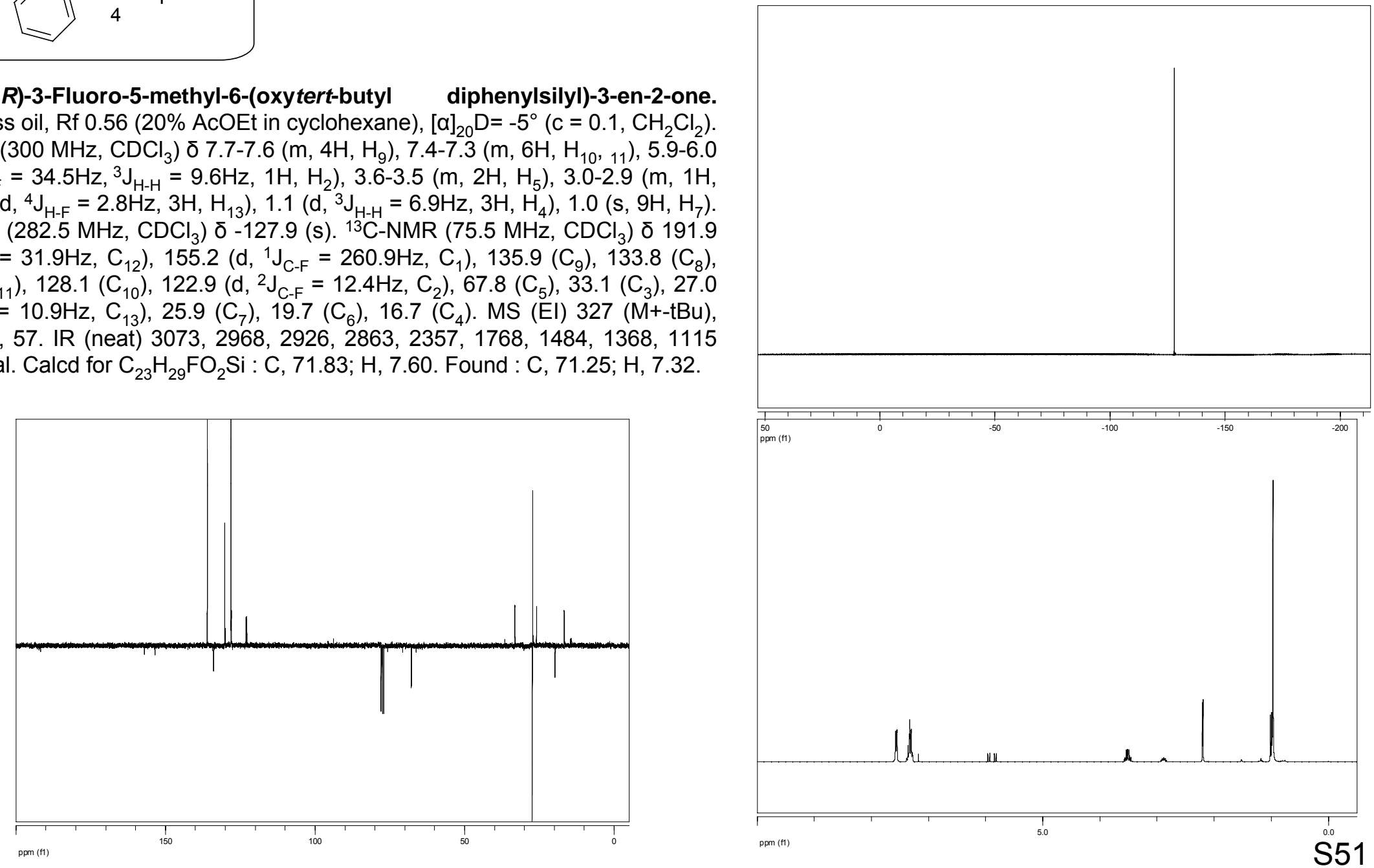


\section{Compound $10(R, E)$}

(E) (R)-3-Fluoro-5-methyl-6-(oxytert-butyl diphenylsilyl)hex-3-en-2-one Yellow oil, $\mathrm{Rf}=0.69(20 \%$ AcOEt in cyclohexane $),[\alpha]^{20} \mathrm{D}=-40^{\circ},(\mathrm{c}=0.057$, $\left.\mathrm{CH}_{2} \mathrm{Cl}_{2}\right) .{ }^{1} \mathrm{H}-\mathrm{NMR}\left(300 \mathrm{MHz}, \mathrm{CDCl}_{3}\right)$ ठ 7.7-7.6 (m, 4H, $\left.\mathrm{H}_{9}\right), 7.4-7.3\left(\mathrm{~m}, 6 \mathrm{H}, \mathrm{H}_{10}\right.$, 11), 5.7-5.6 (dd, $\left.{ }^{3} \mathrm{~J}_{\mathrm{H}-\mathrm{F}}=22.6 \mathrm{~Hz},{ }^{3} \mathrm{~J}_{\mathrm{H}-\mathrm{H}}=9.8 \mathrm{~Hz}, 1 \mathrm{H}, \mathrm{H}_{2}\right), 3.6-3.5\left(\mathrm{~m}, 3 \mathrm{H}, \mathrm{H}_{56}\right)$ $2.3\left(\mathrm{~d},{ }^{5} \mathrm{~J}_{\mathrm{H}-\mathrm{F}}=4.9 \mathrm{~Hz}, 1 \mathrm{H}, \mathrm{H}_{13}\right), 1.1\left(\mathrm{~d},{ }^{3} \mathrm{~J}_{\mathrm{H}-\mathrm{H}}=2.1 \mathrm{~Hz}, 3 \mathrm{H}, \mathrm{H}_{4}\right), 1.0\left(\mathrm{~s}, 9 \mathrm{H}, \mathrm{H}_{7}\right)$. ${ }^{19} \mathrm{~F}-\mathrm{NMR}\left(282.5 \mathrm{MHz}, \mathrm{CDCl}_{3}\right) \delta-119.0 .{ }^{13} \mathrm{C}-\mathrm{NMR}\left(75.5 \mathrm{MHz}, \mathrm{CDCl}_{3}\right) \delta 194.5$ $\left(\mathrm{d},{ }^{2} \mathrm{~J}_{\mathrm{C}-\mathrm{F}}=40.0 \mathrm{~Hz}, \mathrm{C}_{1}\right), 153.4\left(\mathrm{~d},{ }^{1} \mathrm{~J}_{\mathrm{C}-\mathrm{F}}=255.9 \mathrm{~Hz}, \mathrm{C}_{2}\right), 135.8\left(\mathrm{C}_{10}\right), 133.7\left(\mathrm{C}_{8}\right)$, $129.8\left(\mathrm{C}_{11}\right), 127.8\left(\mathrm{C}_{9}\right), 124.3\left(\mathrm{~d},{ }^{2} \mathrm{~J}_{\mathrm{C}-\mathrm{F}}=17.4 \mathrm{~Hz}, \mathrm{C}_{3}\right), 68.1\left(\mathrm{C}_{5}\right), 32.8\left(\mathrm{~d},{ }^{3} \mathrm{~J}_{\mathrm{C}-\mathrm{F}}\right.$ $\left.=5.1 \mathrm{~Hz}, \mathrm{C}_{4}\right), 27.9\left(\mathrm{~d},{ }^{3} \mathrm{~J}_{\mathrm{C}-\mathrm{F}}=2.9 \mathrm{~Hz}, \mathrm{C}_{13}\right), 26.9\left(\mathrm{C}_{7}\right), 19.4\left(\mathrm{C}_{6}\right), 17.0\left(\mathrm{~d},{ }^{4} \mathrm{~J}_{\mathrm{C}-\mathrm{F}}=\right.$ $2.2 \mathrm{~Hz}, \mathrm{C}_{12}$ ). MS (El) 327 (M+-tBu), 201, 199, 181, 141, 57. IR (neat) 2959, 2856, 1708, 1427, 1363, 1195, 1112, 823, $741 \mathrm{~cm}^{-1}$. Anal. Calcd for $\mathrm{C}_{23} \mathrm{H}_{29} \mathrm{FO}_{2} \mathrm{Si}: \mathrm{C}, 71.83 ; \mathrm{H}, 7.60$. Found: $\mathrm{C}, 71.15 ; \mathrm{H}, 7.49$.

Determination of the diastereoisomeric purities of imines derived from $10(R, Z)$ and $10(R, E) ;{ }^{19} F$ NMR spectra
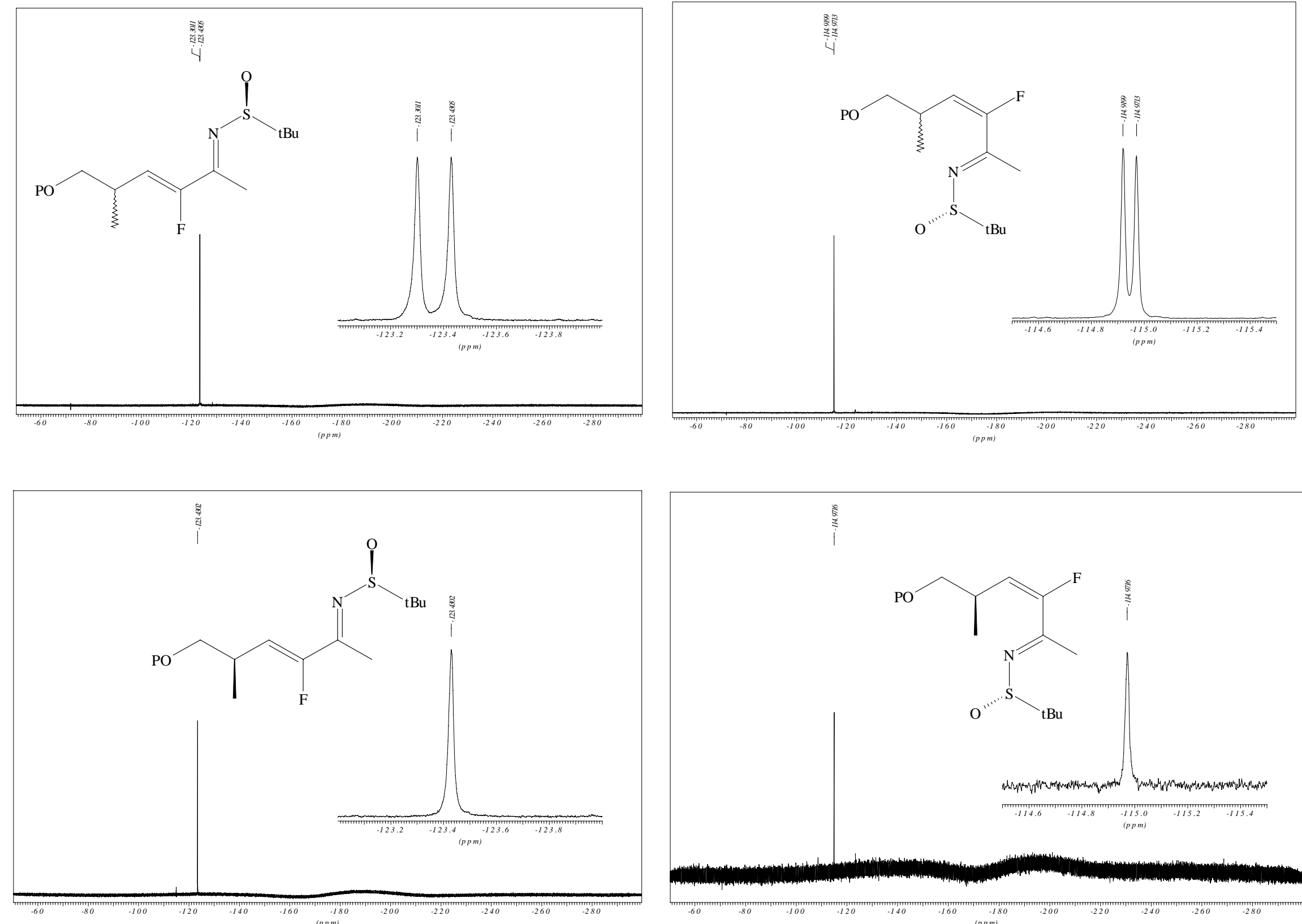